NBER WORKING PAPER SERIES

\title{
INFLATION BETS OR DEFLATION HEDGES? THE CHANGING RISKS OF NOMINAL BONDS
}

\author{
John Y. Campbell \\ Adi Sunderam \\ Luis M. Viceira
}

Working Paper 14701

http://www.nber.org/papers/w14701

\author{
NATIONAL BUREAU OF ECONOMIC RESEARCH \\ 1050 Massachusetts Avenue \\ Cambridge, MA 02138 \\ February 2009
}

We are grateful to Geert Bekaert, Jesus Fernandez-Villaverde, Wayne Ferson, Javier Gil-Bazo, Pablo Guerron, John Heaton, Ravi Jagannathan, Jon Lewellen, Monika Piazzesi, Pedro Santa-Clara, George Tauchen, and seminar participants at the 2009 Annual Meeting of the American Finance Association, Bank of England, European Group of Risk and Insurance Economists 2008 Meeting, Harvard Business School Finance Unit Research Retreat, Imperial College, Marshall School of Business, NBER Fall 2008 Asset Pricing Meeting, Norges Bank, Society for Economic Dynamics 2008 Meeting, Stockholm School of Economics, Tilburg University, Tuck Business School, and Universidad Carlos III in Madrid for hepful comments and suggestions. This material is based upon work supported by the National Science Foundation under Grant No. 0214061 to Campbell, and by Harvard Business School Research Funding. The views expressed herein are those of the author(s) and do not necessarily reflect the views of the National Bureau of Economic Research.

NBER working papers are circulated for discussion and comment purposes. They have not been peerreviewed or been subject to the review by the NBER Board of Directors that accompanies official NBER publications.

(C) 2009 by John Y. Campbell, Adi Sunderam, and Luis M. Viceira. All rights reserved. Short sections of text, not to exceed two paragraphs, may be quoted without explicit permission provided that full credit, including $\odot$ notice, is given to the source. 
Inflation Bets or Deflation Hedges? The Changing Risks of Nominal Bonds

John Y. Campbell, Adi Sunderam, and Luis M. Viceira

NBER Working Paper No. 14701

February 2009, Revised July 2013

JEL No. G0,G10,G11,G12

\begin{abstract} by four state variables: the real interest rate, temporary and permanent components of expected inflation, and the "nominal-real covariance" of inflation and the real interest rate with the real determined by the nominal-real covariance. The concavity of the yield curve -- the level of intermediate-term bond yields, relative to the average of short- and long-term bond yields -- is a good driving down term premia.

John Y. Campbell

Morton L. and Carole S.

Olshan Professor of Economics

Department of Economics

Harvard University

Littauer Center 213

Cambridge, MA 02138

and NBER

john_campbell@harvard.edu

Adi Sunderam

420M Baker Library

Harvard Business School

Boston, MA 02163

sunderam@fas.harvard.edu

Luis M. Viceira

George E. Bates Professor

Harvard Business School

Baker Library 367

Boston, MA 02163

and NBER

lviceira@hbs.edu
\end{abstract}

The covariance between US Treasury bond returns and stock returns has moved considerably over time. While it was slightly positive on average in the period 1953-2009, it was unusually high in the early 1980's and negative in the early 2000's, partucularly in the downturns of 2000-02 and 2007-09. This paper specifies and estimates a model in which the nominal term structure of interest rates is driven economy. The last of these state variables enables the model to fit the changing covariance of bond and stock returns. Log bond yields and term premia are quadratic in these state variables, with term premia proxy for the level of term premia. The nominal-real covariance has declined since the early 1980's, 


\section{Introduction}

In recent years investors have come to regard US Treasury bonds as hedges, assets that perform well when other assets lose value, and more generally when bad macroeconomic news arrives. During both of the two most recent stock market and macroeconomic downturns, in 2000-02 and 2007-09, Treasury bonds performed well. In addition, for the past decade and particularly during these downturns, Treasury bond returns have been negatively correlated with stock returns at a daily frequency. In previous decades, however, Treasury bonds performed very differently; they were either uncorrelated or positively correlated with stock returns. The purpose of this paper is to highlight these changes in magnitude and switches in sign of the covariation between bonds and stocks, and to ask what they imply for bond risk premia and the shape of the term structure of interest rates.

To understand how a changing bond-stock covariance can affect the pricing of Treasury bonds, we specify and estimate a multifactor term structure model that incorporates traditional macroeconomic influences - real interest rates and expected inflation - along with a state variable driving the variance of real and nominal interest rates and their covariance with the macroeconomy. The model is set up so that all factors have an economic interpretation, and the covariance of bond returns with the macroeconomy can switch sign. For simplicity, the basic version of the model assumes a constant price of risk, or equivalently, a constant variance for the stochastic discount factor. We estimate the model using postwar quarterly US time series for nominal and inflation-indexed bond yields, stock returns, realized and forecast inflation, and the realized second moments of bond and stock returns calculated from daily data within each quarter. The use of realized second moments, unusual in the term structure literature, forces our model to fit the historically observed changes in risks.

Our model delivers three main results. First, the risk premia of nominal Treasury bonds should have changed over the decades because of changes in the covariance between inflation and the real economy. The model predicts positive nominal bond risk premia in the early 1980s, when bonds covaried positively with stocks, and negative risk premia in the 2000s and particularly during the downturn of 2007-09, when bonds hedged equity risk.

Second, a strongly concave term structure of interest rates, with high interest rates at a maturity around 3 years relative to short- and long-term interest rates, should predict high excess bond returns. In the model, a high bond-stock covariance 
is associated with a high volatility of bond returns. The high bond-stock covariance generates a high term premium and a steep yield curve at maturities of 1-3 years, while the high bond volatility lowers long-term yields through a Jensen's inequality or convexity effect. Thus, the concavity of the yield curve is a good proxy for the bond-stock covariance. In this fashion, our model explains the qualitative finding of Cochrane and Piazzesi (2005) that a tent-shaped linear combination of forward rates, with a peak at about 3 years, predicts excess bond returns at all maturities.

Third, however, our model does not explain the volatility of term premia implied by predictive regressions of excess bond returns onto bond yields. Whether these regressions use maturity-matched yield spreads (Campbell and Shiller 1991), maturity-matched forward spreads (Fama and Bliss 1987), or a multi-maturity combination of forward rates (Cochrane and Piazzesi 2005), they imply much greater variability of expected excess bond returns than is captured by our model. This negative finding implies that bond risk premia respond to other factors besides the bond-stock covariance. It is an open question whether these factors are best modeled using an exogenously changing price of risk, as in the literature on essentially affine bond pricing models following Duffee (2002), or whether other variables such as the supply of Treasury bonds need to be incorporated into the analysis as advocated by Greenwood and Vayanos (2012) and Krishnamurthy and Vissing-Jorgensen (2012).

To illustrate the basic observation that motivates this paper, Figure 1 plots the history of the realized covariance of 10-year nominal zero-coupon Treasury bonds with the CRSP value-weighted stock index, calculated using a rolling three-month window of daily data. For ease of interpretation, the figure also shows the history of the realized beta of Treasury bonds with stocks (the bond-stock covariance divided by the realized variance of stock returns), as this allows a simple back-of-the-envelope calculation of the term premium that would be implied by the simple Capital Asset Pricing Model (CAPM) given any value for the equity premium. The covariance (plotted on the left vertical scale) and beta (on the right vertical scale) move closely together, with the major divergences occurring during periods of low stock return volatility in the late 1960s and the mid-1990s.

Figure 1 displays a great deal of high-frequency variation in both series, much of which is attributable to noise in realized second moments. But it also shows substantial low-frequency movements. The beta of bonds with stocks was close to zero in the mid-1960's and mid-1970's, much higher with an average around 0.4 in the 1980's, spiked in the mid-1990's, and declined to negative average values in the 
2000's. During the two downturns of 2000-02 and 2007-09, the average realized beta of Treasury bonds was about -0.2. Thus from peak to trough, the realized beta of Treasury bonds has declined by about 0.6 and has changed its sign. According to the CAPM, this would imply that term premia on 10-year zero-coupon Treasuries should have declined by $60 \%$ of the equity premium.

Nominal bond returns respond both to expected inflation and to real interest rates. A natural question is whether the pattern shown in Figure 1 reflects a changing covariance of inflation with the stock market, or a changing covariance of real interest rates with the stock market. Figure 2 plots the covariance and beta of inflation shocks with stock returns, using a rolling three-year window of quarterly data and a firstorder quarterly vector autoregression for inflation, stock returns, and the three-month Treasury bill yield to calculate inflation shocks. Because high inflation is associated with high bond yields and low bond returns, the figure shows the covariance and beta for realized deflation shocks (the negative of inflation shocks) which should move in the same manner as the bond return covariance and beta reported in Figure 1. Indeed, Figure 2 shows a similar history for the deflation covariance as for the nominal bond covariance.

Real interest rates also play a role in changing nominal bond risks. In the period since 1997, when long-term Treasury inflation-protected securities (TIPS) were first issued, Campbell, Shiller, and Viceira (2009) report that TIPS have had a predominantly negative beta with stocks. Like the nominal bond beta, the TIPS beta was particularly negative in the downturns of 2000-02 and 2007-09. Thus not only the stock-market covariances of nominal bond returns, but also the covariances of two proximate drivers of those returns, inflation and real interest rates, change over time and occasionally switch sign. We design our term structure model to fit these facts.

The organization of the paper is as follows. Section 2 briefly reviews the related literature. Section 3 presents our model of the real and nominal term structures of interest rates. Section 4 describes our estimation method and presents parameter estimates and historical fitted values for the unobservable state variables of the model. Section 5 discusses the implications of the model for the shape of the yield curve and the movements of risk premia on nominal bonds. Section 6 concludes. An Appendix to this paper available online (Campbell, Sunderam, and Viceira 2013) presents details of the model solution and additional empirical results. 


\section{Literature Review}

Despite the striking movements in the bond-stock covariance illustrated in Figure 1, this second moment has received relatively little attention in the enormous literature on the term structure of interest rates. ${ }^{2}$ One reason for this neglect may be that until the last 15 years, the covariance was almost always positive and thus it was not apparent that it could switch sign. In the absence of a sign switch, a model of changing bond market volatility, with a constant correlation or even a constant covariance between bonds and stocks, might be adequate.

The early literature on the term structure of interest rates concentrated on testing the null hypothesis of constant bond risk premia, also known as the expectations hypothesis of the term structure (Shiller, Campbell, and Schoenholtz 1983, Fama and Bliss 1987, Stambaugh 1988, Campbell and Shiller 1991). Second-generation affine term structure models such as Cox, Ingersoll, and Ross (1985) modeled changes in bond market volatility linked to the short-term interest rate. This approach encounters the difficulty that bond market volatility appears to move independently of the level of interest rates. In addition, the empirical link between bond market volatility and the expected excess bond return is weak, although some authors such as Campbell (1987) do estimate it to be positive. ${ }^{3}$

In the last ten years a large literature has specified and estimated essentially affine term structure models (Duffee 2002), in which a changing price of risk can affect bond market risk premia without any change in the quantity of risk, while risk premia are linear functions of bond yields (Dai and Singleton 2002, Sangvinatsos and Wachter 2005, Wachter 2006, Buraschi and Jiltsov 2007, Bekaert, Engstrom, and Xing 2009, Bekaert, Engstrom, and Grenadier 2010). Models such as those of Dai and Singleton (2002) and Sangvinatsos and Wachter (2005) achieve a good fit to the historical term structure, but this literature uses latent factors that are hard to interpret economically.

\footnotetext{
${ }^{2}$ Important exceptions in the last decade include Li (2002), Guidolin and Timmermann (2006), Christiansen and Ranaldo (2007), David and Veronesi (2009), Baele, Bekaert, and Inghelbrecht (2010), and Viceira (2012).

${ }^{3}$ More recently, Piazzesi and Schneider (2006) and Rudebusch and Wu (2007) have built affine models of the nominal term structure in which a reduction of inflation uncertainty drives down the risk premia on nominal bonds towards the lower risk premia on inflation-indexed bonds. Similarly, Backus and Wright (2007) argue that declining uncertainty about inflation explains the low yields on nominal Treasury bonds in the mid-2000's.
} 
Some papers have extended the essentially affine approach to model stock and bond prices jointly (Mamaysky 2002, d'Addona and Kind 2006, Bekaert, Engstrom, and Grenadier 2010). Eraker (2008), Hasseltoft (2009), and Bansal and Shaliastovich (2013) price both stocks and bonds using the consumption-based long-run risks model of Bansal and Yaron (2004). However none of these papers allow the bond-stock covariance to change sign.

There is a small empirical literature decomposing nominal bond returns into economically interpretable shocks to real interest rates, inflation expectations, and risk premia, and estimating the covariances of these components with stock returns (Barsky 1989, Shiller and Beltratti 1992, Campbell and Ammer 1993). A weakness of this literature is that the estimated covariances are assumed to be constant over time, an assumption relaxed by Viceira (2012).

In this paper we want to model a time-varying covariance between state variables and the stochastic discount factor, which can switch sign. Duffie and Kan (1996) point out that this can be done within an affine framework if we allow the state variables to be bond yields rather than fundamental macroeconomic variables. In this spirit, Buraschi, Cieslak, and Trojani (2008) expand the state space of a nonlinear model to obtain an affine model in which correlations can switch sign. The cost of this approach is that the factors in the model become difficult to interpret. Instead, we use interpretable macroeconomic variables as factors and write a linear-quadratic model like those of Beaglehole and Tenney (1991), Constantinides (1992), Ahn, Dittmar and Gallant (2002), and Realdon (2006).

To solve our model, we use a general result on the expected value of the exponential of a non-central chi-squared distribution which we take from the Appendix to Campbell, Chan, and Viceira (2003). To estimate the model, we use a nonlinear filtering technique, the unscented Kalman filter, proposed by Julier and Uhlmann (1997), reviewed by Wan and van der Merwe (2001), and recently applied in finance by Binsbergen and Koijen (2008). 


\section{$3 \quad$ A Quadratic Bond Pricing Model}

We now present a term structure model that allows for time variation in the covariances between real interest rates, inflation, and the real economy. In the model, both real and nominal bond yields are linear-quadratic functions of the vector of state variables and, consistent with the empirical evidence, the conditional volatilities and covariances of excess returns on real and nominal assets are time varying.

Before describing the model, it is worth discussing our motivation for writing down a quadratic model, rather than an essentially affine model as is more common in the literature. A key goal of the paper is understand whether the variations in the quantity of bond risk documented in Figure 1 reflect variation in the quantity of real interest rate risk, the quantity of inflation risk, or a combination of both. This motivates us to build a model where the state variables are explicitly identified with economic quantities. This motivation, in combination with the fact that we allow for time-varying variances and covariances, means that we must venture outside the class of essentially affine models.

\subsection{The SDF and the real term structure}

We start by assuming that the log of the real stochastic discount factor (SDF), $m_{t+1}=$ $\log \left(M_{t+1}\right)$, follows the process:

$$
-m_{t+1}=x_{t}+\frac{\sigma_{m}^{2}}{2}+\varepsilon_{m, t+1}
$$

For simplicity, the SDF innovation $\varepsilon_{m, t+1}$ is homoskedastic although we have developed and estimated an extension of the model with a heteroskedastic SDF. ${ }^{4}$ The drift $x_{t}$, however, follows an $\mathrm{AR}(1)$ process subject to both a heteroskedastic shock $\psi_{t} \varepsilon_{x, t+1}$ and a homoskedastic shock $\varepsilon_{X, t+1}$ :

$$
x_{t+1}=\mu_{x}\left(1-\phi_{x}\right)+\phi_{x} x_{t}+\psi_{t} \varepsilon_{x, t+1}+\varepsilon_{X, t+1} .
$$

\footnotetext{
${ }^{4}$ Details of the more general model are available from the authors upon request. The more general specification captures the spirit of recent term structure models by Bekaert et al (2005), Buraschi and Jiltsov (2007), Wachter (2006) and others in which time-varying risk aversion drives time-varying bond risk premia.
} 
The innovations $\varepsilon_{m, t+1}, \varepsilon_{x, t+1}$, and $\varepsilon_{X, t+1}$ are normally distributed, with zero means and constant variance-covariance matrix. We allow these shocks to be cross-correlated and adopt the notation $\sigma_{i}^{2}$ to describe the variance of shock $\varepsilon_{i}$, and $\sigma_{i j}$ to describe the covariance between shock $\varepsilon_{i}$ and shock $\varepsilon_{j}$. To reduce the complexity of the equations that follow, we assume that the shocks to $x_{t}$ are orthogonal to each other; that is, $\sigma_{x X}=0$.

The state variable $x_{t}$ is the short-term log real interest rate. The price of a single-period zero-coupon real bond satisfies $P_{1, t}=\mathrm{E}_{t}\left[\exp \left\{m_{t+1}\right\}\right]$, so that its yield $y_{1 t}=-\log \left(P_{1, t}\right)$ equals

$$
y_{1 t}=-\mathrm{E}_{t}\left[m_{t+1}\right]-\frac{1}{2} \operatorname{Var}_{t}\left(m_{t+1}\right)=x_{t}
$$

The model has an additional state variable, $\psi_{t}$, which governs time variation in the volatility of the real interest rate and its covariation with the SDF. ${ }^{5}$ We assume that $\psi_{t}$ follows a standard homoskedastic $\mathrm{AR}(1)$ process:

$$
\psi_{t+1}=\mu_{\psi}\left(1-\phi_{\psi}\right)+\phi_{\psi} \psi_{t}+\varepsilon_{\psi, t+1} \text {. }
$$

Importantly, this process can change sign, so the covariance of the real interest rate with the SDF and the price of real interest rate risk can be either positive or negative. Because the model is observationally equivalent when both $\psi_{t}$ and the shocks it multiplies switch sign, without loss of generality we normalize the model such that $\psi_{t}$ has a positive mean.

We allow for two shocks in the real interest rate because a single shock would imply a constant Sharpe ratio for real bonds. With only a heteroskedastic shock, the model would also imply that the conditional volatility of the real interest rate would be proportional to the covariance between the real interest rate and the real SDF; equivalently, the conditional correlation of the real rate and the SDF would be constant in absolute value with occasional sign switches. Our specification avoids these implausible implications while remaining reasonably parsimonious.

In this model, the log prices of real bonds are linear in $x_{t}$ and quadratic in $\psi_{t}$ :

$$
p_{n, t}=A_{n}+B_{x, n} x_{t}+B_{\psi, n} \psi_{t}+C_{\psi, n} \psi_{t}^{2},
$$

\footnotetext{
${ }^{5}$ In an earlier version of this paper we assumed a homoskedastic process for the real interest rate, writing a model in which $\psi_{t}$ only affects inflation and nominal interest rates. This generates a simpler affine real term structure of interest rates, but is inconsistent with time-variation in the covariance between TIPS returns and the real economy documented by Campbell, Shiller, and Viceira (2009).
} 
where the coefficients $A_{n}, B_{x, n}, B_{\psi, n}$, and $C_{\psi, n}$ solve a set of recursive equations given in the Appendix. These coefficients are functions of the maturity of the bond $(n)$ and the coefficients that determine the stochastic processes for the state variables. From equation (3), $B_{x, 1}=-1$ and the remaining coefficients are zero at $n=1$.

The conditional risk premium on an $n$-period real bond is linear in $\psi_{t}$ :

$$
\mathrm{E}_{t}\left[r_{n, t+1}-r_{1, t+1}\right]+\frac{1}{2} \operatorname{Var}_{t}\left(r_{n, t+1}-r_{1, t+1}\right)=-\left(A_{n}^{*}+B_{n}^{*} \psi_{t}\right)
$$

where $A_{n}^{*}$ and $B_{n}^{*}$ are functions of $A_{n}, B_{x, n}, B_{\psi, n}$, and $C_{\psi, n}$. In the case of a 2-period real bond, we have $A_{2}^{*}=\sigma_{X m}$ and $B_{2}^{*}=\sigma_{x m}$. To gain intuition about the 2-period real bond risk premium, consider the simple case where $\sigma_{X m}=0$ and $\sigma_{x m} \psi_{t}>0$. This implies that real bond risk premia are negative. The reason for this is that with positive $\sigma_{x m} \psi_{t}$, the real interest rate tends to rise in good times and fall in bad times. Since real bond returns move opposite the real interest rate, real bonds are countercyclical assets that hedge against economic downturns and command a negative risk premium.

\subsection{Inflation and the nominal term structure}

To price nominal bonds, we need a model for inflation. We assume that log inflation $\pi_{t}=\log \left(\Pi_{t}\right)$ follows a linear-quadratic conditionally heteroskedastic process:

$$
\pi_{t+1}=\lambda_{t}+\xi_{t}+\frac{\sigma_{\pi}^{2}}{2} \psi_{t}^{2}+\psi_{t} \varepsilon_{\pi, t+1}
$$

where $\psi_{t}$ is given in (4) and expected log inflation is the sum of two components, a permanent component $\lambda_{t}$ and a transitory component $\xi_{t}$.

The dynamics of these components are given by

$$
\lambda_{t+1}=\lambda_{t}+\varepsilon_{\Lambda, t+1}+\psi_{t} \varepsilon_{\lambda, t+1}
$$

and

$$
\xi_{t+1}=\phi_{\xi} \xi_{t}+\psi_{t} \varepsilon_{\xi, t+1} .
$$

The presence of an integrated component in expected inflation removes the need to include a nonzero mean in the stationary component of expected inflation. 
We assume that the underlying shocks to realized inflation, the components of expected inflation, and conditional inflation volatility $-\varepsilon_{\pi, t+1}, \varepsilon_{\lambda, t+1}, \varepsilon_{\Lambda, t+1}, \varepsilon_{\xi, t+1}$, and $\varepsilon_{\psi, t+1}$ - are again jointly normally distributed zero-mean shocks with a constant variance-covariance matrix. We allow these shocks to be cross-correlated with the shocks to $m_{t+1}$ and $x_{t+1}$. Since $\psi_{t}$ premultiplies all inflation shocks, without loss of generality we set $\sigma_{\pi}$ to an arbitrary value of 1 .

Our inclusion of two components of expected inflation gives our model the flexibility it needs to fit both persistent variation in long-term nominal interest rates and inflation, and transitory variation in short rates relative to long rates. The former requires persistent variation in expected inflation, while the latter requires transitory variation in some state variable. The persistence and volatility of the long-term inflation-indexed bond yield implies that the real interest rate is highly persistent, so under our assumption that a single $\mathrm{AR}(1)$ process drives the real interest rate, we need a transitory component of expected inflation to generate changes in the slope of the nominal yield curve. ${ }^{6}$

We use the same state variable $\psi_{t}$ that drives changing volatility in the real term structure to drive changes in inflation volatility. This keeps our model parsimonious while capturing the inflation heteroskedasticity first modelled by Engle (1982) in a manner consistent with the common movements of nominal and inflation-indexed bond volatility documented by Campbell, Shiller, and Viceira (2009). ${ }^{7}$

We allow both a homoskedastic shock $\varepsilon_{\Lambda, t+1}$ and a heteroskedastic shock $\psi_{t} \varepsilon_{\lambda, t+1}$ to impact the permanent component of expected inflation. The reasons for this assumption are similar to those that lead us to assume two shocks for the real interest rate process. In the absence of a homoskedastic shock to expected inflation, the conditional volatility of expected inflation would be proportional to the conditional

\footnotetext{
${ }^{6}$ There are other specifications that could be used to fit these facts. We impose a unit root on the persistent component of expected inflation for convenience of model analysis and estimation, but a near-unit root would also be viable. Regime-switching models offer an alternative way to reconcile persistent fluctuations with stationary long-run behavior of interest rates (Garcia and Perron 1996, Gray 1996, Bansal and Zhou 2002, Ang, Bekaert, and Wei 2008). We could also allow the real interest rate to have both a persistent and transitory component, in which case expected inflation could be purely persistent. Our specification is consistent with Cogley, Primiceri, and Sargent (2010) and generalizes Stock and Watson (2007) to allow some persistence in the stationary component of inflation. Mishkin (1990) presents evidence that bond yield spreads forecast future changes in inflation, which is also consistent with our specification.

${ }^{7}$ Although not reported in the article, the correlation in their data between the volatility of nominal US Treasury bond returns and the volatility of TIPS returns is slightly greater than 0.7 .
} 
covariance between expected inflation and real economic variables. There is no economic reason to expect that these two second moments should be proportional to one another, and the data suggest that the conditional covariance can be close to zero even when the conditional volatility remains positive. Put another way, the presence of two shocks allows the conditional correlation between real and nominal variables to vary smoothly rather than being fixed in absolute value with occasional sign switches. Since long-term expected inflation is the main determinant of longterm nominal interest rates, we allow two shocks to this process but for parsimony allow only heteroskedastic shocks to transitory expected and realized inflation.

The process for realized inflation, equation (7), is formally similar to the process for the log SDF (1) in that it includes a quadratic term. This term simplifies the process for the reciprocal of inflation by making the log of the conditional mean of $1 / \Pi_{t+1}$ the negative of the sum of the two state variables $\lambda_{t}$ and $\xi_{t}$. This in turn simplifies the pricing of short-term nominal bonds.

The real cash flow on a single-period nominal bond is simply $1 / \Pi_{t+1}$. Thus the price of the bond is given by $P_{1, t}^{\$}=\mathrm{E}_{t}\left[\exp \left\{m_{t+1}-\pi_{t+1}\right\}\right]$, so the log short-term nominal rate $y_{1, t+1}^{\$}=-\log \left(P_{1, t}^{\$}\right)$ is

$$
\begin{aligned}
y_{1, t+1}^{\$} & =-\mathrm{E}_{t}\left[m_{t+1}-\pi_{t+1}\right]-\frac{1}{2} \operatorname{Var}_{t}\left(m_{t+1}-\pi_{t+1}\right) \\
& =x_{t}+\lambda_{t}+\xi_{t}-\sigma_{m \pi} \psi_{t} .
\end{aligned}
$$

The log nominal short rate is the sum of the log real interest rate, the two state variables that drive expected log inflation, and a term that accounts for the correlation between shocks to inflation and shocks to the stochastic discount factor. This term, $-\sigma_{m \pi} \psi_{t}$, is the expected excess return on a single-period nominal bond over a singleperiod real bond so it measures the inflation risk premium at the short end of the term structure.

The log price of a $n$-period zero-coupon nominal bond is a linear-quadratic function of the vector of state variables:

$$
p_{n, t}^{\$}=A_{n}^{\$}+B_{x, n}^{\$} x_{t}+B_{\lambda, n}^{\$} \lambda_{t}+B_{\xi, n}^{\$} \xi_{t}+B_{\psi, n}^{\$} \psi_{t}+C_{\psi, n}^{\$} \psi_{t}^{2},
$$

where the coefficients $A_{n}^{\$}, B_{i, n}^{\$}$, and $C_{i, n}^{\$}$ solve a set of recursive equations given in the Appendix. From equation (10), $B_{x, 1}^{\$}=B_{\xi, 1}^{\$}=B_{\lambda, 1}^{\$}=-1, C_{z \psi, 1}^{\$}=\sigma_{m \pi}$, and the remaining coefficients are zero at $n=1$. 
Like risk premia in the real term structure, risk premia in the nominal term structure are linear in $\psi_{t}$. Intuitively, at times when inflation is procyclical - as might be the case if the macroeconomy moves along a stable Phillips Curve - nominal bond returns are countercyclical, making nominal bonds desirable hedges against business cycle risk. At times when inflation is countercyclical - as might be the case if the economy is affected by supply shocks or changing inflation expectations that shift the Phillips Curve in or out-nominal bond returns are procyclical and investors demand a positive risk premium to hold them.

\subsection{Pricing equities}

We want our model to fit the changing covariance of bonds and stocks, and so we must specify a process for the equity return within the model. One modelling strategy would be to specify a dividend process and solve for the stock return endogenously in the manner of Mamaysky (2002), Bekaert et al. (2005), and d'Addona and Kind (2006). However we adopt a simpler approach. Following Campbell and Viceira (2001), we model shocks to realized stock returns as a linear combination of shocks to the real interest rate and shocks to the log stochastic discount factor:

$$
r_{e, t+1}-\mathrm{E}_{t} r_{e, t+1}=\beta_{e x} \varepsilon_{x, t+1}+\beta_{e X} \varepsilon_{X, t+1}+\beta_{e m} \varepsilon_{m, t+1}+\varepsilon_{e, t+1},
$$

where $\varepsilon_{e, t+1}$ is an identically and independently distributed shock uncorrelated with all other shocks in the model. This shock captures movements in equity returns that are both unrelated to real interest rates and carry no risk premium because they are uncorrelated with the SDF.

Substituting (12) into the no-arbitrage condition $E_{t}\left[M_{t+1} R_{t+1}\right]=1$, the Appendix shows that the equity risk premium is given by

$$
\mathrm{E}_{t}\left[r_{e, t+1}-r_{1, t+1}\right]+\frac{1}{2} \operatorname{Var}_{t}\left(r_{e, t+1}-r_{1, t+1}\right)=\beta_{e x} \sigma_{x m}+\beta_{e X} \sigma_{X m}+\beta_{e m} \sigma_{m}^{2} .
$$

The equity premium depends not only on the direct sensitivity of stock returns to the SDF, but also on the sensitivity of stock returns to the real interest rate and the covariance of the real interest rate with the SDF.

Equation (12) does not attempt to capture heteroskedasticity in stock returns. Although such heteroskedasticity is of first-order importance for understanding stock 
prices, we abstract from it here in order to maintain the parsimony of our term structure model. Moreover, as Figure 1 shows, the stock-bond covariance and the stockbond beta move closely together, indicating that our assumption of homoskedastic stock returns is not overly restrictive for the purposes of studying the quantity of risk in nominal bonds.

The conditional covariance between the SDF and inflation also determines the covariance between the excess returns on real and nominal assets. Consider for example the conditional covariance between the real return on a one-period nominal bond and the real return on equities, both in excess of the return on a one-period real bond. This covariance is given by

$$
\operatorname{Cov}_{t}\left(r_{e, t+1}-r_{1, t+1}, y_{1, t+1}^{\$}-\pi_{t+1}-r_{1, t+1}\right)=-\left(\beta_{e x} \sigma_{x \pi}+\beta_{e m} \sigma_{m \pi}\right) \psi_{t},
$$

which moves over time and can change sign. This implies that we can identify the dynamics of the state variable $\psi_{t}$ from the dynamics of the conditional covariance between equities and nominal bonds as well as real bonds.

\section{Model Estimation}

\subsection{Data and estimation methodology}

The term structure model presented in Section 3 generates bond yields which are linear-quadratic functions of a vector of latent state variables. We now use this model to study the postwar history of yields on US Treasury nominal and inflation-indexed bonds. Since our state variables are not observable, and the observable series have a nonlinear dependence on the latent state variables, we obtain maximum likelihood estimates of our model's parameters via a nonlinear Kalman filter. Specifically, we use the unscented Kalman filter estimation procedure of Julier and Uhlmann (1997).

The unscented Kalman filter is a nonlinear Kalman filter which works through deterministic sampling of points in the distribution of the innovations to the state variables, does not require the explicit computation of Jacobians and Hessians, and captures the conditional mean and variance-covariance matrix of the state variables accurately up to a second-order approximation for any type of nonlinearity, and up to a third-order approximation when innovations to the state variables are Gaussian. 
Wan and van der Merwe (2001) describe in detail the properties of the filter and its practical implementation, and Binsbergen and Koijen (2008) apply the method to a prediction problem in finance. ${ }^{8}$

To implement the unscented Kalman filter, we specify a system of twelve measurement equations that relate observable variables to the vector of state variables. We sample the data at a quarterly frequency in order to minimize the impact of highfrequency noise in the measurement of some of our key variables - such as realized inflation - while keeping the frequency of observation reasonably high (Campbell and Viceira 2001, 2002). By not having to fit all the high-frequency monthly variation in the data, our estimation procedure can concentrate on uncovering the low-frequency movements in interest rates which our model is designed to capture.

Our first four measurement equations relate observable nominal bond yields to the vector of state variables, as in equation (11). We use yields on constant maturity 3month, 1-year, 3-year, and 10-year zero-coupon nominal bonds sampled at a quarterly frequency for the period 1953Q1-2009Q3. These data are spliced together from two sources. From 1953Q1-1961Q1 we sample quarterly from the monthly dataset developed by McCulloch and Kwon (1993), and from 1961Q2-2009Q3 we sample quarterly from the daily dataset constructed by Gürkaynak, Sack, and Wright (GSW 2006, updated through 2009). We assume that bond yields are measured with errors, which are uncorrelated with each other and with the structural shocks of the model.

Our fifth measurement equation, (7), relates the observed inflation rate to expected inflation and inflation volatility, plus measurement error. We use the CPI as our observed price index in this measurement equation. We complement this measurement equation with another one that uses data on the median forecast of GDP deflator inflation from the Survey of Professional Forecasters for the period 1968Q42009Q3. We relate this observed measure of expected inflation to the sum of equations (8) and (9) in our model plus measurement error.

The seventh measurement equation relates the observed yield on constant maturity Treasury inflation protected securities (TIPS) to the vector of state variables, via the

\footnotetext{
${ }^{8}$ Binsbergen and Koijen's application has linear measurement equations and nonlinear transition equations, whereas ours has linear transition equations and nonlinear measurement equations. The unscented Kalman filter can handle either case. We have also checked the robustness of our estimates by re-estimating our model using the "square root" variant of the filter, which has been shown to be more stable when some of the state variables follow heteroskedastic processes. This variant produces estimates which are extremely similar to the ones we report in the paper.
} 
pricing equation for real bonds (5). We obtain data on constant maturity zero-coupon 10-year TIPS dating back to the first quarter of 1999 from GSW (2008). Before 1999, we treat the TIPS yield as missing, which can easily be handled by the Kalman filter estimation procedure. As with nominal bond yields, we assume that real bond yields are measured with errors.

Figure 3 illustrates our real bond yield series. The decline in the TIPS yield since the year 2000, and the spike in the fall of 2008, are clearly visible in this figure. Campbell, Shiller, and Viceira (2009) document that this decline in the long-term real interest rate, and the subsequent sudden increase during the financial crisis, occurred in inflation-indexed bond markets around the world. In earlier data from the UK, long-term real interest rates were much higher on average during the 1980's and 1990's. Our model will explain such large and persistent variation in the TIPS yield primarily using persistent movements in the short-term real interest rate.

Our eighth measurement equation uses equity returns from the CRSP valueweighted index comprising the stocks traded in the NYSE, AMEX, and NASDAQ. This equation describes realized log equity returns $r_{e, t+1}$ using equations (12) and (13).

The last four measurement equations use the implications of our model for: (i) the conditional covariance between equity returns and real bond returns, (ii) the conditional covariance between equity returns and nominal bond returns, (iii) the conditional volatility of real bond returns, and (iv) the conditional volatility of nominal bond returns. The Appendix derives expressions for these time-varying conditional second moments, which are functions of $\psi_{t}$ and therefore help us filter this state variable. Following Viceira (2012), we construct the analogous realized second moments using high-frequency data. We obtain daily stock returns from CRSP and calculate daily nominal bond returns from daily GSW nominal yields from 1961Q2 onwards, and daily real bond returns from daily GSW real yields from 1999Q1 onwards. ${ }^{9}$ We then compute the variances and covariances realized over quarter $t$.

Realized variances and covariances in quarter $t$ are expected variances and covariances at quarter $t-1$, plus shocks realized in quarter $t$. Unfortunately we cannot treat such shocks as pure measurement error because they may be contemporaneously

\footnotetext{
${ }^{9}$ We calculate daily returns on the $n$ year bond from daily yields as $r_{n, t+1}=n y_{n, t}-$ $(n-1 / 264) y_{n, t+1}$. We assume there are 264 trading days in the year, or 22 trading days per month. Prior to 1961Q2, we calculate monthly returns from monthly McKullock-Kwon nominal yields, and calculate variances and covariances using a rolling 12-month return window.
} 
correlated with innovations to the state variables of our model. ${ }^{10}$ Accordingly we project realized variances and covariances onto information known at quarter $t-1$, and treat the fitted values as the conditional (expected) moments at quarter $t-1$ plus measurement error. For each realized variance and covariance, we use three pieces of information known at quarter $t-1$ : the lagged value of the realized variance or covariance, the 3-month nominal Treasury yield, and the spread between the 10-year nominal yield and the 3 -month nominal yield. Viceira (2012) shows these variables have strong predictive power for the realized second moments at quarter $t$. We also note that, because the realized second moments are persistent, the fitted values are quite similar to the realized second moments at quarter $t$.

The data used in these measurement equations are plotted in Figure 4 for real bonds and in Figure 5 for nominal bonds. The left panel of each figure shows the projected covariance between daily stock and bond returns, while the right panel shows the projected variance of daily bond returns. The thick lines in each panel show a smoothed version of the raw data.

Figure 5 shows that both the stock-nominal bond covariance series and the nominal bond variance series increase in the early 1970's and, most dramatically, in the early 1980 's. In the 1950's, and again in the 2000's, the stock-nominal bond covariance was negative, with downward spikes in the two recessions of the early 2000's and the late 2000's. Figure 4 shows that the stock-real bond covariance series and the real bond variance series follow patterns similar to those of nominal bonds for the overlapping sample period.

Our model has a large number of shocks, and we have found that freely estimating many of the covariances between these shocks does not materially affect the empirical results. Therefore, for parsimony we constrain some of these covariances to be zero. The unconstrained parameters are the covariances of all shocks with the stochastic discount factor $\left(\sigma_{x m}, \sigma_{X m}, \sigma_{\lambda m}, \sigma_{\Lambda m}, \sigma_{\xi m}, \sigma_{\psi m}, \sigma_{m \pi}\right)$; the covariances of the transitory component of expected inflation with realized inflation $\left(\sigma_{\xi \pi}\right)$ and the heteroskedastic shock to the real interest rate $\left(\sigma_{x \xi}\right)$; and the covariance of realized inflation with the heteroskedastic shock to the real interest rate $\left(\sigma_{x \pi}\right)$. In addition, recall that an observationally equivalent model can be obtained by multiplying $\psi_{t}$ and all covariances by -1 . Thus, without loss of generality we constrain the mean of $\psi_{t}$ to be positive.

With these constraints on the variance-covariance matrix, we allow freely esti-

${ }^{10}$ We thank an anonymous referee for pointing out this issue. 
mated risk premia on all the state variables. We allow correlations among real interest rates, realized inflation, and the transitory component of expected inflation, while imposing that the permanent component of expected inflation is uncorrelated with movements in the transitory state variables. This constraint is natural if longrun expected inflation is determined by central bank credibility, which depends on political economy considerations rather than business-cycle fluctuations in the economy. A likelihood ratio test of the constrained model cannot reject it against the fully parameterized model with all parameters estimated freely. Results from the fully parameterized model can be found in the Appendix.

\subsection{Parameter estimates}

Table 1 presents quarterly parameter estimates over the period 1953-2009 and their asymptotic standard errors, calculated numerically using the outer product method. The real interest rate is the most persistent state variable, with an autoregressive coefficient of 0.94 corresponding to a half life of 11 quarters. This persistence reflects the observed variability and persistence of TIPS yields. The nominal-real covariance and the transitory component of expected inflation are less persistent processes in our model, with half-lives of about 4 and 5 quarters respectively. Of course the model also includes a permanent component of expected inflation. If we model expected inflation as a single stationary $\mathrm{AR}(1)$ process, as we did in the first version of this paper, we find expected inflation to be more persistent than the real interest rate. All persistence coefficients are precisely estimated, with very small standard errors.

Table 1 shows large differences in the volatility of shocks to the state variables. The one-quarter conditional volatility of the homoskedastic shock to the annualized real interest rate is estimated to be about 53 basis points, and the average one-quarter conditional volatility of the heteroskedastic shock to the annualized real interest rate is estimated to be 82 basis points. The average one-quarter conditional volatility of the transitory component of annualized expected inflation is about 67 basis points, and the average one-quarter conditional volatility of annualized realized inflation is about 279 basis points. ${ }^{11}$ By contrast, the average one-quarter conditional volatilities of the shocks to the permanent component of expected inflation are very small. Of

\footnotetext{
${ }^{11}$ We compute the average conditional volatilities of the heteroskedastic shock to the real interest rate, the components of expected inflation, and realized inflation as $\left(\mu_{\psi}^{2}+\sigma_{\psi}^{2}\right)^{1 / 2}$ times the volatility of the underlying shocks. For example, we compute the average conditional volatility of realized
} 
course, the unconditional standard deviations of the real interest rate and the two components of expected inflation are much larger because of the high persistence of the processes; in fact, the unconditional standard deviation of the permanent component of expected inflation is undefined because this process has a unit root. With the exception of the volatility of the heteroskedastic shock to the permanent component of expected inflation, the volatility parameters for the real interest process and inflation are all precisely estimated with very small asymptotic standard errors.

Table 1 also reports the unrestricted correlations among the shocks and their asymptotic standard errors. We report correlations instead of covariances to facilitate interpretation. We compute their standard errors from those of the primitive parameters of the model using the delta method. The Appendix reports covariances and their asymptotic standard errors.

There is a correlation of over -0.16 between $\xi_{t}$ and $-m_{t}$ shocks. Although the correlation coefficient is not significant at the $5 \%$ level, the Appendix shows that the covariance is more precisely estimated. This negative correlation implies that the transitory component of expected inflation is countercyclical, generating a positive risk premium in the nominal term structure, when the state variable $\psi_{t}$ is positive; but transitory expected inflation is procyclical, generating a negative risk premium, when $\psi_{t}$ is negative. The absolute magnitude of the correlation between $\lambda_{t}$ and $-m_{t}$ shocks is larger at around -0.73 , implying that the risk premium for permanent shocks to expected inflation is larger than the risk premium for transitory shocks to expected inflation. However, this correlation has a very large standard error. Similarly, the magnitude of the correlation between $\Lambda_{t}$ and $-m_{t}$ shocks is relatively large, but the correlation is estimated quite imprecisely.

We also estimate a statistically insignificant and economically very small positive correlation between $\pi_{t}$ and $-m_{t}$ shocks. The point estimate implies that shortterm inflation risk is very small, and that nominal Treasury bills have a very small or zero inflation risk premium. In addition, we estimate a marginally statistically significant negative correlation of almost -0.25 between $x_{t}$ and $-m_{t}$ shocks, implying a time-varying term premium on real bonds that is positive when $\psi_{t}$ is positive.

Finally, we estimate a negative correlation of nearly -0.35 between $\psi_{t}$ and $-m_{t}$ shocks. While not statistically significant, this point estimate indicates that bond risk

inflation as $\left(\mu_{\psi}^{2}+\sigma_{\psi}^{2}\right)^{1 / 2} \sigma_{\pi}$, where recall that we have normalized $\sigma_{\pi}=1$. 
is countercyclical, rising in bad times. Since bond risk premia rise with the quantity of risk, this is consistent with the findings of Ludvigson and $\mathrm{Ng}$ (2009), who find evidence that bond risk premia are countercyclically related to macroeconomic factors. The remaining covariances are estimated to be very close to zero and statistically insignificant.

In the equity market, we estimate statistically insignificant small loadings of stock returns on shocks to the real interest rate $\left(\beta_{e x}\right.$ and $\left.\beta_{e X}\right)$, and a much larger and statistically significant positive loading on shocks to the negative of the log SDF $\left(\beta_{\text {em }}\right)$. Naturally this estimate implies a positive equity risk premium. ${ }^{12}$

\subsection{Fitted state variables}

How does our model interpret the economic history of the last 55 years? That is, what time series does it estimate for the underlying state variables that drive bond and stock prices? Figure 6 shows our estimates of the real interest rate $x_{t}$. The model estimates a process for the real interest rate that is high on average, with a spike in the early 1980's, and becomes more volatile and declining in the second half of the sample. Higher-frequency movements in the real interest rate were often countercyclical in this period, as we see the real rate falling in the recessions of the early 1970's, early 1990's, early 2000's, and at the end of our sample period in 200709. The real interest rate also falls around the stock market crash of 1987 . However there are important exceptions to this pattern, notably the very high real interest rate in the early 1980's, during Paul Volcker's campaign against inflation. Since the late 1990's the real interest rate generally tracks the TIPS yield, as shown in Figure 3. Thus the model attributes the history of long-dated TIPS yields mostly to changes in the short-term real rate $x_{t}$, with a supporting role for the state variable $\psi_{t}$.

Figure 7 plots the components of expected inflation. The permanent component of expected inflation, in the left panel, exhibits a familiar hump shape over the postwar

\footnotetext{
${ }^{12}$ However, the equity premium in the model is substantially lower than in the data. Our estimates imply a maximum Sharpe ratio of $15 \%$, while the Sharpe ratio for equities in our data is $35 \%$. In the Appendix, we provide estimates of the model where we constrain the maximum Sharpe ratio to be $50 \%$. This results in a substantially lower value of the likelihood function. The estimation routine prefers to trade a counterfactually low maximum Sharpe ratio to improve the model's fit along other dimensions. In particular, raising the Sharpe ratio creates counterfactually high bond return volatilities.
} 
period. It was low, even negative, in the mid-1950's, increased during the 1960's and 1970's, and reached a maximum value of about $10 \%$ in the first half of the 1980's. Since then, it has experienced a secular decline and remained close to $2 \%$ throughout the 2000's.

The transitory component of expected inflation, in the right panel, was particularly high in the late 1970's and 1980, indicating that investors expected inflation to decline gradually from a temporarily high level. The transitory component has been predominantly negative since then till almost the end of our sample period, implying that our model attributes the generally high levels of yield spreads during the second half of our sample period at least partly to investor pessimism about increases in future inflation. By estimating a generally negative transitory component of expected inflation, the model is also able to explain simultaneously the low average nominal short-term interest rate and the high average real short-term interest rate in the latter part of our sample period.

Finally, Figure 8 shows the time series of $\psi_{t}$. As we have noted, this variable is identified primarily through the covariance of stock returns and bond returns and the volatility of bond returns - both nominal and real. The state variable $\psi_{t}$ exhibits low volatility and an average close to zero in the period leading up to the late 1970's, with briefly negative values in the late 1950's, and an upward spike in the early 1970's. It becomes much more volatile starting in the late 1970's through the end of our sample period. It rises to large positive values in the early 1980's and stays predominantly positive through the 1980's and 1990's. However, in the late 1990's it switches sign and turns predominantly negative, with particularly large downward spikes in the period immediately following the recession of 2001 and in the fall of 2008, at the height of the financial crisis of 2007-09. Thus $\psi_{t}$ not only can switch sign, it has done so during the past ten years. Overall, the in-sample average for $\psi_{t}$ is positive.

The state variables we have estimated can be used to calculate fitted values for observed variables such as the nominal term structure, real term structure, realized inflation, analysts' median inflation forecast, and the realized second moments of bond and equity returns. We do not plot the histories of these fitted values to save space. They track the actual observed yields on nominal bonds, inflation forecasts, and the realized stock-nominal bond covariance very closely, and closely the yields on TIPS, realized inflation, and the rest of the realized second moments included in the estimation. In general, our model is rich enough that it does not require measurement errors with high volatility to fit the observed data on stock and bond prices. 


\section{$5 \quad$ Term Structure Implications}

\subsection{Moments of bond yields and returns}

Although our model fits the observed history of real and nominal bond yields, an important question is whether it must do so by inferring an unusual history of shocks, or whether the observed properties of interest rates emerge naturally from the properties of the model at the estimated parameter values. In order to assess this, Table 2 reports some important moments of bond yields and returns.

The table compares the sample moments in our historical data with moments calculated by simulating our model 1,000 times along a path that is 250 quarters (or 62 and a half years) long, and averaging time-series moments across simulations. Sample moments are shown in the first column and model-implied moments in the second column. The third column reports the fraction of simulations for which the simulated time-series moment is larger than the corresponding sample moment in the data. These numbers can be used as informal tests of the ability of the model to fit each sample moment. Although our model is estimated using maximum likelihood, these diagnostic statistics capture the spirit of the method of simulated moments (Duffie and Singleton 1993, Gallant and Tauchen 1996), which minimizes a quadratic form in the distance between simulated model-implied moments and sample moments. ${ }^{13}$

The first two rows of Table 2 report the sample and simulated means for nominal bond yield spreads, calculated using 3 and 10 year maturities, and the third and fourth rows look at the volatilities of these spreads. Our model provides a fairly good fit to average yield spreads, although it does understate both the average 3year spread (slightly) and the average 10-year spread (to a greater extent, therefore overstating the average concavity of the yield curve). A more serious problem for the model is that it systematically overstates the volatility of yield spreads, a problem that appears in almost all our 1,000 simulations.

The next four rows show how our model fits the means and standard deviations

\footnotetext{
${ }^{13}$ In Table 2 the short-term interest rate is a three-month rate and moments are computed using a three-month holding period. In the Appendix we report a table using a one-year short rate and holding period. This alternative table follows Cochrane and Piazzesi (2005), and shows us how our model fits lower frequency movements at the longer end of the yield curve. Results are comparable to those reported in Table 2.
} 
of realized excess returns on 3-year and 10-year nominal bonds. In order to calculate three-month realized returns from constant-maturity bond yields, we interpolate yields between the constant maturities we observe, doing this in the same manner for our historical data and for simulated data from our models. Just as with yield spreads, the model provides a good fit to mean excess returns. It overstates the volatility of excess returns on 3-year bonds but provides a good fit for the volatility of 10-year bonds.

The next four rows of the table summarize our model description of TIPS yields. The model generates an average TIPS yield that is somewhat higher than the observed average. We do not believe this is a serious problem, as our estimates imply higher real interest rates earlier in our sample period, before TIPS were issued, than in the period since 1997 over which we measure the average TIPS yield. Thus the discrepancy may result in part from the short and unrepresentative period over which we measure the average TIPS yield in the data.

The model implies a small negative average real yield spread and a small positive average realized excess return. The difference between these two statistics reflects the effect of Jensen's Inequality; equivalently, it is the result of convexity in long-term bonds. The positive average risk premium results from our negative estimate of $\rho_{x m}$ in Table 1, which implies that the real interest rate is countercyclical on average.

\subsection{Risk premia and the yield curve}

In our model, all time variation in bond risk premia is driven by variation in bond risk, not by variation in the aggregate price of risk. It follows that long bond risk premia are linear in the state variable $\psi_{t}$. Figure 9 illustrates this fact. The left panel plots the simulated expected excess return on 3-year and 10-year nominal bonds over 3-month Treasury bills against $\psi_{t}$. The right panel of the figure shows the term structure of risk premia as $\psi_{t}$ varies from its sample mean to its sample minimum and maximum. Risk premia spread out rapidly as maturity increases, and 10-year risk premia vary from -45 to 115 basis points.

The full history of our model's 10-year term premium is illustrated in Figure 10. The figure shows fairly stable risk premia of about $0.2 \%$ during the 1950's and 1960's, a spike in the early 1970's, and a run up later in the 1970's to a peak of about $1.15 \%$ in the early 1980's. A long decline in risk premia later in the sample period was 
accentuated around the recession of the early 2000's and during the financial crisis of 2007-09, bringing the risk premium to its sample minimum of $-0.45 \%$. This time series reflects the shape in the nominal-real covariance $\psi_{t}$ illustrated in Figure 8.

An important question is how the shape of the yield curve responds to these variations in risk premia. To isolate the effect of changing $\psi_{t}$, Figure 11 plots the log real and nominal yield curves generated by our model when $\psi_{t}$ is at its in-sample mean, maximum, and minimum, while all other state variables are at their in-sample means. Thus the central line describes the yield curve - real or nominal - generated by our model when all state variables are evaluated at their in-sample mean. For simplicity we will refer to this curve as the "mean log yield curve." 14

In both panels of Figure 11, increasing $\psi_{t}$ from the sample mean to the sample maximum raises intermediate-term yields and lowers long-term yields, while decreasing $\psi_{t}$ to the sample minimum lowers both intermediate-term and long-term yields. Thus $\psi_{t}$ alters the concavity of both the real and nominal yield curves.

The impact of $\psi_{t}$ on the concavity of the nominal yield curve results from two features of our model. First, nominal bond risk premia increase with maturity rapidly at intermediate maturities and slowly at longer maturities because intermediate maturities are exposed both to transitory and permanent shocks to expected inflation. When $\psi_{t}$ is positive, this generates a steep yield curve at shorter maturities, and a flatter one at longer maturities. When $\psi_{t}$ changes sign, however, the difference in risk prices pulls intermediate-term yields down more strongly than long-term yields.

Second, when $\psi_{t}$ is far from zero bond returns are unusually volatile, and through Jensen's Inequality this lowers the bond yield that is needed to deliver any given expected simple return. This effect is much stronger for long-term bonds; in the terminology of the fixed-income literature, these bonds have much greater "convexity" than short- or intermediate-term bonds. Therefore extreme values of $\psi_{t}$ tend to lower long-term bond yields relative to intermediate-term yields.

Similar effects operate in the real term structure. Real bond risk premia are highly sensitive to $\psi_{t}$ at intermediate maturities because real interest rate variation

\footnotetext{
${ }^{14}$ Strictly speaking this is a misnomer for two reasons. First, the log real and nominal yield curves are non-linear functions of the vector of state variables. Second, the unconditional mean of the $\log$ nominal yield curve is not even defined, since one of the state variables follows a random walk. Thus at most we can compute a mean nominal yield curve conditional on initial values for the state variables.
} 
is transitory, and long-term real bonds have high convexity so their yields are driven down by high levels of bond volatility.

In the Appendix, we conduct similar analyses of term structure responses to our model's other state variables. Real interest rate shocks have highly persistent effects on both the real and nominal yield curve, while the permanent component of expected inflation shifts the nominal yield curve up and down, and the transitory component of expected inflation changes the slope of the nominal yield curve. These results can be related to Litterman and Scheinkman's (1991) "level", "slope", and "curvature" factors. In our model, the covariance of nominal and real variables $\psi_{t}$ primarily drives the curvature factor while the other state variables primarily move the level and slope factors. Thus our model suggests that the curvature factor is likely to be the best proxy for bond risk premia.

An empirical result of this sort has been reported by Cochrane and Piazzesi (CP, 2005). Using econometric methods originally developed by Hansen and Hodrick (1983), and implemented in the term structure context by Stambaugh (1988), CP show that a single linear combination of forward rates is a good predictor of excess bond returns at a wide range of maturities. CP work with a 1-year holding period and a 1-year short rate. They find that bond risk premia are high when intermediateterm interest rates are high relative to both shorter-term and longer-term rates; that is, they are high when the yield curve is strongly concave.

Our model interprets this phenomenon as the result of changes in the nominal-real covariance $\psi_{t}$. As $\psi_{t}$ increases, the risk premiums for both components of expected inflation rise. This strongly increases the intermediate-term yield, but it has a damped or even perverse effect on long-term yields because these yields respond only to the permanent component of expected inflation and the convexity of long bonds causes their yields to fall with volatility. Thus the best predictor of excess bond returns is the intermediate-term yield relative to the average of short- and long-term yields.

\subsection{The predictability of bond returns}

Despite this promising qualitative pattern, the predictability of bond returns is small in our model. The bottom panel of Table 2 illustrates this point. In the first three rows we report the standard deviations of true expected 3-month excess returns within our model. Our model implies an annualized standard deviation for the expected 
excess return on 3-year bonds of about 12 basis points, and for the expected excess return on 10-year bonds of about 19 basis points. ${ }^{15}$ This variation is an order of magnitude smaller than the annualized standard deviations of realized excess bond returns, implying that the true explanatory power of predictive regressions in our model is tiny. There is also modest variability of about 14 basis points in the true expected excess returns on TIPS.

The next three rows report the standard deviations of fitted values of CampbellShiller (1991, CS) predictability regressions of annualized nominal bond excess returns onto yield spreads of the same maturity at the beginning of the holding period. The standard deviations in the data are 104 basis points for 3-year bonds, and 251 basis points for 10-year bonds. These numbers are considerably larger than the true variability of expected excess returns in our model, implying that our model cannot match the behavior of these predictive regressions.

In artificial data generated by our model, predictive regressions deliver fitted values that are considerably more volatile than the true expected excess returns. The reason for this counterintuitive behavior is that there is important finite-sample bias in the CS regression coefficients of the sort described by Stambaugh (1999). In the case of regressions of excess bond returns on yield spreads, by contrast with the better known case of regressions of excess stock returns on dividend yields, the Stambaugh bias is negative (Bekaert, Hodrick, and Marshall 1997). In our full model, where the true regression coefficient is positive but close to zero, the Stambaugh bias increases the standard deviation of fitted values by generating spurious negative coefficients. Nonetheless, the standard deviation of fitted values in the model is still considerably smaller than in the data.

We obtain more promising results using a procedure that approximates the approach of Cochrane and Piazzesi (2005, CP). We regress excess bond returns on 1-, 3 -, and 5-year forward rates at the beginning of the holding period, and report the standard deviations of fitted values. ${ }^{16}$ This procedure generates comparable standard deviations of fitted values in the model and in the data, at least for predicting excess 3 -year bond returns. Once again, however, this finding is largely driven by small-

\footnotetext{
${ }^{15}$ Yield interpolation for 3-month returns may exaggerate the evidence for predictability; however the same yield interpolation is used for simulated data from our models. We have used our simulations to examine the effect of interpolation. We find that interpolation does slightly increase measured bond return predictability, but the effect is modest.

${ }^{16}$ Cochrane and Piazzesi impose proportionality restrictions across the regressions at different maturities, but we do not do this here.
} 
sample bias as the fitted values in the model have a much higher standard deviation than the true expected excess returns.

These results show that although our model does generate time-varying bond risk premia, the implied variation in risk premia is smaller and has a different time-series pattern from that implied by CS and CP regressions. In the CS case, the difference in time-series behavior can be understood visually by comparing the history of the yield spread with the history of the model-implied bond risk premium shown in Figure 10. The former has a great deal of business-cycle variation, while the latter has a hump shape with a long secular decline from the early 1980's through the late 2000's. The fitted value from a CP regression lines up somewhat better with the model-implied bond risk premium, but it too spikes up in the recessions of the early 1990's and early 2000's in a way that has no counterpart in Figure 10.

We have explored an extension of our model that allows for time-variation in the aggregate price of risk, identifying this time-variation explicitly with the yield spread as in Wachter (2006) and others. This extension allows the model to explain much more of the observed variation in bond risk premia, perhaps unsurprisingly given prior results in the literature. However, the low-frequency variation in the bond risk premium generated by changing bond risk remains present in that more complicated framework.

\section{Conclusion}

We have argued that term structure models must confront the fact that the covariances between nominal and real bond returns, on the one hand, and stock returns, on the other, have varied substantially over time and have changed sign. Analyses of asset allocation traditionally assume that broad asset classes have a stable structure of risk over time; our empirical results imply that for bonds at least, this assumption is seriously misleading.

We have added a changing covariance, which can change sign, to an otherwise standard term structure model with identifiable macroeconomic state variables. In our model real and nominal bond returns are driven by four factors: the real interest rate, transitory and permanent components of expected inflation, and a state variable that governs the covariances of inflation and the real interest rate with the stochastic 
discount factor. The model implies that the risk premia of nominal bonds should have changed over the decades because of changes in the covariance between inflation and the real economy. The model predicts positive nominal bond risk premia in the early 1980's, when bonds covaried strongly with stocks, and negative risk premia in the 2000's and particularly during the downturn of 2007-09, when bonds hedged equity risk.

Our model is consistent with the qualitative finding of Cochrane and Piazzesi (2005) that a tent-shaped linear combination of forward rates, with a peak at about 3 years, predicts excess bond returns at all maturities better than maturity-specific yield spreads. Since the model has a constant price of bond risk and explains risk premia only from time-variation in the quantity of bond risk, it does not replicate the high explanatory power of regressions that predict excess US Treasury bond returns from yield spreads and forward rates. However, the results do suggest that timevarying bond risk is important in understanding movements in bond risk premia, particularly at low frequencies.

We interpret our results as posing an important new challenge to the asset pricing literature. A successful asset pricing model should jointly explain the time-variation in bond and stock risk premia along with the time-variation in the comovements of bond and stock returns. Our model is a first attempt to do this, but it does not reconcile the changing second moments of bond and stock returns with high-frequency variation in bond risk premia captured by the shape of the yield curve. We hope that future term structure research will address the challenge by extending the model presented here.

There are a number of ways in which this can be done. First and most obviously, one can allow for changes in risk aversion, or the volatility of the stochastic discount factor, following Duffee (2002), Dai and Singleton (2002), Bekaert, Engstrom, and Grenadier (2005), Wachter (2006), Buraschi and Jiltsov (2007), and Bekaert, Engstrom, and Xing (2009).

Second, one can model changing second moments in stock returns, possibly deriving those returns from primitive assumptions on the dividend process, as in the recent literature on affine models of stock and bond pricing (Mamaysky 2002, Bekaert, Engstrom, and Grenadier 2005, d'Addona and Kind 2006, Bekaert, Engstrom, and Xing 2009).

Third, one can allow both persistent and transitory variation in the nominal-real 
covariance, as we have done for expected inflation. This might allow our model to better fit both the secular trends and cyclical variation in the realized covariance between bonds and stocks.

Fourth, one can consider other theoretically motivated proxies for the stochastic discount factor. An obvious possibility is to look at realized or expected future consumption growth, as in recent papers on consumption-based bond pricing by Piazzesi and Schneider (2006), Eraker (2008), Hasseltoft (2009), Lettau and Wachter (2011), and Bansal and Shaliastovich (2013). A disadvantage of this approach is that consumption is not measured at high frequency, so one cannot use high-frequency data to track a changing covariance between bond returns and consumption growth.

It will also be interesting to estimate our model using data from other countries, for example the UK, where inflation-indexed bonds have been actively traded since the mid-1980's. Evidence of bond return predictability is considerably weaker outside the US (Bekaert, Hodrick, and Marshall 2001, Campbell 2003) and may better fit the predictability generated by our model.

Finally, it is important to better understand the monetary and macroeconomic determinants of the bond-stock covariance. Within a new Keynesian paradigm, one possibility is that a positive covariance corresponds to an environment in which the Phillips Curve is unstable, perhaps because supply shocks are hitting the economy or the central bank lacks anti-inflationary credibility, while a negative covariance reflects a stable Phillips Curve. It would be desirable to use data on inflation and output, and a structural macroeconomic model, to explore this interpretation.

The connection between the bond-stock covariance and the state of the macroeconomy should be of special interest to central banks. Many central banks use the breakeven inflation rate, the yield spread between nominal and inflation-indexed bonds, as an indicator of their credibility. The bond-stock covariance may be appealing as an additional source of macroeconomic information. 


\section{References}

Ahn, Dong-Hyun, Robert F. Dittmar, and A. Ronald Gallant, 2002, "Quadratic Term Structure Models: Theory and Evidence", Review of Financial Studies $15,243-288$.

Ang, Andrew, Geert Bekaert, and Min Wei, 2008, "The Term Structure of Real Rates and Expected Inflation", Journal of Finance 63, 797-849.

Backus, David K. and Jonathan H. Wright, 2007, "Cracking the Conundrum", Brookings Papers on Economic Activity 1, 293-316.

Baele, Lieven, Geert Bekaert, and Koen Inghelbrecht, 2010, "The Determinants of Stock and Bond Return Comovements", Review of Financial Studies 23, 23742428 .

Bansal, Ravi and Ivan Shaliastovich, 2013, "A Long-Run Risks Explanation of Predictability Puzzles in Bond and Currency Markets", Review of Financial Studies $26,1-33$.

Bansal, Ravi, and Amir Yaron, 2004, "Risks for the Long Run: A Potential Resolution of Asset Pricing Puzzles," Journal of Finance 59, 1481-1509.

Bansal, Ravi and Hao Zhou, 2002, "Term Structure of Interest Rates with Regime Shifts", Journal of Finance 57, 1997-2043.

Barsky, Robert B., 1989, "Why Don't the Prices of Stocks and Bonds Move Together?", American Economic Review 79, 1132-1145.

Beaglehole, David R. and Mark S. Tenney, 1991, "General Solutions of Some Interest Rate-Contingent Claim Pricing Equations", Journal of Fixed Income, September, 69-83.

Bekaert, Geert, Eric Engstrom, and Steve Grenadier, 2010, "Stock and Bond Returns with Moody Investors", Journal of Empirical Finance 17, 867-894.

Bekaert, Geert, Eric Engstrom, and Yuhang Xing, 2009, "Risk, Uncertainty, and Asset Prices", Journal of Financial Economics 91, 59-82.

Bekaert, Geert, Robert J. Hodrick, and David A. Marshall, 1997, "On Biases in Tests of the Expectations Hypothesis of the Term Structure of Interest Rates", Journal of Financial Economics 44, 309-348. 
Bekaert, Geert, Robert J. Hodrick, and David A. Marshall, 2001, "Peso Problem Explanations for Term Structure Anomalies", Journal of Monetary Economics $48,241-270$.

Binsbergen, Jules H. van and Ralph S.J. Koijen, 2008, "The Impact of Non-Linearities in Present-Value Models," unpublished paper, Stanford University and University of Chicago.

Buraschi, Andrea, Anna Cieslak, and Fabio Trojani, 2008, "Correlation Risk and the Term Structure of Interest Rates", unpublished paper, Imperial College London and University of St. Gallen.

Buraschi, Andrea and Alexei Jiltsov, 2007, "Habit Formation and Macroeconomic Models of the Term Structure of Interest Rates", Journal of Finance 62, 30093063 .

Campbell, John Y., 1987, "Stock Returns and the Term Structure", Journal of Financial Economics 18, 373-399.

Campbell, John Y., 2003, "Consumption-Based Asset Pricing", Chapter 13 in George Constantinides, Milton Harris, and Rene Stulz eds. Handbook of the Economics of Finance Vol. IB, North-Holland, Amsterdam, 803-887.

Campbell, John Y. and John Ammer, 1993, "What Moves the Stock and Bond Markets? A Variance Decomposition for Long-Term Asset Returns", Journal of Finance 48, 3-37.

Campbell, John Y., Y. Lewis Chan, and Luis M. Viceira, 2003, "A Multivariate Model of Strategic Asset Allocation", Journal of Financial Economics 67, 4180 .

Campbell, John Y. and Robert J. Shiller, 1991, "Yield Spreads and Interest Rate Movements: A Bird's Eye View", Review of Economic Studies 58, 495-514.

Campbell, John Y., Robert J. Shiller, and Kermit L. Schoenholtz, 1983, "Forward Rates and Future Policy: Interpreting the Term Structure of Interest Rates", Brookings Papers on Economic Activity 173-217, Spring 1983.

Campbell, John Y., Robert J. Shiller, and Luis M. Viceira, 2009, "Understanding Inflation-Indexed Bond Markets", Brookings Papers on Economic Activity 79120, Spring 2009. 
Campbell, John Y., Adi Sunderam, and Luis M. Viceira, 2013, "Appendix to Inflation Bets or Deflation Hedges? The Changing Risks of Nominal Bonds", available online at http://kuznets.fas.harvard.edu/ campbell/papers.html.

Campbell, John Y. and Luis M. Viceira, 2001, "Who Should Buy Long-Term Bonds?", American Economic Review 91, 99-127.

Campbell, John Y. and Luis M. Viceira, 2002, Strategic Asset Allocation: Portfolio Choice for Long-Term Investors, Oxford University Press, New York, NY.

Christiansen, Charlotte and Angelo Ranaldo, 2007, "Realized Bond-Stock Correlation: Macroeconomic Announcement Effects", Journal of Futures Markets 27, 439-469.

Cochrane, John H. and Monika Piazzesi, 2005, "Bond Risk Premia", American Economic Review 95, 138-160.

Cogley, Timothy, Giorgio E. Primiceri, and Thomas J. Sargent, 2010, "Inflation-Gap Persistence in the US", American Economic Journal: Macroeconomics 2, 43-69.

Constantinides, George M., 1992, "A Theory of the Nominal Term Structure of Interest Rates", Review of Financial Studies 5, 531-552.

Cox, John, Jonathan E. Ingersoll, Jr. and Stephen A. Ross, 1985, "A Theory of the Term Structure of Interest Rates", Econometrica 53, 385-408.

d'Addona, Stefano and Axel H. Kind, 2006, "International Stock-Bond Correlations in a Simple Affine Asset Pricing Model", Journal of Banking and Finance 30, $2747-2765$.

Dai, Qiang and Kenneth Singleton, 2002, "Expectations Puzzles, Time-Varying Risk Premia, and Affine Models of the Term Structure", Journal of Financial Economics 63, 415-442.

David, Alexander and Pietro Veronesi, 2010, "What Ties Return Volatilities to Price Valuations and Fundamentals?", unpublished paper, University of Calgary and University of Chicago.

Duffee, Greg, 2002, "Term Premia and Interest Rate Forecasts in Affine Models", Journal of Finance 57, 405-443. 
Duffie, Darrell and Rui Kan, 1996, "A Yield-Factor Model of Interest Rates", Mathematical Finance 6, 379-406.

Duffie, Darrell and Kenneth J. Singleton, 1993, "Simulated Moments Estimation of Markov Models of Asset Prices", Econometrica 61, 929-952.

Engle, Robert F., 1982, "Autoregressive Conditional Heteroskedasticity with Estimates of the Variance of UK Inflation", Econometrica 50, 987-1008.

Eraker, Bjørn, 2008, "Affine General Equilibrium Models," Management Science 54, 2068-2080.

Fama, Eugene F., 2006, "The Behavior of Interest Rates" Review of Financial Studies 19, 359-379.

Fama, Eugene F. and Robert R. Bliss, 1987, "The Information in Long-Maturity Forward Rates", American Economic Review 77, 680-692.

Fama, Eugene F. and Kenneth R. French, 1993, "Common Risk Factors in the Returns on Stocks and Bonds", Journal of Financial Economics 33, 3-56.

Gallant, Ronald and George Tauchen, 1996, "Which Moments to Match", Econometric Theory 12, 657-681.

Garcia, Rene and Pierre Perron, 1996, "An Analysis of the Real Interest Rate under Regime Shifts", Review of Economics and Statistics 78, 111-125.

Gray, Stephen F., 1996, "Modelling the Conditional Distribution of Interest Rates as a Regime-Switching Process", Journal of Financial Economics 42, 27-62.

Greenwood, Robin and Dimitri Vayanos, 2012, "Bond Supply and Excess Bond Returns", unpublished paper, Harvard University and London School of Economics.

Guidolin, Massimo and Allan Timmermann, 2006, "An Econometric Model of Nonlinear Dynamics in the Joint Distribution of Stock and Bond Returns", Journal of Applied Econometrics 21, 1-22.

Gurkaynak, Refet S., Brian Sack, and Jonathan H. Wright, 2006, "The U.S. Treasury Yield Curve: 1961 to the Present", FEDS working paper 2006-28, available online at http://www.federalreserve.gov/pubs/feds/2006/200628. 
Gurkaynak, Refet S., Brian Sack, and Jonathan H. Wright, 2008, "The TIPS Yield Curve and Inflation Compensation", FEDS working paper 2008-05, available online at http://www.federalreserve.gov/pubs/feds/2008/200805.

Hansen, Lars P. and Robert J. Hodrick, 1983, "Risk Averse Speculation in the Forward Foreign Exchange Market: An Econometric Analysis of Linear Models", in J. Frenkel (ed.) Exchange Rates and International Macroeconomics, University of Chicago Press, Chicago.

Hasseltoft, Henrik, 2009, "The 'Fed-Model' and the Changing Correlation of Stock and Bond Returns: An Equilibrium Approach," unpublished paper, Stockholm School of Economics.

Julier, S. and J. Uhlmann, 1997, "A New Extension of the Kalman Filter to Nonlinear Systems", in Proceedings SPIE Signal Processing, Sensor Fusion, and Target Recognition VI, 3068, 182-193.

Krishnamurthy, Arvind and Annette Vissing-Jorgensen, 2012, "The Aggregate Demand for Treasury Debt", Journal of Political Economy 120, 233-267.

Lettau, Martin and Jessica Wachter, 2011, "The Term Structures of Equity and Interest Rates", Journal of Financial Economics 101, 90-113.

Li, Lingfeng, 2002, "Macroeconomic Factors and the Correlation of Stock and Bond Returns", Yale ICF Working Paper 02-46.

Litterman, Robert and Jose Scheinkman, 1991, "Common Factors Affecting Bond Returns", Journal of Fixed Income, June.

Ludvigson, Sydney and Serena Ng, 2009, "Macro Factors in Bond Risk Premia", Review of Financial Studies 22, 5027-5067.

Mamaysky, Harry, 2002, "Market Prices of Risk and Return Predictability in a Joint Stock-Bond Pricing Model", Yale ICF Working Paper 02-25.

McCulloch, J. Huston and H. Kwon, 1993, "US Term Structure Data, 1947-1991", Working Paper 93-6, Ohio State University.

Mishkin, Frederic S., 1990, "The Information in the Longer Maturity Term Structure About Future Inflation", Quarterly Journal of Economics 105, 815-828. 
Piazzesi, Monika and Martin Schneider, 2006, "Equilibrium Yield Curves", in D. Acemoglu, K. Rogoff, and M. Woodford (eds.) NBER Macroeconomics Annual 2006, 317-363, MIT Press, Cambridge, MA.

Realdon, Marco, 2006, "Quadratic Term Structure Models in Discrete Time", Finance Research Letters 3, 277-289.

Rudebusch, Glenn D. and Tao Wu, 2007, "Accounting for a Shift in Term Structure Behavior with No-Arbitrage and Macro-Finance Models", Journal of Money, Credit, and Banking 39, 395-422.

Sangvinatsos, Antonios and Jessica A. Wachter, 2005, "Does the Failure of the Expectations Hypothesis Matter for Long-Term Investors?", Journal of Finance $60,179-230$.

Shiller, Robert J. and Andrea Beltratti, 1992,"Stock Prices and Bond Yields: Can Their Comovements Be Explained in Terms of Present Value Models?", Journal of Monetary Economics 30, 25-46.

Stambaugh, Robert F., 1988, "The Information in Forward Rates: Implications for Models of the Term Structure", Journal of Financial Economics 21, 41-70..

Stambaugh, Robert F., 1999, "Predictive Regressions", Journal of Financial Economics 54:375-421, 1999.

Stock, James H. and Mark W. Watson, 2007, "Why Has U.S. Inflation Become Harder to Forecast?", Journal of Money, Credit, and Banking 39 (Supplement), $3-33$.

Viceira, Luis M., 2012, "Bond Risk, Bond Return Volatility, and the Term Structure of Interest Rates", International Journal of Forecasting 28, 97-117.

Wachter, Jessica A., 2006, "A Consumption-Based Model of the Term Structure of Interest Rates", Journal of Financial Economics 79, 365-399.

Wan, Eric A. and Rudolph van der Merwe, 2001, "The Unscented Kalman Filter", Chapter 7 in Simon Haykin ed., Kalman Filtering and Neural Networks, Wiley, New York, NY. 
Table 1: Parameter estimates.

\begin{tabular}{|c|c|c|}
\hline \multicolumn{3}{|c|}{ Parameter Estimates } \\
\hline Parameter & Estimate & Std Err \\
\hline$\mu_{x} \times 10^{3}$ & 9.217 & 0.642 \\
\hline$\mu_{\psi} \times 10^{3}$ & 4.815 & 1.050 \\
\hline$\phi_{x}$ & 0.938 & 0.005 \\
\hline$\phi_{\xi}$ & 0.880 & 0.008 \\
\hline$\phi_{\psi}$ & 0.847 & 0.032 \\
\hline$\sigma_{m} \times 10^{2}$ & 8.015 & 4.150 \\
\hline$\sigma_{X} \times 10^{3}$ & 1.319 & 0.100 \\
\hline$\sigma_{x} \times 10^{1}$ & 2.919 & 0.407 \\
\hline$\sigma_{\lambda} \times 10^{4}$ & 1.443 & 183.660 \\
\hline$\sigma_{\Lambda} \times 10^{4}$ & 2.776 & 0.088 \\
\hline$\sigma_{\xi} \times 10^{1}$ & 2.415 & 0.352 \\
\hline$\sigma_{\psi} \times 10^{3}$ & 5.058 & 2.099 \\
\hline$\beta_{e X}$ & 1.133 & 3.980 \\
\hline$\beta_{e x} \times 10^{2}$ & 0.548 & 4.535 \\
\hline$\beta_{e m} \times 10^{2}$ & 9.538 & 5.169 \\
\hline$\rho_{x \xi}$ & 0.000 & 0.075 \\
\hline$\rho_{x m}$ & -0.246 & 0.136 \\
\hline$\rho_{X m} \times 10^{2}$ & 0.007 & 0.017 \\
\hline$\rho_{x \pi}$ & 0.000 & 0.620 \\
\hline$\rho_{\lambda m}$ & -0.732 & 0.933 \\
\hline$\rho_{\Lambda m}$ & -0.451 & 0.844 \\
\hline$\rho_{\xi m}$ & -0.163 & 0.158 \\
\hline$\rho_{\xi \pi}$ & -0.035 & 0.826 \\
\hline$\rho_{\psi m}$ & -0.347 & 0.238 \\
\hline$\rho_{m \pi}$ & 0.009 & 0.107 \\
\hline
\end{tabular}


Table 2: Sample and Implied Moments. Yield spreads (YS) are calculated over the 3mo yield. Realized excess returns (RXR) are calculated over a 3mo holding period, in excess of the 1yr yield. Units are annualized percentage points. Simulation columns report means across 1000 replications, each of which simulates a timeseries of 250 quarters. The $\sigma(\widehat{C P})$ row reports the standard deviation of the fitted values from a CochranePiazzesi style regression of RXR on the 1-, 3-, and 5-yr forward rates at the beginning of the holding period. The $\sigma(\widehat{C S})$ row reports the standard deviation of the fitted values from a Campbell-Shiller style regression of RXR on the same-maturity YS at the beginning of the holding period. In the rightmost column we report the fraction of simulation runs where the simulated value exceeds the data value. ${ }^{\dagger}$ Data moments for the $10 \mathrm{yr}$ return require $117 \mathrm{mo}$ yields. We interpolate the $117 \mathrm{mo}$ yield linearly between the $5 \mathrm{yr}$ and the $10 \mathrm{yr} \ddagger$ TIPS entries refer to the 10yr spliced TIPS yield. We have this data 1/1999-9/2009.

\begin{tabular}{cccc}
\hline \hline \multicolumn{4}{c}{ Sample and Implied Moments } \\
\hline \hline Moment & Actual Data & Model & Above \\
\hline 3yr YS mean & 0.62 & 0.46 & 0.36 \\
10yr YS mean & 1.15 & 0.67 & 0.31 \\
3yr YS stdev & 0.45 & 0.67 & 0.98 \\
10yr YS stdev & 0.70 & 1.36 & 1.00 \\
3yr RXR mean & 1.17 & 0.98 & 0.40 \\
10yr RXR mean & 2.21 & 1.46 & 0.30 \\
3yr RXR stdev & 4.37 & 6.35 & 1.00 \\
10yr RXR stdev & 11.16 & 11.07 & 0.44 \\
10yr TIPS yield mean & $2.58^{\ddagger}$ & 3.52 & 0.97 \\
10yr TIPS YS mean & & -0.16 & \\
10yr TIPS RXR mean & & 0.16 & \\
10yr TIPS RXR stdev & & 9.90 & \\
\hline
\end{tabular}

\begin{tabular}{cccc}
\hline \hline \multicolumn{4}{c}{ Predictive Regressions } \\
\hline Moment & Actual Data & Model & Above \\
\hline 3yr EXR stdev & & 0.12 & \\
10yr EXR stdev & & 0.19 & \\
10yr TIPS EXR stdev & & 0.14 & \\
\hline 3yr RXR $\sigma(\widehat{C S})$ & 1.04 & 0.40 & 0.04 \\
10yr RXR $\sigma(\widehat{C S})$ & $2.51^{\dagger}$ & 0.68 & 0.01 \\
10yr TIPS RXR $\sigma(\widehat{C S})$ & & 0.60 & \\
\hline 3yr RXR $\sigma(\widehat{C P})$ & 0.79 & 0.81 & 0.48 \\
10yr RXR $\sigma(\widehat{C P})$ & $2.06^{\dagger}$ & 1.41 & 0.17 \\
\hline
\end{tabular}




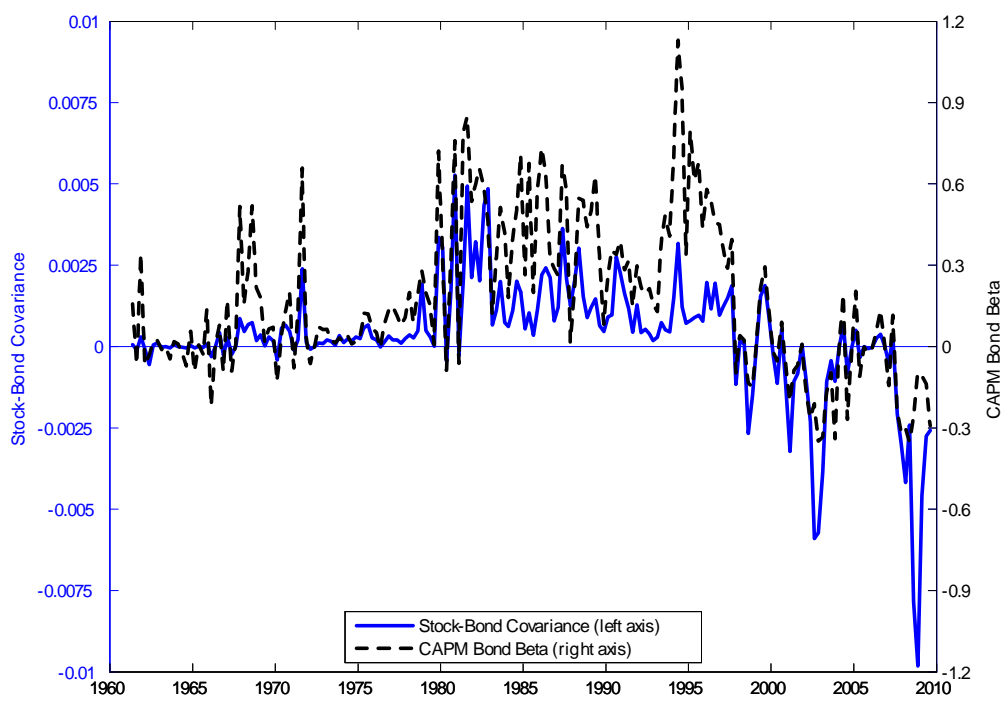

Figure 1: Time series of the stock-bond covariance and the CAPM $\beta$ of the 10-year nominal bond.

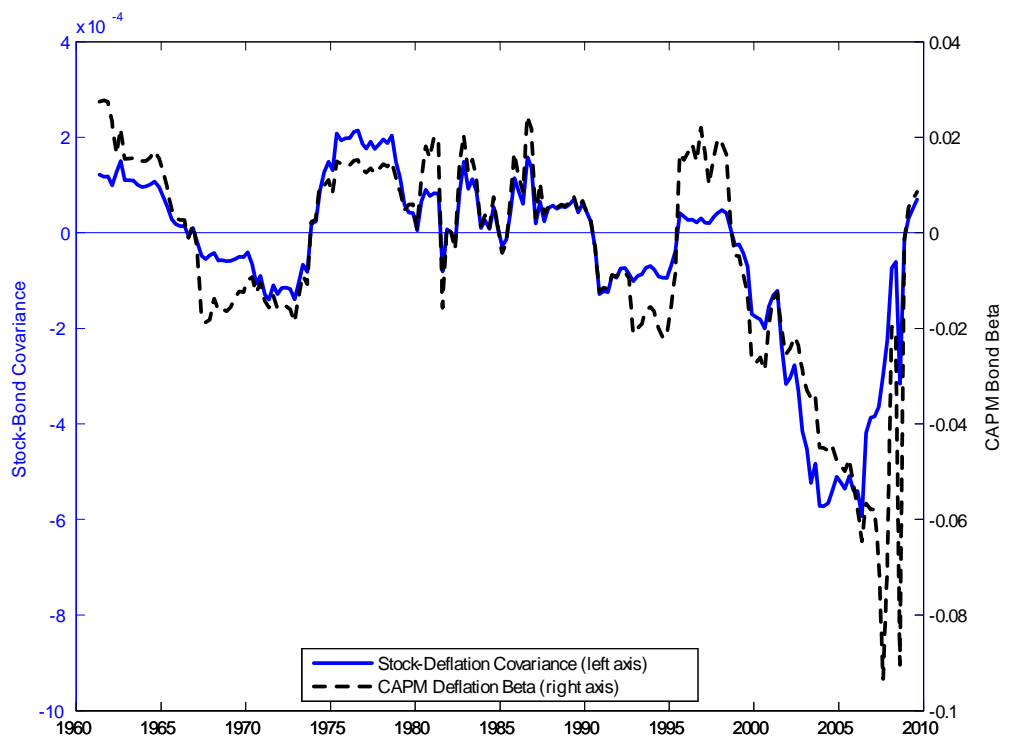

Figure 2: Time series of the stock-deflation covariance and the CAPM $\beta$ of deflation. 


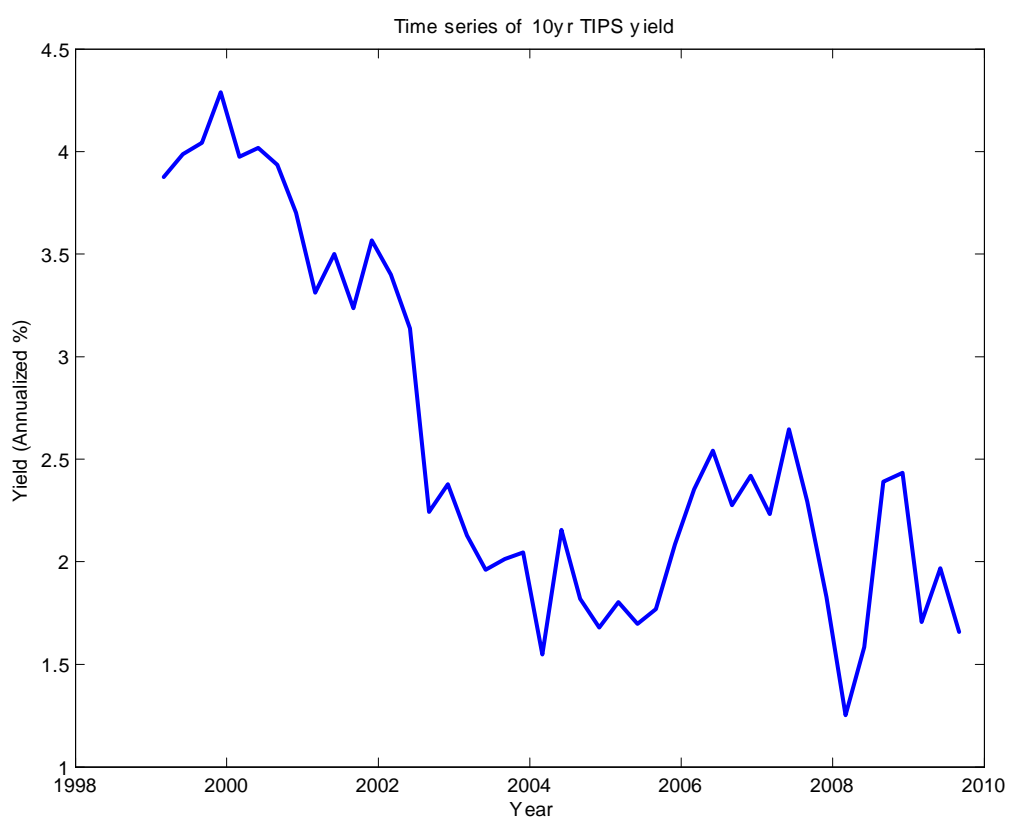

Figure 3: Time series of US 10-year inflation-indexed yields.
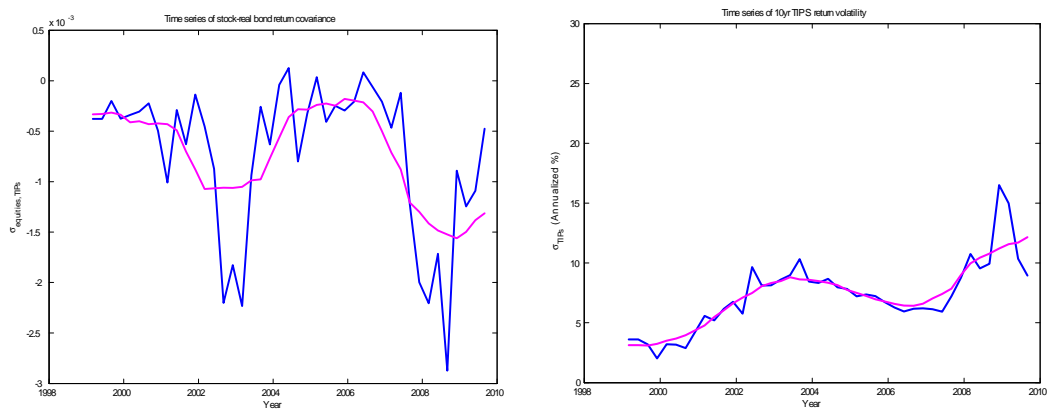

Figure 4: Time series of real bond second moments. The figure on the left shows the fitted value from a regression of the realized covariance between stock and real bond returns on lagged values of itself, the nominal short rate, and the yield spread. The figure on the right shows the fitted value from a regression of the realized variance of real bond returns on lagged values of itself, the nominal short rate, and the yield spread. The smoothed line in each figure is a 2-year equal-weighted moving average. 

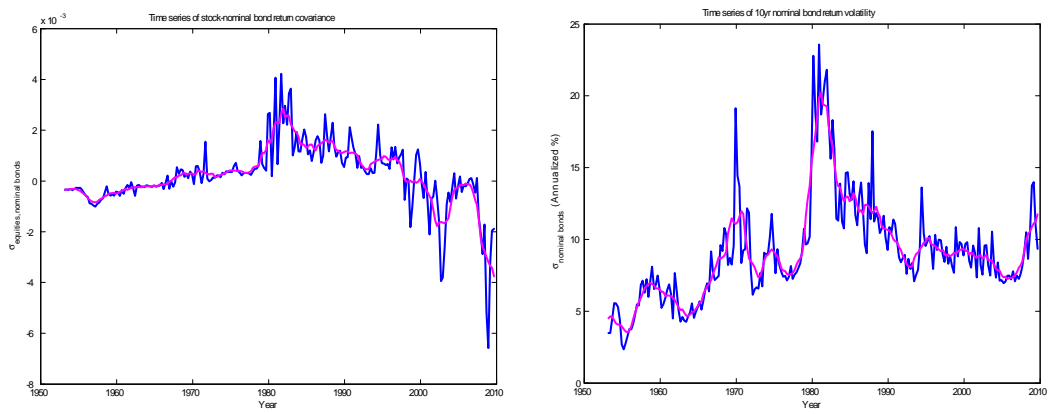

Figure 5: Time series of nominal bond second moments. The figure on the left shows the fitted value from a regression of the realized covariance between stock and nominal bond returns on lagged values of itself, the nominal short rate, and the yield spread. The figure on the right shows the fitted value from a regression of the realized variance of nominal bond returns on lagged values of itself, the nominal short rate, and the yield spread. The smoothed line in each figure is a 2-year equal-weighted moving average.

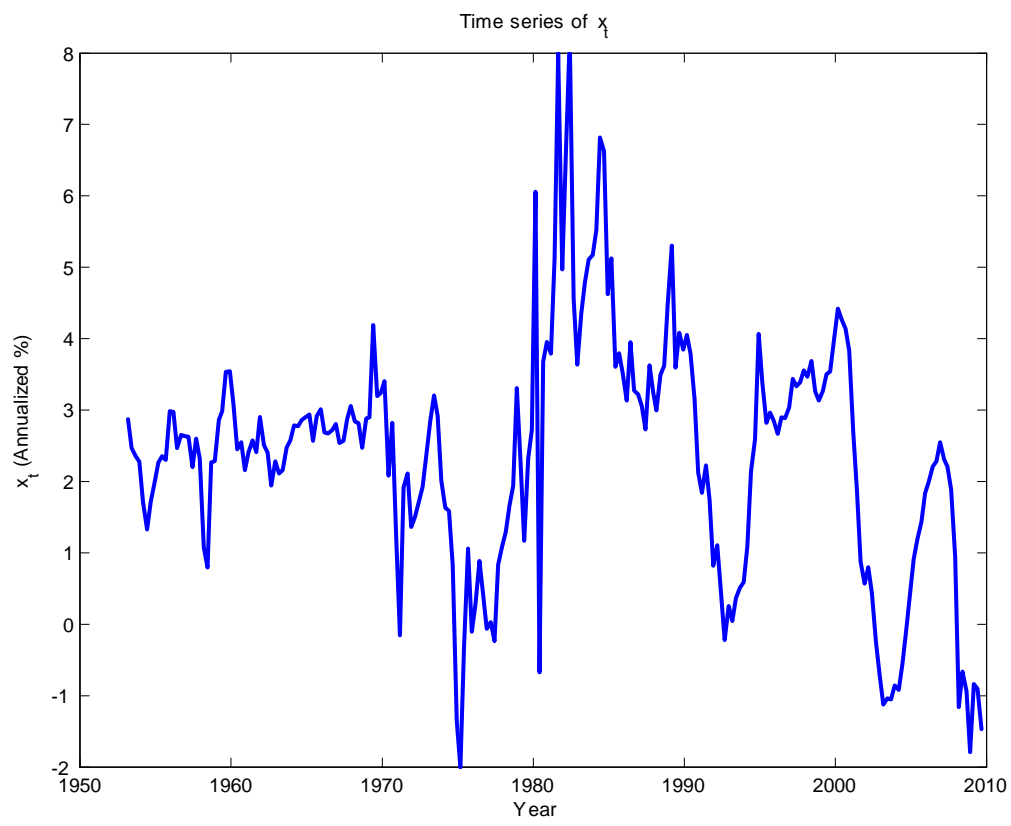

Figure 6: Estimated time series of the real rate. The figure plots the estimated time series of $x_{t}$, the real interest rate. 

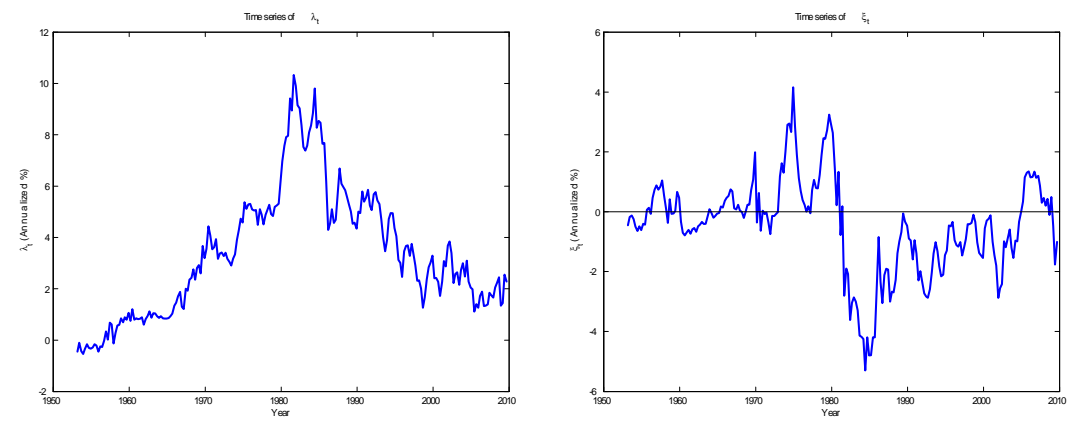

Figure 7: Estimated time series of permanent and transitory components of expected inflation. The figure on the left plots the estimated time series of $\lambda_{t}$, the permanent component of expected inflation. The figure on the right plots the estimated time series of $\xi_{t}$, the temporary component of expected inflation.

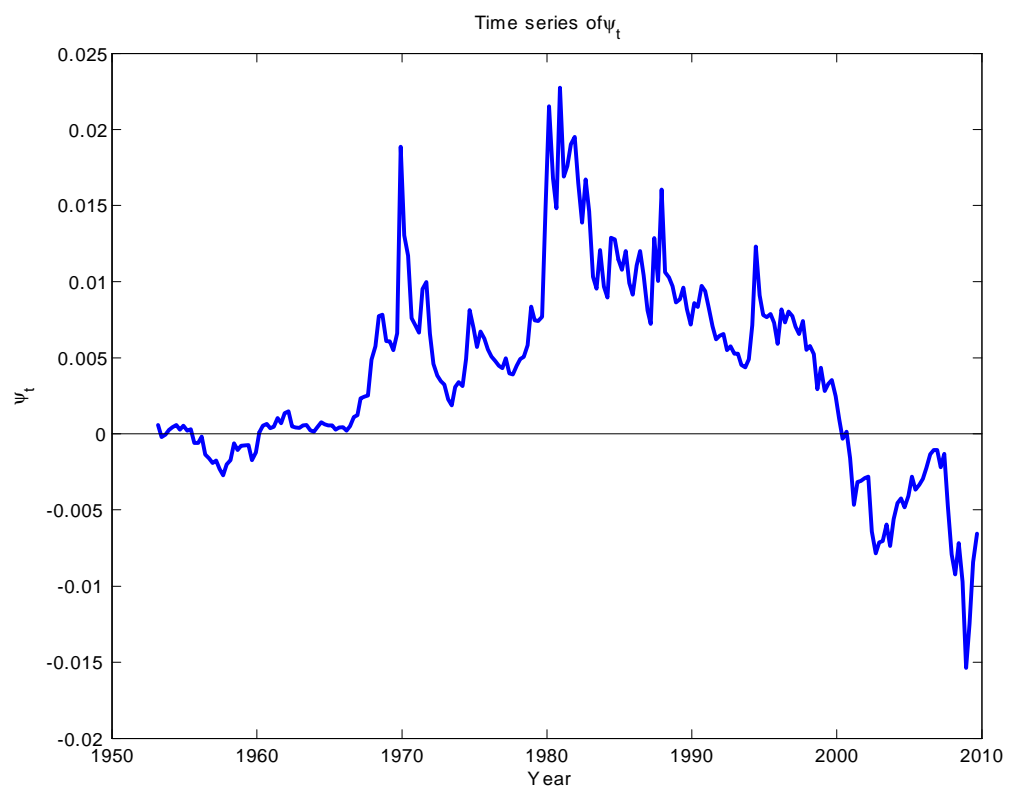

Figure 8: Estimated time series of $\psi_{t}$. 

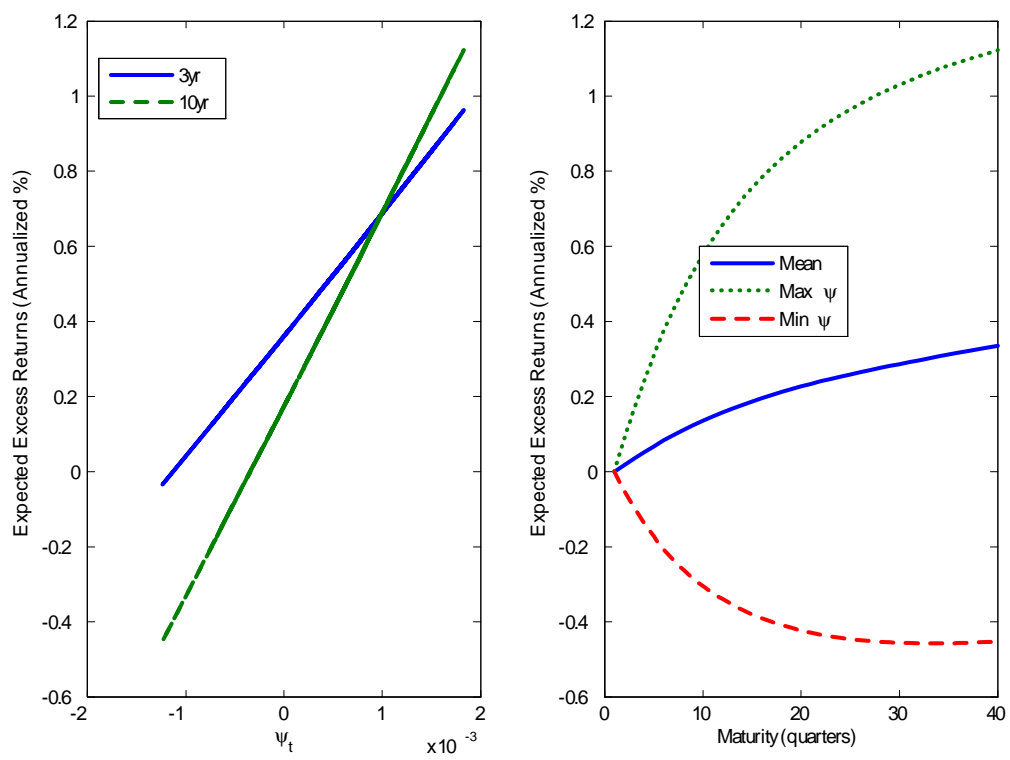

Figure 9: Responses of nominal expected excess returns to $\psi_{t}$. The left hand figure shows the expected excess returns on 3-year and 10-year nominal bonds over 3-month Treasury bills, as functions of $\psi_{t}$. The right hand figure shows the term structure of expected excess nominal bond returns as $\psi_{t}$ is varied between its sample minimum and maximum while all other state variables are held fixed at their sample means.

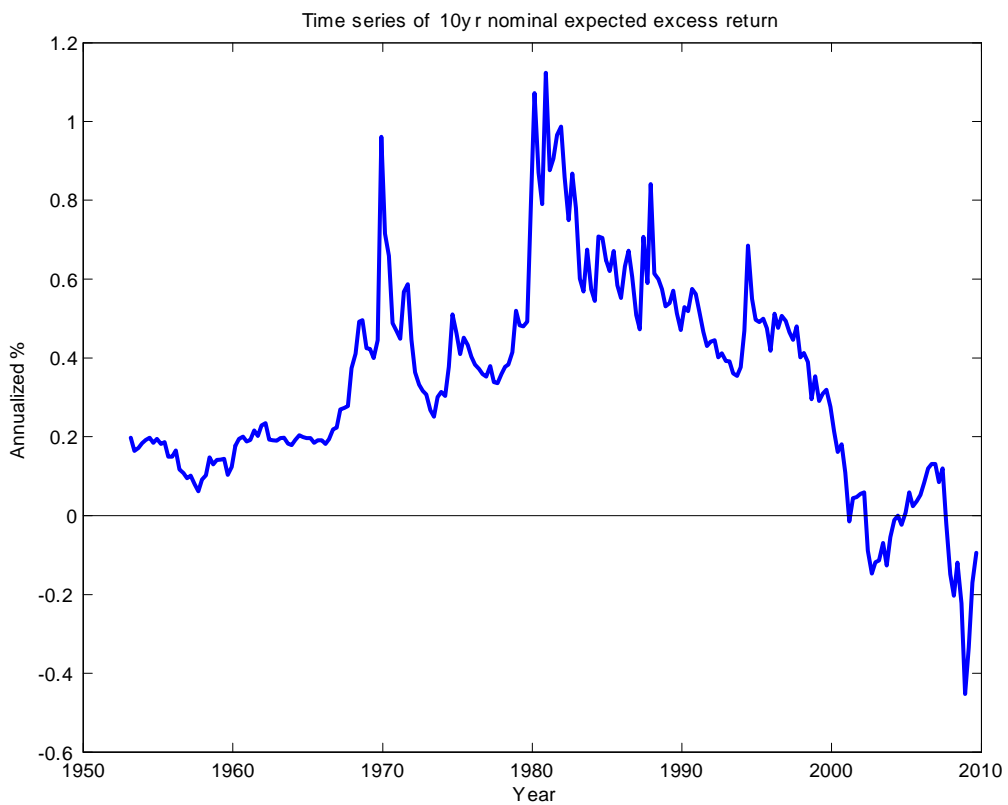

Figure 10: Estimated time series of expected excess returns for 10-year nominal bonds. 

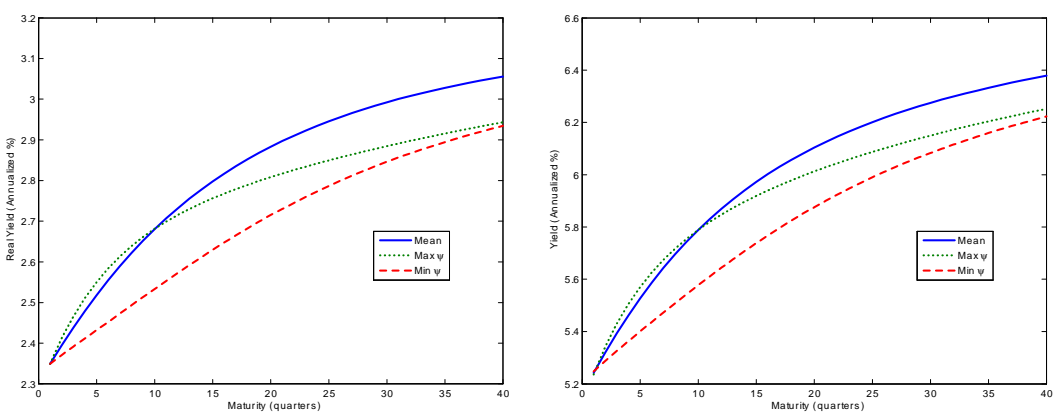

Figure 11: Responses of yield curves to $\psi_{t}$. The left hand figure shows the response of the real yield curve, and the right hand figure shows the response of the nominal yield curve, to $\psi_{t}$ as it is varied between its sample minima and maxima while all other state variables are held fixed at their sample means. 


\section{A Appendix}

A.1 Additional Empirical Results

A.1.1 Estimates of Covariances

\begin{tabular}{lcc}
\hline \hline \multicolumn{3}{c}{ Parameter Estimates } \\
\hline \hline Parameter & Estimate & Std Error \\
\hline$\sigma_{x \xi} \times 10^{-4}$ & 0.0021 & 53.0 \\
$\sigma_{x m} \times 10^{-2}$ & -7.18 & 4.41 \\
$\sigma_{X m} \times 10^{-7}$ & 0.968 & 2.25 \\
$\sigma_{x \pi} \times 10^{-2}$ & 0.007 & 18.1 \\
$\sigma_{\lambda m} \times 10^{-4}$ & -1.06 & 55.7 \\
$\sigma_{\Lambda m} \times 10^{-4}$ & -1.25 & 2.33 \\
$\sigma_{\xi m} \times 10^{-2}$ & -3.92 & 1.91 \\
$\sigma_{\xi \pi} \times 10^{-2}$ & -0.85 & 20.0 \\
$\sigma_{\psi m} \times 10^{-3}$ & -1.80 & 1.00 \\
$\sigma_{m \pi} \times 10^{-2}$ & 0.95 & 10.7 \\
\hline
\end{tabular}




\section{A.1.2 Results for Model Constraining $\sigma_{m}=.25$}

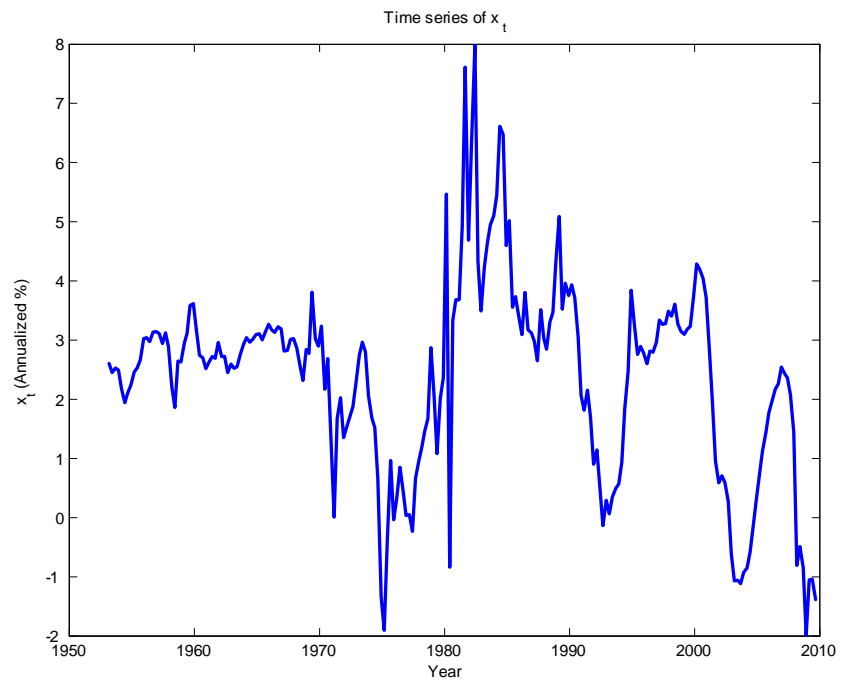

Figure 1: Estimated time series of the real rate. The figure plots the estimated time series of $x_{t}$, the real interest rate.
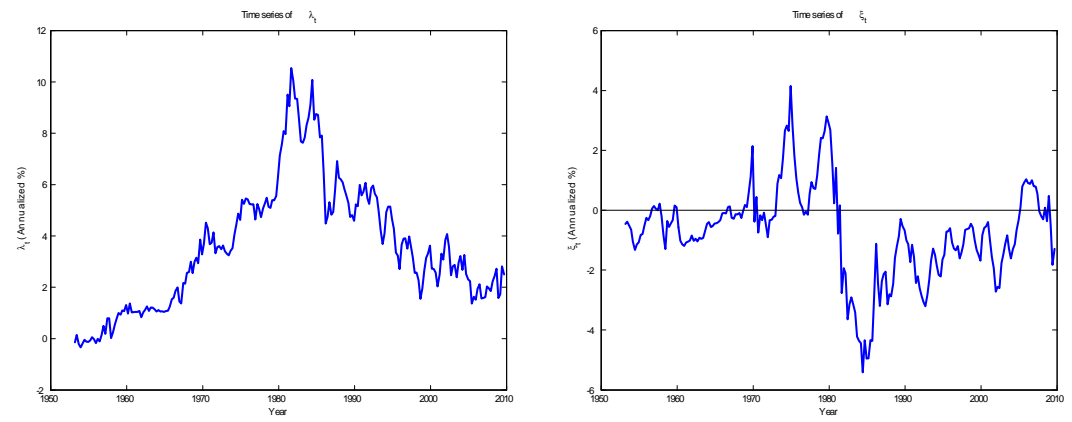

Figure 2: Estimated time series of permanent and transitory components of expected inflation. The figure on the left plots the estimated time series of $\lambda_{t}$, the permanent component of expected inflation. The figure on the right plots the estimated time series of $\xi_{t}$, the temporary component of expected inflation. 


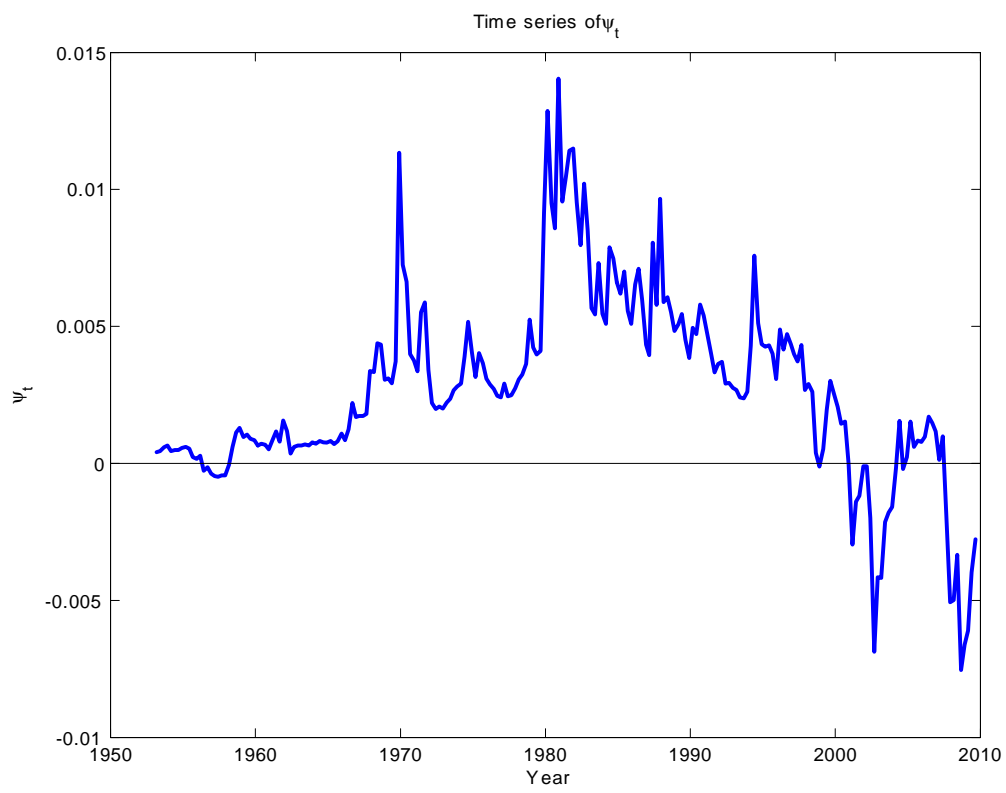

Figure 3: Estimated time series of $\psi_{t}$. 

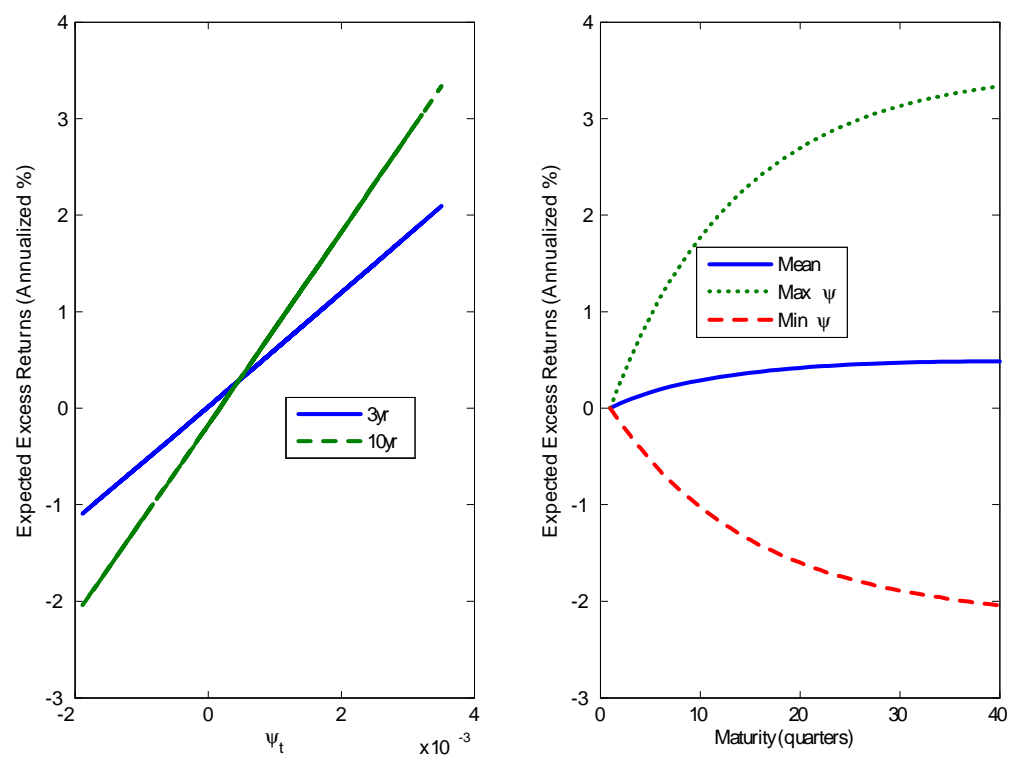

Figure 4: Responses of nominal expected excess returns to $\psi_{t}$. The left hand figure shows the expected excess returns on 3-year and 10-year nominal bonds over 3-month Treasury bills, as functions of $\psi_{t}$. The right hand figure shows the term structure of expected excess nominal bond returns as $\psi_{t}$ is varied between its sample minimum and maximum while all other state variables are held fixed at their sample means. 


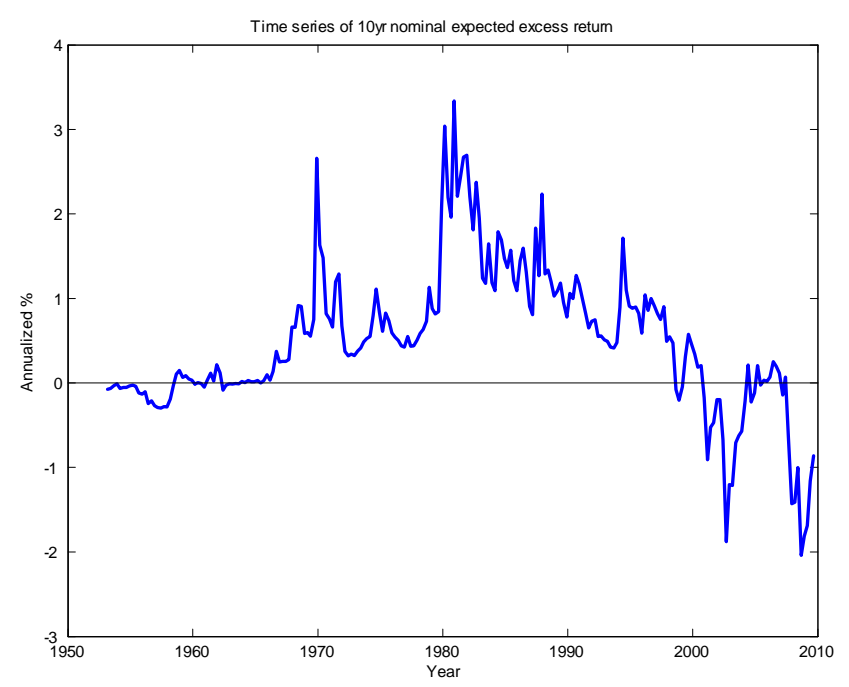

Figure 5: Estimated time series of expected excess returns for 10-year nominal bonds.
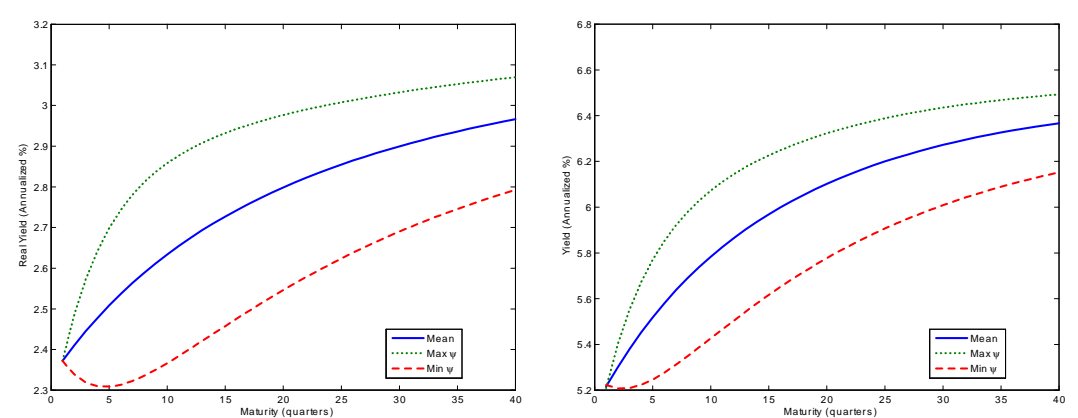

Figure 6: Responses of yield curves to $\psi_{t}$. The left hand figure shows the response of the real yield curve, and the right hand figure shows the response of the nominal yield curve, to $\psi_{t}$ as it is varied between its sample minima and maxima while all other state variables are held fixed at their sample means. 
Table 1: Parameter estimates.

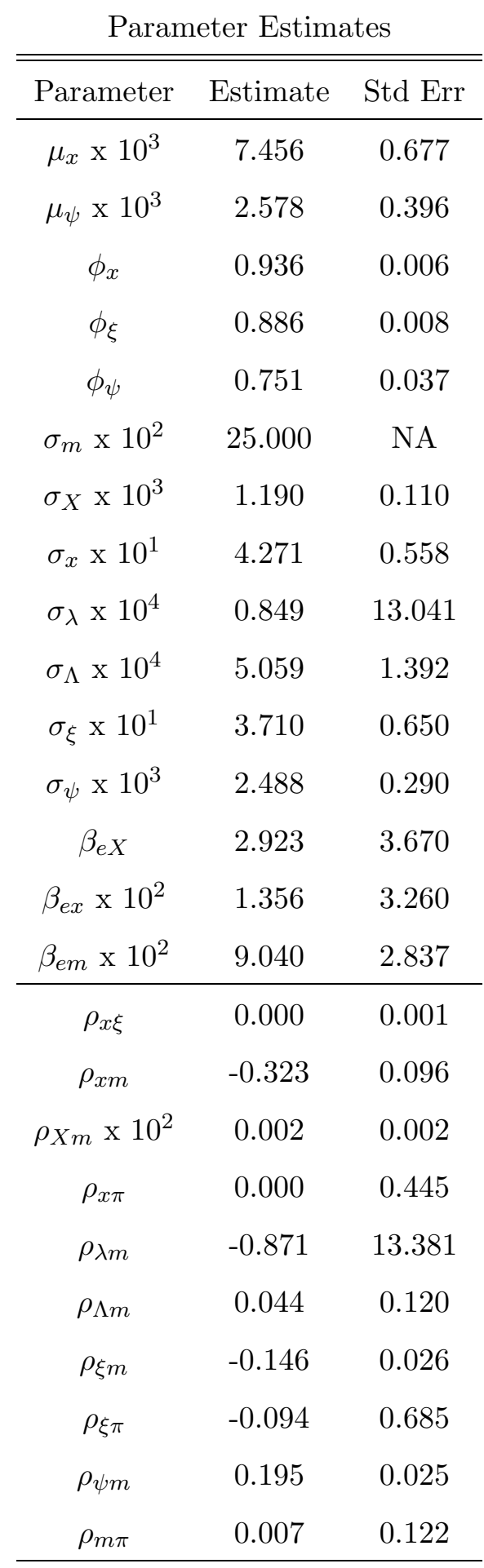


Table 2: Sample and Implied Moments. Yield spreads (YS) are calculated over the 3mo yield. Realized excess returns (RXR) are calculated over a 3mo holding period, in excess of the 1yr yield. Units are annualized percentage points. Simulation columns report means across 1000 replications, each of which simulates a time-series of 250 quarters. The $\sigma(\widehat{C P})$ row reports the standard deviation of the fitted values from a Cochrane-Piazzesi style regression of RXR on the 1-, 3-, and 5-yr forward rates at the beginning of the holding period. The $\sigma(\widehat{C S})$ row reports the standard deviation of the fitted values from a Campbell-Shiller style regression of RXR on the same-maturity YS at the beginning of the holding period. In the rightmost column we report the fraction of simulation runs where the simulated value exceeds the data value. ${ }^{\dagger}$ Data moments for the $10 \mathrm{yr}$ return require 117 mo yields. We interpolate the $117 \mathrm{mo}$ yield linearly between the $5 \mathrm{yr}$ and the $10 \mathrm{yr} \ddagger$ TIPS entries refer to the $10 \mathrm{yr}$ spliced TIPS yield. We have this data 1/1999-9/2009.

\begin{tabular}{cccc}
\hline \hline \multicolumn{4}{c}{ Sample and Implied Moments } \\
\hline \hline Moment & Actual Data & Model & Above \\
\hline 3yr YS mean & 0.62 & 0.57 & 0.43 \\
10yr YS mean & 1.15 & 0.94 & 0.37 \\
3yr YS stdev & 0.45 & 0.44 & 0.40 \\
10yr YS stdev & 0.70 & 0.89 & 0.89 \\
3yr RXR mean & 1.17 & 1.10 & 0.43 \\
10yr RXR mean & 2.21 & 1.64 & 0.31 \\
3yr RXR stdev & 4.37 & 4.27 & 0.38 \\
10yr RXR stdev & 11.16 & 8.03 & 0.00 \\
10yr TIPS yield mean & $2.58^{\ddagger}$ & 3.21 & 0.98 \\
10yr TIPS YS mean & & 0.15 & \\
10yr TIPS RXR mean & & 0.38 & \\
10yr TIPS RXR stdev & & 6.32 & \\
\hline
\end{tabular}

\begin{tabular}{cccc}
\hline \hline \multicolumn{4}{c}{ Predictive Regressions } \\
\hline \hline Moment & Actual Data & Model & Above \\
\hline 3yr EXR stdev & & 0.25 & \\
10yr EXR stdev & & 0.42 & \\
10yr TIPS EXR stdev & & 0.34 & \\
\hline 3yr RXR $\sigma(\widehat{C S})$ & 1.04 & 0.24 & 0.00 \\
10yr RXR $\sigma(\widehat{C S})$ & $2.51^{\dagger}$ & 0.44 & 0.00 \\
10yr TIPS RXR $\sigma(\widehat{C S})$ & & 0.39 & \\
\hline 3yr RXR $\sigma(\widehat{C P})$ & 0.79 & 0.53 & 0.14
\end{tabular}




\section{A.1.3 Results for Freely Estimated Model}

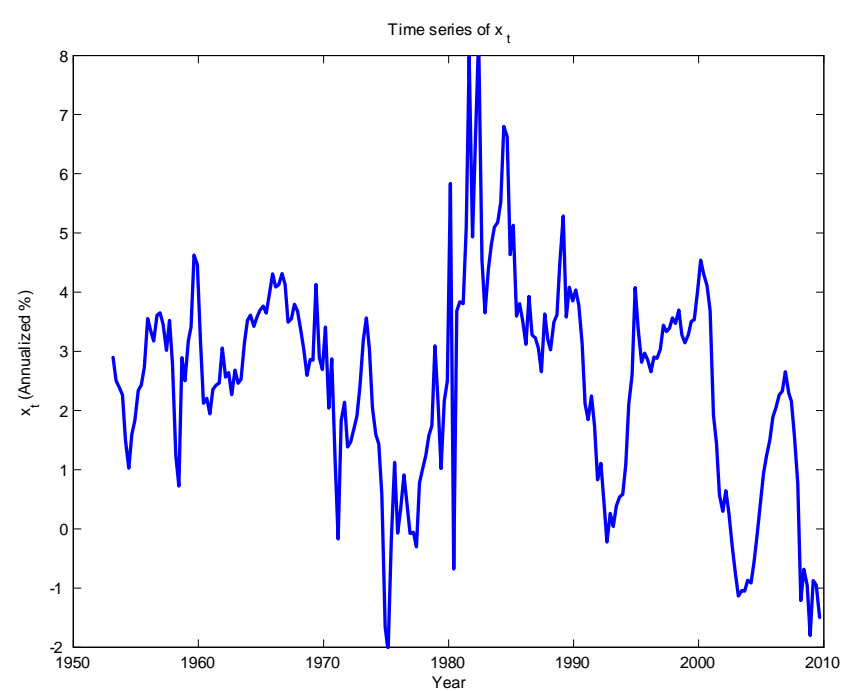

Figure 7: Estimated time series of the real rate. The figure plots the estimated time series of $x_{t}$, the real interest rate.
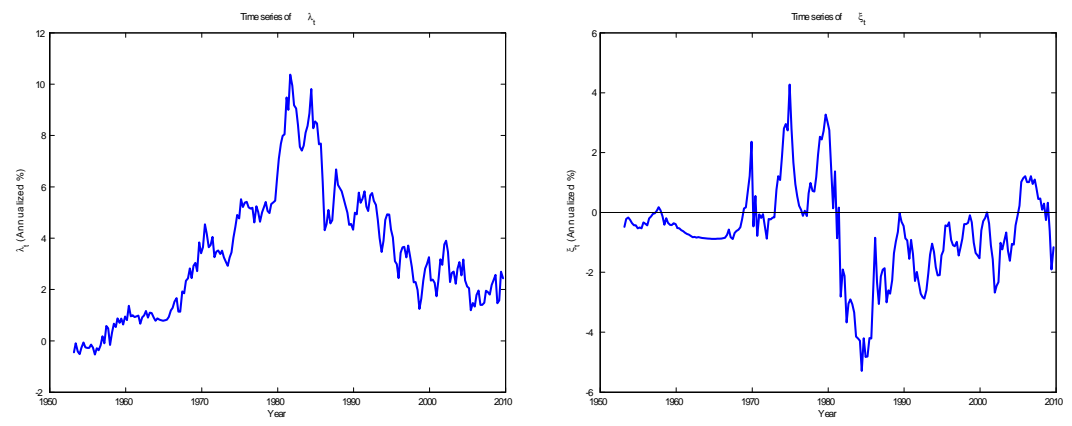

Figure 8: Estimated time series of permanent and transitory components of expected inflation. The figure on the left plots the estimated time series of $\lambda_{t}$, the permanent component of expected inflation. The figure on the right plots the estimated time series of $\xi_{t}$, the temporary component of expected inflation. 


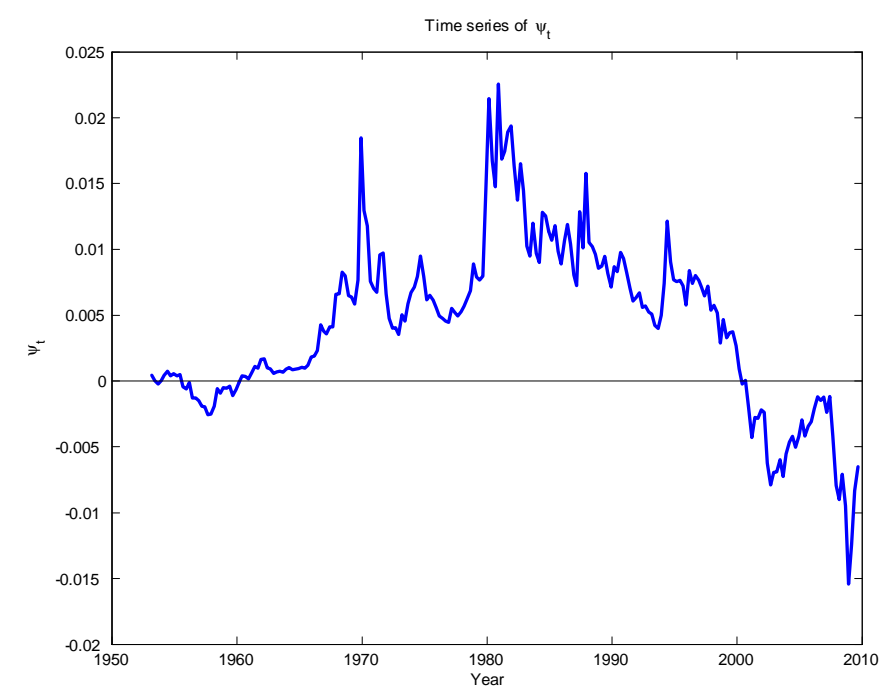

Figure 9: Estimated time series of $\psi_{t}$. 

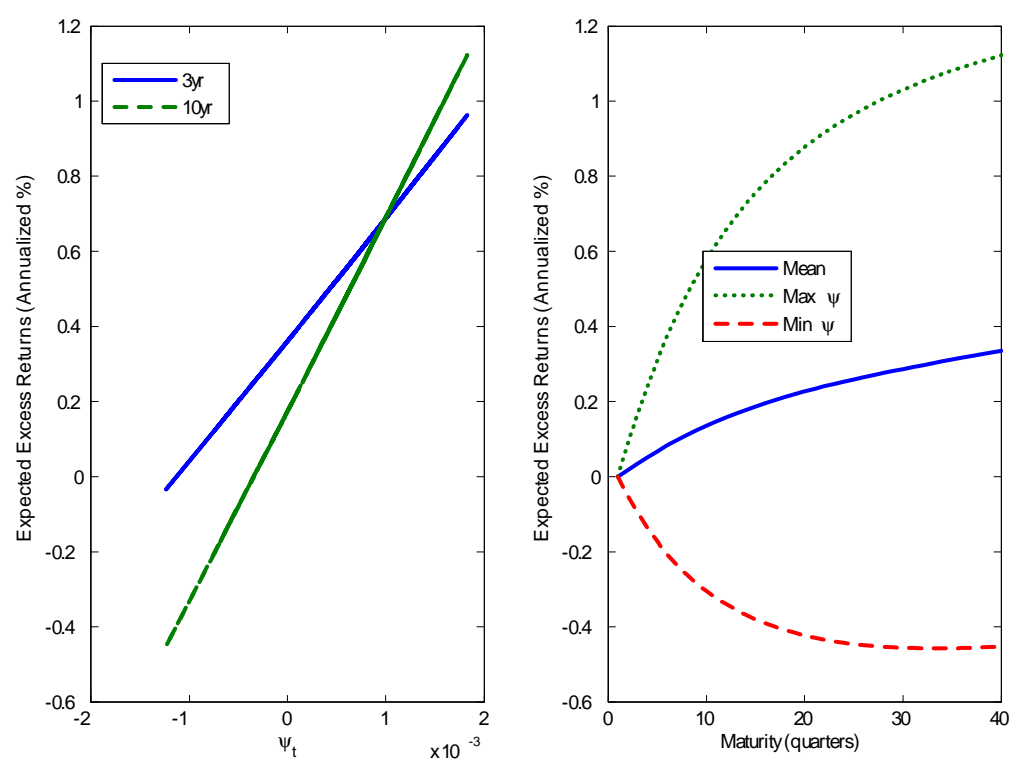

Figure 10: Responses of nominal expected excess returns to $\psi_{t}$. The left hand figure shows the expected excess returns on 3-year and 10-year nominal bonds over 3-month Treasury bills, as functions of $\psi_{t}$. The right hand figure shows the term structure of expected excess nominal bond returns as $\psi_{t}$ is varied between its sample minimum and maximum while all other state variables are held fixed at their sample means. 


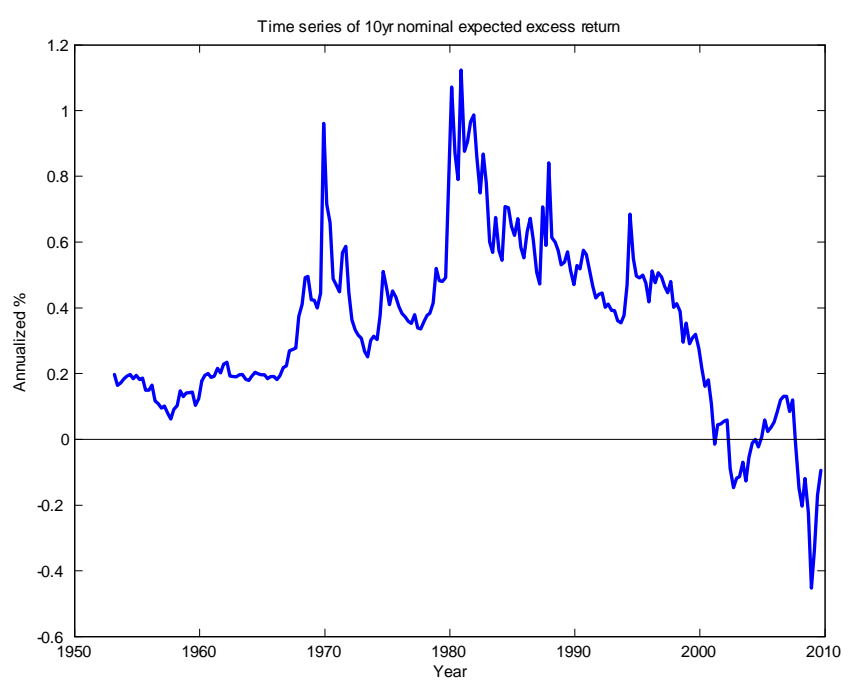

Figure 11: Estimated time series of expected excess returns for 10-year nominal bonds.
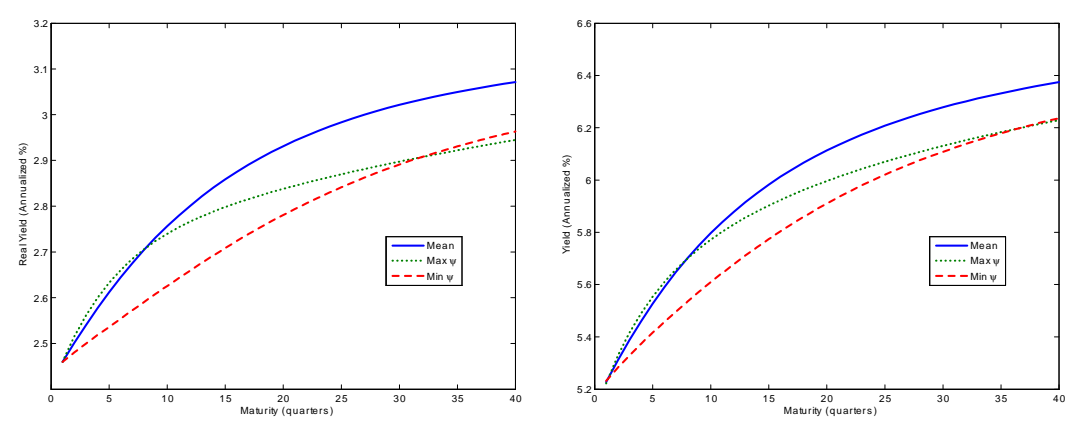

Figure 12: Responses of yield curves to $\psi_{t}$. The left hand figure shows the response of the real yield curve, and the right hand figure shows the response of the nominal yield curve, to $\psi_{t}$ as it is varied between its sample minima and maxima while all other state variables are held fixed at their sample means. 
Table 1: Parameter estimates.

\begin{tabular}{|c|c|}
\hline \multicolumn{2}{|c|}{ Parameter Estimates } \\
\hline Parameter & Estimate \\
\hline$\mu_{x} \times 10^{3}$ & 9.217 \\
\hline$\mu_{\psi} \times 10^{3}$ & 4.815 \\
\hline$\phi_{x}$ & 0.939 \\
\hline$\phi_{\xi}$ & 0.880 \\
\hline$\phi_{\psi}$ & 0.847 \\
\hline$\sigma_{m} \times 10^{2}$ & 8.019 \\
\hline$\sigma_{X} \times 10^{3}$ & 1.319 \\
\hline$\sigma_{x} \times 10^{1}$ & 2.919 \\
\hline$\sigma_{\lambda} \times 10^{4}$ & 1.443 \\
\hline$\sigma_{\Lambda} \times 10^{4}$ & 2.776 \\
\hline$\sigma_{\xi} \times 10^{1}$ & 2.415 \\
\hline$\sigma_{\psi} \times 10^{3}$ & 5.058 \\
\hline$\beta_{e X}$ & 1.133 \\
\hline$\beta_{e x} \times 10^{2}$ & 0.548 \\
\hline$\beta_{e m} \times 10^{2}$ & 9.538 \\
\hline$\rho_{x \xi}$ & 0.000 \\
\hline$\rho_{x m}$ & -0.246 \\
\hline$\rho_{X m} \times 10^{2}$ & 0.007 \\
\hline$\rho_{x \pi}$ & 0.000 \\
\hline$\rho_{\lambda m}$ & -0.732 \\
\hline$\rho_{\Lambda m}$ & -0.451 \\
\hline$\rho_{\xi m}$ & -0.163 \\
\hline$\rho_{\xi \pi}$ & -0.035 \\
\hline$\rho_{\psi m}$ & -0.347 \\
\hline$\rho_{m \pi}$ & 0.009 \\
\hline
\end{tabular}


Table 2: Sample and Implied Moments. Yield spreads (YS) are calculated over the 3mo yield. Realized excess returns (RXR) are calculated over a 3mo holding period, in excess of the 1yr yield. Units are annualized percentage points. Simulation columns report means across 1000 replications, each of which simulates a time-series of 250 quarters. The $\sigma(\widehat{C P})$ row reports the standard deviation of the fitted values from a Cochrane-Piazzesi style regression of RXR on the 1-, 3-, and 5-yr forward rates at the beginning of the holding period. The $\sigma(\widehat{C S})$ row reports the standard deviation of the fitted values from a Campbell-Shiller style regression of RXR on the same-maturity YS at the beginning of the holding period. In the rightmost column we report the fraction of simulation runs where the simulated value exceeds the data value. ${ }^{\dagger}$ Data moments for the $10 \mathrm{yr}$ return require 117 mo yields. We interpolate the $117 \mathrm{mo}$ yield linearly between the $5 \mathrm{yr}$ and the $10 \mathrm{yr} \ddagger$ TIPS entries refer to the $10 \mathrm{yr}$ spliced TIPS yield. We have this data 1/1999-9/2009.

\begin{tabular}{cccc}
\hline \hline \multicolumn{4}{c}{ Sample and Implied Moments } \\
\hline \hline Moment & Actual Data & Model & Above \\
\hline 3yr YS mean & 0.62 & 0.42 & 0.31 \\
10yr YS mean & 1.15 & 0.57 & 0.27 \\
3yr YS stdev & 0.45 & 0.67 & 0.96 \\
10yr YS stdev & 0.70 & 1.36 & 1.00 \\
3yr RXR mean & 1.17 & 0.91 & 0.38 \\
10yr RXR mean & 2.21 & 1.34 & 0.30 \\
3yr RXR stdev & 4.37 & 6.39 & 1.00 \\
10yr RXR stdev & 11.16 & 11.17 & 0.47 \\
10yr TIPS yield mean & $2.58^{\ddagger}$ & 3.57 & 0.97 \\
10yr TIPS YS mean & & -0.24 & \\
10yr TIPS RXR mean & & 0.04 & \\
10yr TIPS RXR stdev & & 9.99 & \\
\hline
\end{tabular}

\begin{tabular}{cccc}
\hline \hline \multicolumn{4}{c}{ Predictive Regressions } \\
\hline \hline Moment & Actual Data & Model & Above \\
\hline 3yr EXR stdev & & 0.12 & \\
10yr EXR stdev & & 0.19 & \\
10yr TIPS EXR stdev & & 0.14 & \\
\hline 3yr RXR $\sigma(\widehat{C S})$ & 1.04 & 0.39 & 0.03 \\
10yr RXR $\sigma(\widehat{C S})$ & $2.51^{\dagger}$ & 0.68 & 0.00 \\
10yr TIPS RXR $\sigma(\widehat{C S})$ & & 0.66 & \\
\hline 3yr RXR $\sigma(\widehat{C P})$ & 0.79 & 0.82 & 0.49
\end{tabular}




\section{A.2 Derivations for the Full Model}

\section{A.2.1 State Variable Processes}

The state variables in the model follow the processes:

$$
\begin{aligned}
-m_{t+1}= & x_{t}+\frac{1}{2} z_{t}^{2} \sigma_{m}^{2}+z_{t} \varepsilon_{m, t+1} \\
x_{t+1}= & \mu_{x}\left(1-\phi_{x}\right)+\phi_{x} x_{t}+\psi_{t} \varepsilon_{x, t+1}+\varepsilon_{X, t+1} \\
z_{t+1}= & \mu_{z}\left(1-\phi_{z}\right)+\phi_{z} z_{t}+\varepsilon_{z, t+1} \\
\pi_{t+1} & =\lambda_{t}+\xi_{t}+\frac{1}{2} \psi_{t}^{2} \sigma_{\pi}^{2}+\psi_{t} \varepsilon_{\pi, t+1} \\
\lambda_{t+1} & =\lambda_{t}+\psi_{t} \varepsilon_{\lambda, t+1}+\varepsilon_{\Lambda, t+1} \\
\xi_{t+1} & =\phi_{\xi} \xi_{t}+\psi_{t} \varepsilon_{\xi, t+1} \\
\psi_{t+1} & =\mu_{\psi}\left(1-\phi_{\psi}\right)+\phi_{\psi} \psi_{t}+\varepsilon_{\psi, t+1}
\end{aligned}
$$

\section{A.2.2 Pricing Equations}

Real Term Structure The price of a single-period zero-coupon real bond satisfies

$$
P_{1, t}=E_{t}\left[\exp \left\{m_{t+1}\right\}\right]=-x_{t}-\frac{1}{2} z_{t}^{2} \sigma_{m}^{2}+\frac{1}{2} z_{t}^{2} \sigma_{m}^{2}=-x_{t}
$$

We conjecture that the price function is exponential affine in $x_{t}$ and $z_{t}$ with the form

$$
P_{n, t}=\exp \left\{A_{n}+B_{x, n} x_{t}+B_{z, n} z_{t}+B_{\psi, n} \psi_{t}+C_{z, n} z_{t}^{2}+C_{\psi, n} \psi_{t}^{2}+C_{z \psi, n} z_{t} \psi_{t}\right\} .
$$

The standard pricing equation implies

$$
\begin{aligned}
P_{n, t}= & E_{t}\left[\exp \left\{p_{n-1, t+1}+m_{t+1}\right\}\right]=E_{t}\left[\exp \left\{\begin{array}{r}
A_{n-1}+B_{x, n-1} x_{t+1}+B_{z, n-1} z_{t+1}+B_{\psi, n-1} \psi_{t+1}+C_{z, n-1} z_{t+1}^{2}+C_{\psi, n-1} \psi_{t+1}^{2} \\
+C_{z \psi, n-1} z_{t+1} \psi_{t+1}-x_{t}-\frac{1}{2} z_{t}^{2} \sigma_{m}^{2}-z_{t} \varepsilon_{m, t+1}
\end{array}\right\} \begin{array}{r}
(1) \\
=
\end{array}\right. \\
& \exp \left\{\begin{array}{r}
A_{n-1}+B_{x, n-1}\left(\left(1-\phi_{x}\right) \mu_{x}+\phi_{x} x_{t}\right)+B_{z, n-1}\left(\left(1-\phi_{z}\right) \mu_{z}+\phi_{z} z_{t}\right)+B_{\psi, n-1}\left(\left(1-\phi_{\psi}\right) \mu_{\psi}+\phi_{\psi} \psi_{t}\right)+C_{z, n-1}\left(\mu_{z}\left(1-\phi_{z}\right)+\phi_{z} z_{t}\right)^{2} \\
+C_{\psi, n-1}\left(\mu_{\psi}\left(1-\phi_{\psi}\right)+\phi_{\psi} \psi_{t}\right)^{2}+C_{z \psi, n-1}\left(\mu_{z}\left(1-\phi_{z}\right)+\phi_{z} z_{t}\right)\left(\mu_{\psi}\left(1-\phi_{\psi}\right)+\phi_{\psi} \psi_{t}\right)-x_{t}-\frac{1}{2} z_{t}^{2} \sigma_{m}^{2}
\end{array}\right\} \\
& \times E_{t}\left[\exp \left\{\mathbf{d}_{1}^{\prime} \boldsymbol{\omega}_{t+1}+\boldsymbol{\omega}_{t+1}^{\prime} \mathbf{D}_{2} \boldsymbol{\omega}_{t+1}\right\}\right]
\end{aligned}
$$


where $\boldsymbol{\omega}_{t+1}^{\prime}=\left(\varepsilon_{X, t+1}, \varepsilon_{m, t+1}, \varepsilon_{x, t+1}, \varepsilon_{z, t+1}, \varepsilon_{\psi, t+1}\right)^{\sim} N\left(0, \boldsymbol{\Sigma}_{\omega}\right)$

$$
\mathbf{d}_{1}=\left(\begin{array}{c}
B_{x, n-1} \\
-z_{t} \\
B_{x, n-1} \psi_{t} \\
B_{z, n-1}+2 C_{z, n-1}\left(\mu_{z}\left(1-\phi_{z}\right)+\phi_{z} z_{t}\right)+C_{z \psi, n-1}\left(\mu_{\psi}\left(1-\phi_{\psi}\right)+\phi_{\psi} \psi_{t}\right) \\
B_{\psi, n-1}+2 C_{\psi, n-1}\left(\mu_{\psi}\left(1-\phi_{\psi}\right)+\phi_{\psi} \psi_{t}\right)+C_{z \psi, n-1}\left(\mu_{z}\left(1-\phi_{z}\right)+\phi_{z} z_{t}\right)
\end{array}\right)
$$

$$
\mathbf{D}_{2}=\left(\begin{array}{cccc}
0 & \cdots & & 0 \\
\vdots & \ddots & & \\
& & & \\
& & C_{z, n-1} & \frac{1}{2} C_{z \psi, n-1} \\
0 & & \frac{1}{2} C_{z \psi, n-1} & C_{\psi, n-1}
\end{array}\right)
$$

Following Campbell, Chan, and Viceira (2003), we complete the square to calculate

$$
\begin{aligned}
E_{t}\left[\exp \left\{\mathbf{d}_{1}^{\prime} \boldsymbol{\omega}_{t+1}+\boldsymbol{\omega}_{t+1}^{\prime} \mathbf{D}_{2} \boldsymbol{\omega}_{t+1}\right\}\right] & =\frac{\left|\boldsymbol{\Sigma}_{\omega}\right|^{-1 / 2}}{\left|\boldsymbol{\Sigma}_{\omega}^{-1}-2 \mathbf{D}_{2}\right|^{1 / 2}} \exp \left\{\frac{1}{2} \mathbf{d}_{1}\left(\boldsymbol{\Sigma}_{\omega}^{-1}-2 \mathbf{D}_{2}\right)^{-1} \mathbf{d}_{1}^{\prime}\right\} \\
& =\exp \left\{-\frac{1}{2} \log \left|\boldsymbol{\Sigma}_{\omega}\right|+\frac{1}{2} \log |\mathbf{G}|+\frac{1}{2} \mathbf{d}_{1} \mathbf{G d}_{1}^{\prime}\right\}
\end{aligned}
$$

where $\mathbf{G}=\left(\boldsymbol{\Sigma}_{\omega}^{-1}-2 \mathbf{D}_{2}\right)^{-1}$. Let $g_{i j}$ be the $i j$-th element of $\mathbf{G}$. Then expanding and collecting terms gives

$$
p_{n, t}=\left[\begin{array}{c}
A_{n-1}+B_{x, n-1}\left(\left(1-\phi_{x}\right) \mu_{x}+\phi_{x} x_{t}\right)+B_{z, n-1}\left(\left(1-\phi_{z}\right) \mu_{z}+\phi_{z} z_{t}\right)+B_{\psi, n-1}\left(\left(1-\phi_{\psi}\right) \mu_{\psi}+\phi_{\psi} \psi_{t}\right) \\
+C_{z, n-1}\left(\mu_{z}\left(1-\phi_{z}\right)+\phi_{z} z_{t}\right)^{2}+C_{\psi, n-1}\left(\mu_{\psi}\left(1-\phi_{\psi}\right)+\phi_{\psi} \psi_{t}\right)^{2}+C_{z \psi, n-1}\left(\mu_{z}\left(1-\phi_{z}\right)+\phi_{z} z_{t}\right)\left(\mu_{\psi}\left(1-\phi_{\psi}\right)+\phi_{\psi} \psi_{t}\right) \\
-x_{t}-\frac{1}{2} z_{t}^{2} \sigma_{m}^{2}-\frac{1}{2} \log \left|\boldsymbol{\Sigma}_{\omega}\right|+\frac{1}{2} \log |\mathbf{G}|+\frac{1}{2} g_{11} B_{x, n-1}^{2}+\frac{1}{2} g_{22} z_{t}^{2}+\frac{1}{2} g_{33} B_{x, n-1}^{2} \psi_{t}^{2} \\
+\frac{1}{2} g_{44}\left(B_{z, n-1}+2 C_{z, n-1}\left(\mu_{z}\left(1-\phi_{z}\right)+\phi_{z} z_{t}\right)+C_{z \psi, n-1}\left(\mu_{\psi}\left(1-\phi_{\psi}\right)+\phi_{\psi} \psi_{t}\right)\right)^{2} \\
+\frac{1}{2} g_{55}\left(B_{\psi, n-1}+2 C_{\psi, n-1}\left(\mu_{\psi}\left(1-\phi_{\psi}\right)+\phi_{\psi} \psi_{t}\right)+C_{z \psi, n-1}\left(\mu_{z}\left(1-\phi_{z}\right)+\phi_{z} z_{t}\right)\right)^{2} \\
-g_{12} B_{x, n-1} z_{t}+g_{13} B_{x, n-1}^{2} \psi_{t}+g_{14} B_{x, n-1}\left(B_{z, n-1}+2 C_{z, n-1}\left(\mu_{z}\left(1-\phi_{z}\right)+\phi_{z} z_{t}\right)+C_{z \psi, n-1}\left(\mu_{\psi}\left(1-\phi_{\psi}\right)+\phi_{\psi} \psi_{t}\right)\right) \\
+g_{15} B_{x, n-1}\left(B_{\psi, n-1}+2 C_{\psi, n-1}\left(\mu_{\psi}\left(1-\phi_{\psi}\right)+\phi_{\psi} \psi_{t}\right)+C_{z \psi, n-1}\left(\mu_{z}\left(1-\phi_{z}\right)+\phi_{z} z_{t}\right)\right) \\
-g_{23} B_{x, n-1} z_{t} \psi_{t}-g_{24} z_{t}\left(B_{z, n-1}+2 C_{z, n-1}\left(\mu_{z}\left(1-\phi_{z}\right)+\phi_{z} z_{t}\right)+C_{z \psi, n-1}\left(\mu_{\psi}\left(1-\phi_{\psi}\right)+\phi_{\psi} \psi_{t}\right)\right) \\
-g_{25} z_{t}\left(B_{\psi, n-1}+2 C_{\psi, n-1}\left(\mu_{\psi}\left(1-\phi_{\psi}\right)+\phi_{\psi} \psi_{t}\right)+C_{z \psi, n-1}\left(\mu_{z}\left(1-\phi_{z}\right)+\phi_{z} z_{t}\right)\right) \\
+g_{34} B_{x, n-1} \psi_{t}\left(B_{z, n-1}+2 C_{z, n-1}\left(\mu_{z}\left(1-\phi_{z}\right)+\phi_{z} z_{t}\right)+C_{z \psi, n-1}\left(\mu_{\psi}\left(1-\phi_{\psi}\right)+\phi_{\psi} \psi_{t}\right)\right) \\
+g_{35} B_{x, n-1} \psi_{t}\left(B_{\psi, n-1}+2 C_{\psi, n-1}\left(\mu_{\psi}\left(1-\phi_{\psi}\right)+\phi_{\psi} \psi_{t}\right)+C_{z \psi, n-1}\left(\mu_{z}\left(1-\phi_{z}\right)+\phi_{z} z_{t}\right)\right) \\
+g_{45}\left(B_{z, n-1}+2 C_{z, n-1}\left(\mu_{z}\left(1-\phi_{z}\right)+\phi_{z} z_{t}\right)+C_{z \psi, n-1}\left(\mu_{\psi}\left(1-\phi_{\psi}\right)+\phi_{\psi} \psi_{t}\right)\right) \\
\times\left(B_{\psi, n-1}+2 C_{\psi, n-1}\left(\mu_{\psi}\left(1-\phi_{\psi}\right)+\phi_{\psi} \psi_{t}\right)+C_{z \psi, n-1}\left(\mu_{z}\left(1-\phi_{z}\right)+\phi_{z} z_{t}\right)\right)
\end{array}\right]
$$

Thus, equating coefficients across equation (1) yields 


$$
\begin{aligned}
& A_{n}=\left[\begin{array}{c}
A_{n-1}+B_{x, n-1}\left(1-\phi_{x}\right) \mu_{x}+B_{z, n-1}\left(1-\phi_{z}\right) \mu_{z}+B_{\psi, n-1}\left(1-\phi_{\psi}\right) \mu_{\psi} \\
+C_{z, n-1} \mu_{z}^{2}\left(1-\phi_{z}\right)^{2}+C_{\psi, n-1} \mu_{\psi}^{2}\left(1-\phi_{\psi}\right)^{2}+C_{z \psi, n-1} \mu_{z}\left(1-\phi_{z}\right) \mu_{\psi}\left(1-\phi_{\psi}\right) \\
-\frac{1}{2} \log \left|\boldsymbol{\Sigma}_{\omega}\right|+\frac{1}{2} \log |\mathbf{G}|+\frac{1}{2} g_{11} B_{x, n-1}^{2}+\frac{1}{2} g_{44}\left(B_{z, n-1}+2 C_{z, n-1} \mu_{z}\left(1-\phi_{z}\right)+C_{z \psi, n-1} \mu_{\psi}\left(1-\phi_{\psi}\right)\right)^{2} \\
+\frac{1}{2} g_{55}\left(B_{\psi, n-1}+2 C_{\psi, n-1} \mu_{\psi}\left(1-\phi_{\psi}\right)+C_{z \psi, n-1} \mu_{z}\left(1-\phi_{z}\right)\right)^{2} \\
+g_{14} B_{x, n-1}\left(B_{z, n-1}+2 C_{z, n-1} \mu_{z}\left(1-\phi_{z}\right)+C_{z \psi, n-1} \mu_{\psi}\left(1-\phi_{\psi}\right)\right)+g_{15} B_{x, n-1}\left(B_{\psi, n-1}+2 C_{\psi, n-1} \mu_{\psi}\left(1-\phi_{\psi}\right)+C_{z \psi, n-1} \mu_{z}\left(1-\phi_{z}\right)\right) \\
+g_{45}\left(B_{z, n-1}+2 C_{z, n-1} \mu_{z}\left(1-\phi_{z}\right)+C_{z \psi, n-1} \mu_{\psi}\left(1-\phi_{\psi}\right)\right)\left(B_{\psi, n-1}+2 C_{\psi, n-1} \mu_{\psi}\left(1-\phi_{\psi}\right)+C_{z \psi, n-1} \mu_{z}\left(1-\phi_{z}\right)\right)
\end{array}\right] \\
& B_{x, n}=B_{x, n-1} \phi_{x}-1 \\
& B_{z, n}=\left[\begin{array}{c}
B_{z, n-1} \phi_{z}+2 C_{z, n-1} \mu_{z}\left(1-\phi_{z}\right) \phi_{z}+C_{z \psi, n-1} \mu_{\psi}\left(1-\phi_{\psi}\right) \phi_{z}+2 g_{44}\left(B_{z, n-1}+2 C_{z, n-1} \mu_{z}\left(1-\phi_{z}\right)+C_{z \psi, n-1} \mu_{\psi}\left(1-\phi_{\psi}\right)\right) C_{z, n-1} \phi_{z} \\
+g_{55}\left(B_{\psi, n-1}+2 C_{\psi, n-1} \mu_{\psi}\left(1-\phi_{\psi}\right)+C_{z \psi, n-1} \mu_{z}\left(1-\phi_{z}\right)\right) C_{z \psi, n-1} \phi_{z}-g_{12} B_{x, n-1}+2 g_{14} B_{x, n-1} C_{z, n-1} \phi_{z}+g_{15} B_{x, n-1} C_{z \psi, n-1} \phi_{z} \\
-g_{24}\left(B_{z, n-1}+2 C_{z, n-1} \mu_{z}\left(1-\phi_{z}\right)+C_{z \psi, n-1} \mu_{\psi}\left(1-\phi_{\psi}\right)\right)-g_{25}\left(B_{\psi, n-1}+2 C_{\psi, n-1} \mu_{\psi}\left(1-\phi_{\psi}\right)+C_{z \psi, n-1} \mu_{z}\left(1-\phi_{z}\right)\right) \\
+g_{45}\left[\begin{array}{c}
2 C_{z, n-1}\left(B_{\psi, n-1}+2 C_{\psi, n-1} \mu_{\psi}\left(1-\phi_{\psi}\right)+C_{z \psi, n-1} \mu_{z}\left(1-\phi_{z}\right)\right) \\
+C_{z \psi, n-1}\left(B_{z, n-1}+2 C_{z, n-1} \mu_{z}\left(1-\phi_{z}\right)+C_{z \psi, n-1} \mu_{\psi}\left(1-\phi_{\psi}\right)\right)
\end{array}\right] \phi_{z}
\end{array}\right] \\
& B_{\psi, n}=\left[\begin{array}{c}
B_{\psi, n-1} \phi_{\psi}+2 C_{\psi, n-1} \mu_{\psi}\left(1-\phi_{\psi}\right) \phi_{\psi}+C_{z \psi, n-1} \mu_{z}\left(1-\phi_{z}\right) \phi_{\psi}+g_{13} B_{x, n-1}^{2}+g_{14} B_{x, n-1} C_{z \psi, n-1} \phi_{\psi}+2 g_{15} B_{x, n-1} C_{\psi, n-1} \phi_{\psi} \\
+g_{44}\left(B_{z, n-1}+2 C_{z, n-1} \mu_{z}\left(1-\phi_{z}\right)+C_{z \psi, n-1} \mu_{\psi}\left(1-\phi_{\psi}\right)\right) C_{z \psi, n-1} \phi_{\psi} \\
+2 g_{55}\left(B_{\psi, n-1}+2 C_{\psi, n-1} \mu_{\psi}\left(1-\phi_{\psi}\right)+C_{z \psi, n-1} \mu_{z}\left(1-\phi_{z}\right)\right) C_{\psi, n-1} \phi_{\psi} \\
+g_{34} B_{x, n-1}\left(B_{z, n-1}+2 C_{z, n-1} \mu_{z}\left(1-\phi_{z}\right)+C_{z \psi, n-1} \mu_{\psi}\left(1-\phi_{\psi}\right)\right)+g_{35} B_{x, n-1}\left(B_{\psi, n-1}+2 C_{\psi, n-1} \mu_{\psi}\left(1-\phi_{\psi}\right)+C_{z \psi, n-1} \mu_{z}\left(1-\phi_{z}\right)\right) \\
+g_{45}\left[\begin{array}{c}
2 C_{\psi, n-1}\left(B_{z, n-1}+2 C_{z, n-1} \mu_{z}\left(1-\phi_{z}\right)+C_{z \psi, n-1} \mu_{\psi}\left(1-\phi_{\psi}\right)\right) \\
+C_{z \psi, n-1}\left(B_{\psi, n-1}+2 C_{\psi, n-1} \mu_{\psi}\left(1-\phi_{\psi}\right)+C_{z \psi, n-1} \mu_{z}\left(1-\phi_{z}\right)\right)
\end{array}\right] \phi_{\psi}
\end{array}\right] \\
& C_{z, n}=\left[C_{z, n-1} \phi_{z}^{2}-\frac{1}{2} \sigma_{m}^{2}+\frac{1}{2} g_{22}+2 g_{44} C_{z, n-1}^{2} \phi_{z}^{2}+\frac{1}{2} g_{55} C_{z \psi, n-1}^{2} \phi_{z}^{2}-2 g_{24} C_{z, n-1} \phi_{z}-g_{25} C_{z \psi, n-1} \phi_{z}+2 g_{45} C_{z, n-1} C_{z \psi, n-1} \phi_{z}^{2}\right] \\
& C_{\psi, n}=\left[C_{\psi, n-1} \phi_{\psi}^{2}+\frac{1}{2} g_{33} B_{x, n-1}^{2}+\frac{1}{2} g_{44} C_{z \psi, n-1}^{2} \phi_{\psi}^{2}+2 g_{55} C_{\psi, n-1}^{2} \phi_{\psi}^{2}+g_{34} B_{x, n-1} C_{z \psi, n-1} \phi_{\psi}+2 g_{35} B_{x, n-1} C_{\psi, n-1} \phi_{\psi}+2 g_{45} C_{\psi, n-1} C_{z \psi, n-1} \phi_{\psi}^{2}\right] \\
& C_{z \psi, n}=\left[\begin{array}{c}
C_{z \psi, n-1} \phi_{z} \phi_{\psi}+2 g_{44} C_{z, n-1} C_{z \psi, n-1} \phi_{z} \phi_{\psi}+2 g_{55} C_{\psi, n-1} C_{z \psi, n-1} \phi_{z} \phi_{\psi}-g_{23} B_{x, n-1}-g_{24} C_{z \psi, n-1} \phi_{\psi}-2 g_{25} C_{\psi, n-1} \phi_{\psi} \\
+2 g_{34} B_{x, n-1} C_{z, n-1} \phi_{z}+g_{35} B_{x, n-1} C_{z \psi, n-1} \phi_{z}+g_{45} C_{z \psi, n-1}^{2} \phi_{\psi} \phi_{z}
\end{array}\right]
\end{aligned}
$$

Nominal Term Structure The price of a single-period zero-coupon nominal bond satisfies

$$
P_{1, t}^{\$}=E_{t}\left[\exp \left\{m_{t+1}-\pi_{t+1}\right\}\right]=\exp \left\{-x_{t}-\lambda_{t}-\xi_{t}+z_{t} \psi_{t} \sigma_{m \pi}\right\}
$$

since $z_{t} \varepsilon_{m, t+1}$ and $\psi_{t} \varepsilon_{\pi, t+1}$ are jointly conditional normal.

We now guess that the price function is exponential linear-quadratic in the state variables with the following form:

$$
P_{n, t}^{\$}=\exp \left\{A_{n}^{\$}+B_{x, n}^{\$} x_{t}+B_{z, n}^{\$} z_{t}+B_{\lambda, n}^{\$} \lambda_{t}+B_{\xi, n}^{\$} \xi_{t}+B_{\psi, n}^{\$} \psi_{t}+C_{z, n}^{\$} z_{t}^{2}+C_{\psi, n}^{\$} \psi_{t}^{2}+C_{z \psi, n}^{\$} z_{t} \psi_{t}\right\}
$$


The standard pricing equation then implies

$$
\begin{aligned}
& P_{n, t}^{\$}=E_{t}\left[\exp \left\{p_{n-1, t+1}^{\$}+m_{t+1}-\pi_{t+1}\right\}\right] \\
& =E_{t}\left[\exp \left\{\begin{array}{c}
A_{n-1}^{\$}+B_{x, n-1}^{\$} x_{t+1}+B_{z, n-1}^{\$} z_{t+1}+B_{\lambda, n-1}^{\$} \lambda_{t+1}+B_{\xi, n-1}^{\$} \xi_{t+1}+B_{\psi, n-1}^{\$} \psi_{t+1} \\
+C_{z, n-1}^{\$} z_{t+1}^{2}+C_{\psi, n-1}^{\$} \psi_{t+1}^{2}+C_{z \psi, n-1}^{\$} z_{t+1} \psi_{t+1} \\
-x_{t}-\frac{1}{2} z_{t}^{2} \sigma_{m}^{2}-z_{t} \varepsilon_{m, t+1}-\lambda_{t}-\xi_{t}-\frac{1}{2} \psi_{t}^{2} \sigma_{\pi}^{2}-\psi_{t} \varepsilon_{\pi, t+1}
\end{array}\right\}\right] \\
& =\exp \left\{\begin{array}{c}
A_{n-1}^{\Phi}+B_{x, n-1}^{\$}\left(\mu_{x}\left(1-\phi_{x}\right)+\phi_{x} x_{t}\right)+B_{z, n-1}^{\$}\left(\mu_{z}\left(1-\phi_{z}\right)+\phi_{z} z_{t}\right)+B_{\lambda, n-1}^{\Phi}\left(\mu_{\lambda}+\lambda_{t}\right)+B_{\xi, n-1}^{\$} \phi_{\xi} \xi_{t}+B_{\psi, n-1}^{\Phi}\left(\mu_{\psi}\left(1-\phi_{\psi}\right)+\phi_{\psi} \psi_{t}\right) \\
+C_{z, n-1}^{\Phi}\left(\mu_{z}\left(1-\phi_{z}\right)+\phi_{z} z_{t}\right)^{2}+C_{\psi, n-1}^{\Phi}\left(\mu_{\psi}\left(1-\phi_{\psi}\right)+\phi_{\psi} \psi_{t}\right)^{2}+C_{z \psi, n-1}^{\Phi}\left(\mu_{z}\left(1-\phi_{z}\right)+\phi_{z} z_{t}\right)\left(\mu_{\psi}\left(1-\phi_{\psi}\right)+\phi_{\psi} \psi_{t}\right) \\
-x_{t}-\frac{1}{2} z_{t}^{2} \sigma_{m}^{2}-\lambda_{t}-\xi_{t}-\frac{1}{2} \psi_{t}^{2} \sigma_{\pi}^{2}
\end{array}\right\} \\
& \times E_{t}\left[\exp \left\{\mathbf{d}_{1}^{\$ \prime} \boldsymbol{\omega}_{t+1}^{\$}+\boldsymbol{\omega}_{t+1}^{\$ \prime} \mathbf{D}_{2}^{\$} \boldsymbol{\omega}_{t+1}^{\$}\right\}\right]
\end{aligned}
$$

where $\boldsymbol{\omega}_{t+1}^{\$ \prime}=\left(\varepsilon_{X, t+1}, \varepsilon_{\Lambda, t+1}, \varepsilon_{\lambda, t+1}, \varepsilon_{m, t+1}, \varepsilon_{\pi, t+1}, \varepsilon_{x, t+1}, \varepsilon_{\xi, t+1}, \varepsilon_{z, t+1}, \varepsilon_{\psi, t+1}\right) \sim N\left(0, \boldsymbol{\Sigma}_{\omega}^{\$}\right)$,

$$
\mathbf{d}_{1}^{\$}=\left(\begin{array}{c}
B_{x, n-1}^{\$} \\
B_{\lambda, n-1}^{\$} \\
B_{\lambda, n-1}^{\$} \psi_{t} \\
-z_{t} \\
-\psi_{t} \\
B_{x, n-1}^{\$} \psi_{t} \\
B_{\xi, n-1}^{\$} \psi_{t} \\
B_{z, n-1}^{\$}+2 C_{z, n-1}^{\$}\left(\mu_{z}\left(1-\phi_{z}\right)+\phi_{z} z_{t}\right)+C_{z \psi, n-1}^{\$}\left(\mu_{\psi}\left(1-\phi_{\psi}\right)+\phi_{\psi} \psi_{t}\right) \\
B_{\psi, n-1}^{\$}+2 C_{\psi, n-1}^{\Phi}\left(\mu_{\psi}\left(1-\phi_{\psi}\right)+\phi_{\psi} \psi_{t}\right)+C_{z \psi, n-1}^{\$}\left(\mu_{z}\left(1-\phi_{z}\right)+\phi_{z} z_{t}\right)
\end{array}\right)
$$

$$
\mathbf{D}_{2}^{\$}=\left(\begin{array}{cccc}
0 & \cdots & & 0 \\
& & & \vdots \\
\vdots & \ddots & & \\
& & C_{z, n-1}^{\$} & \frac{1}{2} C_{z \psi, n-1}^{\$} \\
0 & \cdots & \frac{1}{2} C_{z \psi, n-1}^{\Phi} & C_{\psi, n-1}^{\Phi}
\end{array}\right)
$$

Following Campbell, Chan, and Viceira (2003), we complete the square to calculate

$$
E_{t}\left[\exp \left\{\mathbf{d}_{1}^{\$ \prime} \boldsymbol{\omega}_{t+1}^{\$}+\boldsymbol{\omega}_{t+1}^{\$ \prime} \mathbf{D}_{2}^{\$} \boldsymbol{\omega}_{t+1}^{\$}\right\}\right]=\exp \left\{-\frac{1}{2} \log \left|\boldsymbol{\Sigma}_{\omega}^{\$}\right|+\frac{1}{2} \log \left|\mathbf{G}^{\$}\right|+\frac{1}{2} \mathbf{d}_{1}^{\$} \mathbf{G}^{\$} \mathbf{d}_{1}^{\$ \prime}\right\}
$$


where $\mathbf{G}^{\$}=\left(\boldsymbol{\Sigma}_{\omega}^{\$-1}-2 \mathbf{D}_{2}^{\$}\right)^{-1}$. Let $g_{i j}^{\$}$ be the $i j$-th element of $\mathbf{G}$. Then expanding and collecting terms gives $g^{\$}$

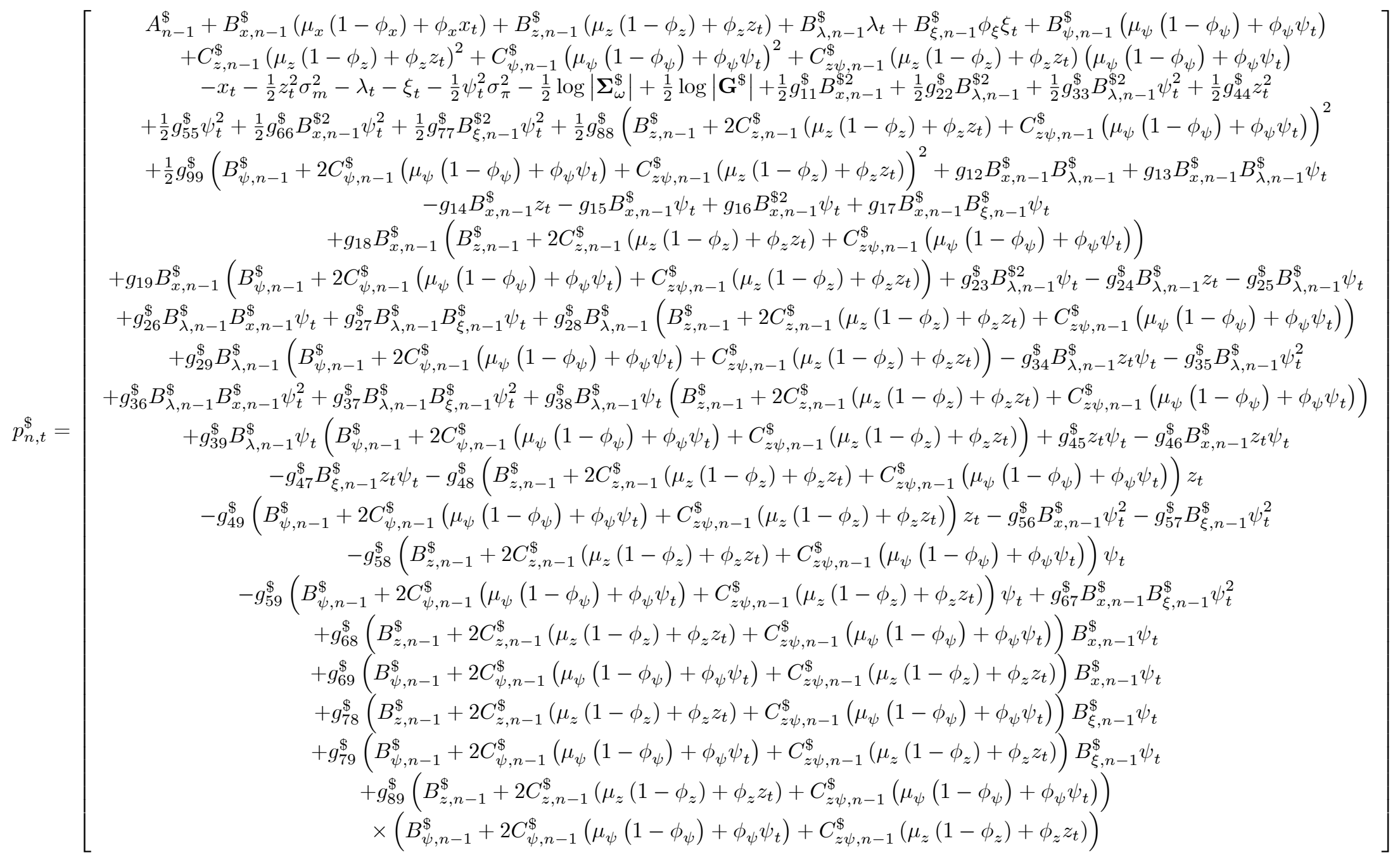

Thus, the coefficients of the pricing equation satisfy 


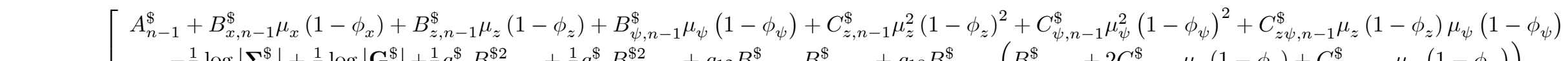

$$
\begin{aligned}
& -\frac{1}{2} \log \left|\mathbf{\Sigma}_{\omega}^{\$}\right|+\frac{1}{2} \log \left|\mathbf{G}^{\$}\right|+\frac{1}{2} g_{11}^{\$} B_{x, n-1}^{\$ 2}+\frac{1}{2} g_{22}^{\$} B_{\lambda, n-1}^{\$ 2}+g_{12} B_{x, n-1}^{\$} B_{\lambda, n-1}^{\$}+g_{18} B_{x, n-1}^{\$}\left(B_{z, n-1}^{\$}+2 C_{z, n-1}^{\$} \mu_{z}\left(1-\phi_{z}\right)+C_{z \psi, n-1}^{\$} \mu_{\psi}\left(1-\phi_{\psi}\right)\right) \\
& +g_{19} B_{x, n-1}^{\$}\left(B_{\psi, n-1}^{\$}+2 C_{\psi, n-1}^{\$} \mu_{\psi}\left(1-\phi_{\psi}\right)+C_{z \psi, n-1}^{\$} \mu_{z}\left(1-\phi_{z}\right)\right)+\frac{1}{2} g_{88}^{\$}\left(B_{z, n-1}^{\$}+2 C_{z, n-1}^{\$} \mu_{z}\left(1-\phi_{z}\right)+C_{z \psi, n-1}^{\$} \mu_{\psi}\left(1-\phi_{\psi}\right)\right)^{2} \\
& +\frac{1}{2} g_{99}^{\$}\left(B_{\psi, n-1}^{\$}+2 C_{\psi, n-1}^{\$} \mu_{\psi}\left(1-\phi_{\psi}\right)+C_{z \psi, n-1}^{\$} \mu_{z}\left(1-\phi_{z}\right)\right)^{2}+g_{28}^{\$}\left(B_{z, n-1}^{\$}+2 C_{z, n-1}^{\$} \mu_{z}\left(1-\phi_{z}\right)+C_{z \psi, n-1}^{\$} \mu_{\psi}\left(1-\phi_{\psi}\right)\right) B_{\lambda, n-1}^{\$} \\
& +g_{29}^{\$}\left(B_{\psi, n-1}^{\$}+2 C_{\psi, n-1}^{\$} \mu_{\psi}\left(1-\phi_{\psi}\right)+C_{z \psi, n-1}^{\$} \mu_{z}\left(1-\phi_{z}\right)\right) B_{\lambda, n-1}^{\$} \\
& +g_{89}^{\$}\left(B_{z, n-1}^{\$}+2 C_{z, n-1}^{\$} \mu_{z}\left(1-\phi_{z}\right)+C_{z \psi, n-1}^{\$} \mu_{\psi}\left(1-\phi_{\psi}\right)\right)\left(B_{\psi, n-1}^{\$}+2 C_{\psi, n-1}^{\$} \mu_{\psi}\left(1-\phi_{\psi}\right)+C_{z \psi, n-1}^{\$} \mu_{z}\left(1-\phi_{z}\right)\right) \\
& B_{x, n}^{\$}=B_{x, n-1}^{\$} \phi_{x}-1 \\
& B_{\lambda, n}^{\$}=B_{\lambda, n-1}^{\$}-1 \\
& B_{\xi, n}^{\$}=B_{\xi, n-1}^{\$} \phi_{\xi}-1
\end{aligned}
$$

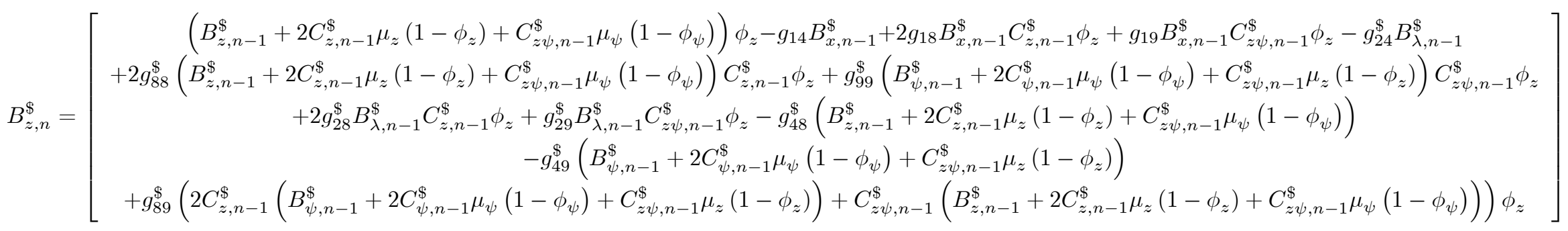

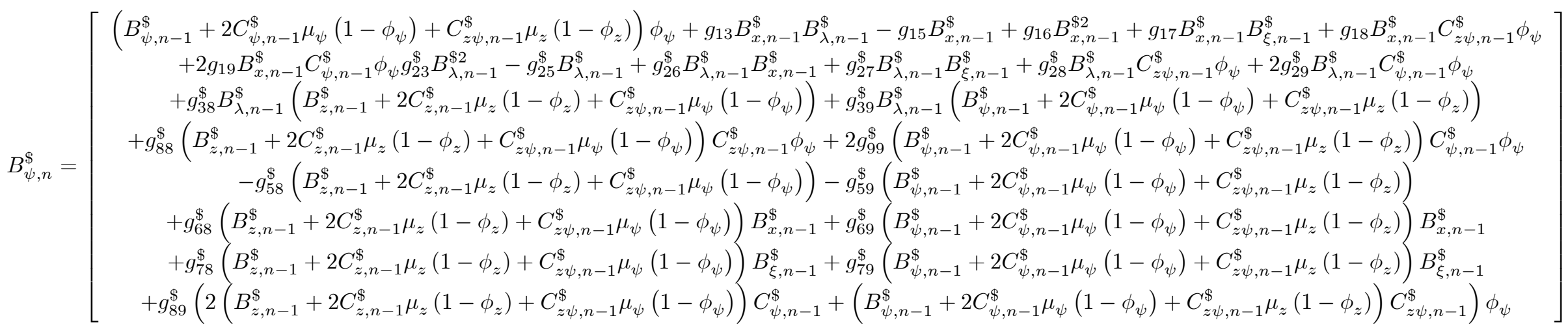




$$
\begin{aligned}
& C_{z, n}^{\$}=\left[C_{z, n-1}^{\$} \phi_{z}^{2}-\frac{1}{2} \sigma_{m}^{2}+\frac{1}{2} g_{44}^{\$}+2 g_{88}^{\$} C_{z, n-1}^{\$ 2} \phi_{z}^{2}+\frac{1}{2} g_{99}^{\$} C_{z \psi, n-1}^{\$ 2} \phi_{z}^{2}-2 g_{48}^{\$} C_{z, n-1}^{\$} \phi_{z}-g_{49}^{\$} C_{z \psi, n-1}^{\$} \phi_{z}+2 g_{89}^{\$} C_{z, n-1}^{\$} C_{z \psi, n-1}^{\$} \phi_{z}^{2}\right]
\end{aligned}
$$

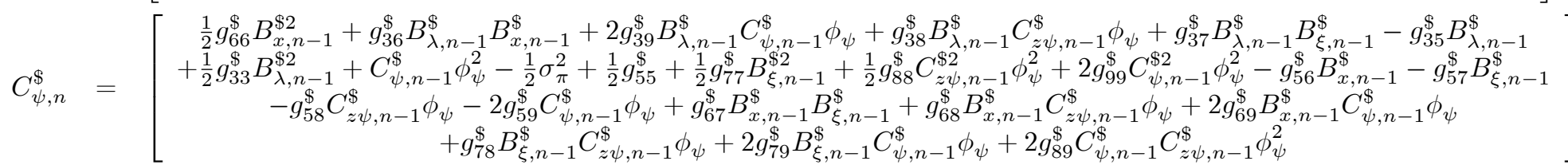

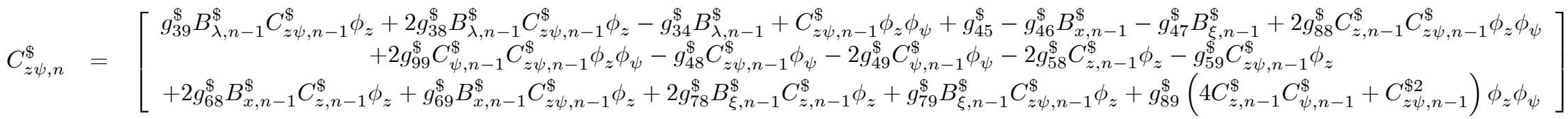

where $B_{x, 1}^{\$}=-1, B_{\lambda, 1}^{\$}=-1, B_{\xi, 1}^{\$}=-1, C_{z \psi, 1}^{\$}=\sigma_{m \pi}$ and all other coefficients are zero at $n=1$. 


\section{A.2.3 Expected Excess Returns}

Real Bond Premia The log expected gross excess return on an $n$-period zero-coupon real bond is $\log E_{t}\left[\frac{P_{n-1, t+1}}{P_{n, t}}\right]-E_{t}\left[r_{1, t+1}\right]=\log E_{t}\left[\exp \left\{p_{n-1 . t+1}-p_{n, t}\right\}\right]-x_{t}$

$$
\begin{gathered}
{\left[\begin{array}{r}
A_{n-1}-A_{n}+B_{x, n-1} \mu_{x}\left(1-\phi_{x}\right)+B_{z, n-1} \mu_{z}\left(1-\phi_{z}\right)+B_{\psi, n-1} \mu_{\psi}\left(1-\phi_{\psi}\right) \\
+C_{z, n-1} \mu_{z}^{2}\left(1-\phi_{z}\right)^{2}+C_{\psi, n-1} \mu_{\psi}^{2}\left(1-\phi_{\psi}\right)^{2}+C_{z \psi, n-1} \mu_{z}\left(1-\phi_{z}\right) \mu_{\psi}\left(1-\phi_{\psi}\right) \\
+\left(B_{x, n-1} \phi_{x}-B_{x, n}-1\right) x_{t}+\left(C_{z, n-1} \phi_{z}^{2}-C_{z, n}\right) z_{t}^{2}+\left(C_{\psi, n-1} \phi_{\psi}^{2}-C_{\psi, n}\right) \psi_{t}^{2}+\left(C_{z \psi, n-1} \phi_{z} \phi_{\psi}-C_{z \psi, n}\right) z_{t} \psi_{t} \\
+\left(B_{z, n-1} \phi_{z}-B_{z, n}+2 C_{z, n-1} \mu_{z}\left(1-\phi_{z}\right) \phi_{z}+C_{z \psi, n-1} \mu_{\psi}\left(1-\phi_{\psi}\right) \phi_{z}\right) z_{t} \\
+\left(B_{\psi, n-1} \phi_{\psi}-B_{\psi, n}+2 C_{\psi, n-1} \mu_{\psi}\left(1-\phi_{\psi}\right) \phi_{\psi}+C_{z \psi, n-1} \mu_{z}\left(1-\phi_{z}\right) \phi_{\psi}\right) \psi_{t}
\end{array}\right]} \\
+\log E_{t}\left[\exp \left\{\begin{array}{c}
B_{x, n-1} \psi_{t} \varepsilon_{x, t+1}+B_{x, n-1} \varepsilon_{X, t+1}+C_{z, n-1} \varepsilon_{z, t+1}^{2}+C_{\psi, n-1} \varepsilon_{\psi, t+1}^{2}+C_{z \psi, n-1} \varepsilon_{z, t+1} \varepsilon_{\psi, t+1} \\
+\left(B_{z, n-1}+2 C_{z, n-1}\left(\mu_{z}\left(1-\phi_{z}\right)+\phi_{z} z_{t}\right)+C_{z \psi, n-1}\left(\mu_{\psi}\left(1-\phi_{\psi}\right)+\phi_{\psi} \psi_{t}\right)\right) \varepsilon_{z, t+1} \\
+\left(B_{\psi, n-1}+2 C_{\psi, n-1}\left(\mu_{\psi}\left(1-\phi_{\psi}\right)+\phi_{\psi} \psi_{t}\right)+C_{z \psi, n-1}\left(\mu_{z}\left(1-\phi_{z}\right)+\phi_{z} z_{t}\right)\right) \varepsilon_{\psi, t+1}
\end{array}\right\}\right]
\end{gathered}
$$

since the shocks are conditionally jointly normal. Note that the coefficient recursion implies that $B_{x, n}=B_{x, n-1} \phi_{x}-1$ so that the terms involving $x_{t}$ drop out. Following Campbell, Chan, and Viceira (2003), we calculate the expectation by completing the square. Let $\boldsymbol{\nu}^{\prime}=\left(\varepsilon_{X, t+1}, \varepsilon_{x, t+1}, \varepsilon_{z, t+1}, \varepsilon_{\psi, t+1}\right) \sim N\left(0, \boldsymbol{\Sigma}_{v}\right)$,

$$
\begin{aligned}
& \mathbf{f}_{1}=\left(\begin{array}{c}
B_{x, n-1} \\
B_{x, n-1} \psi_{t} \\
\left(B_{z, n-1}+2 C_{z, n-1}\left(\mu_{z}\left(1-\phi_{z}\right)+\phi_{z} z_{t}\right)+C_{z \psi, n-1}\left(\mu_{\psi}\left(1-\phi_{\psi}\right)+\phi_{\psi} \psi_{t}\right)\right) \\
\left(B_{\psi, n-1}+2 C_{\psi, n-1}\left(\mu_{\psi}\left(1-\phi_{\psi}\right)+\phi_{\psi} \psi_{t}\right)+C_{z \psi, n-1}\left(\mu_{z}\left(1-\phi_{z}\right)+\phi_{z} z_{t}\right)\right)
\end{array}\right) \\
& \mathbf{F}_{2}=\left(\begin{array}{ccc}
0 & 0 & 0 \\
0 & C_{z, n-1} & \frac{1}{2} C_{z \psi, n-1} \\
0 & \frac{1}{2} C_{z \psi, n-1} & C_{\psi, n-1}
\end{array}\right)
\end{aligned}
$$

Then

$$
E_{t}\left[\exp \left\{\mathbf{f}_{1}^{\prime} \boldsymbol{\nu}+\boldsymbol{\nu}^{\prime} \mathbf{F}_{2} \boldsymbol{\nu}\right\}\right]=\exp \left\{-\frac{1}{2} \log \left|\boldsymbol{\Sigma}_{\nu}\right|+\frac{1}{2} \log |\mathbf{H}|+\frac{1}{2} \mathbf{f}_{1} \mathbf{H f}_{1}^{\prime}\right\}
$$

where $\mathbf{H}=\left(\boldsymbol{\Sigma}_{\nu}^{-1}-2 \mathbf{F}_{2}\right)^{-1}$ 
Let $h_{i j}$ be the $i j$-th element of $\mathbf{H}$. Then expanding and collecting terms gives

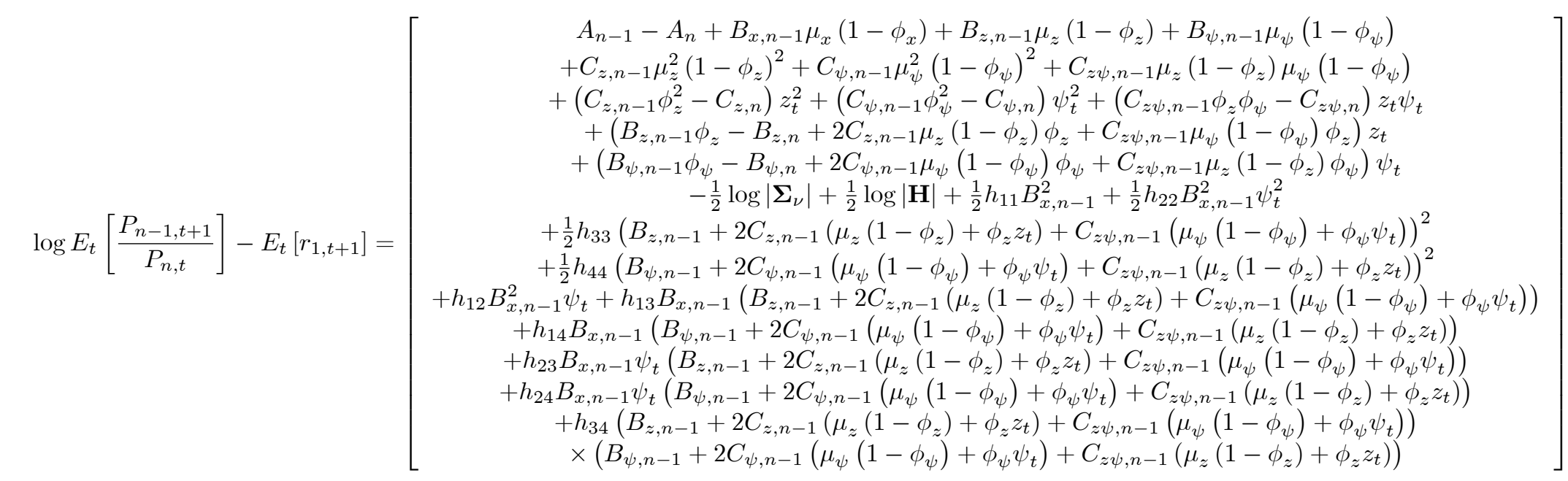

Thus, we can write

$$
\log E_{t}\left[\frac{P_{n-1, t+1}}{P_{n, t}}\right]-E_{t}\left[r_{1, t+1}\right]=\kappa_{n}+\eta_{z, n} z_{t}+\eta_{\psi, n} \psi_{t}+\beta_{z, n} z_{t}^{2}+\beta_{\psi, n} \psi_{t}^{2}+\beta_{z \psi, n} z_{t} \psi_{t}
$$

where the coefficients are given by

$$
\begin{aligned}
& \kappa_{n}=\left[\begin{array}{c}
A_{n-1}-A_{n}+B_{x, n-1} \mu_{x}\left(1-\phi_{x}\right)+B_{z, n-1} \mu_{z}\left(1-\phi_{z}\right)+B_{\psi, n-1} \mu_{\psi}\left(1-\phi_{\psi}\right)+C_{z, n-1} \mu_{z}^{2}\left(1-\phi_{z}\right)^{2}+C_{\psi, n-1} \mu_{\psi}^{2}\left(1-\phi_{\psi}\right)^{2} \\
+C_{z \psi, n-1} \mu_{z}\left(1-\phi_{z}\right) \mu_{\psi}\left(1-\phi_{\psi}\right)-\frac{1}{2} \log \left|\boldsymbol{\Sigma}_{\nu}\right|+\frac{1}{2} \log |\mathbf{H}|+\frac{1}{2} h_{11} B_{x, n-1}^{2}+\frac{1}{2} h_{33}\left(B_{z, n-1}+2 C_{z, n-1} \mu_{z}\left(1-\phi_{z}\right)+C_{z \psi, n-1} \mu_{\psi}\left(1-\phi_{\psi}\right)\right)^{2} \\
+\frac{1}{2} h_{44}\left(B_{\psi, n-1}+2 C_{\psi, n-1} \mu_{\psi}\left(1-\phi_{\psi}\right)+C_{z \psi, n-1} \mu_{z}\left(1-\phi_{z}\right)\right)^{2}+h_{13} B_{x, n-1}\left(B_{z, n-1}+2 C_{z, n-1} \mu_{z}\left(1-\phi_{z}\right)+C_{z \psi, n-1} \mu_{\psi}\left(1-\phi_{\psi}\right)\right) \\
+h_{14} B_{x, n-1}\left(B_{\psi, n-1}+2 C_{\psi, n-1} \mu_{\psi}\left(1-\phi_{\psi}\right)+C_{z \psi, n-1} \mu_{z}\left(1-\phi_{z}\right)\right) \\
+h_{34}\left(B_{z, n-1}+2 C_{z, n-1} \mu_{z}\left(1-\phi_{z}\right)+C_{z \psi, n-1} \mu_{\psi}\left(1-\phi_{\psi}\right)\right)\left(B_{\psi, n-1}+2 C_{\psi, n-1} \mu_{\psi}\left(1-\phi_{\psi}\right)+C_{z \psi, n-1} \mu_{z}\left(1-\phi_{z}\right)\right)
\end{array}\right] \\
& \eta_{z, n}=\left[\begin{array}{c}
B_{z, n-1} \phi_{z}-B_{z, n}+2 C_{z, n-1} \mu_{z}\left(1-\phi_{z}\right) \phi_{z}+C_{z \psi, n-1} \mu_{\psi}\left(1-\phi_{\psi}\right) \phi_{z}+2 h_{33}\left(B_{z, n-1}+2 C_{z, n-1} \mu_{z}\left(1-\phi_{z}\right)+C_{z \psi, n-1} \mu_{\psi}\left(1-\phi_{\psi}\right)\right) C_{z, n-1} \phi_{z} \\
+h_{44}\left(B_{\psi, n-1}+2 C_{\psi, n-1} \mu_{\psi}\left(1-\phi_{\psi}\right)+C_{z \psi, n-1} \mu_{z}\left(1-\phi_{z}\right)\right) C_{z \psi, n-1} \phi_{z}+2 h_{13} B_{x, n-1} C_{z, n-1} \phi_{z}+h_{14} B_{x, n-1} C_{z \psi, n-1} \phi_{z} \\
+h_{34}\left[2 C_{z, n-1}\left(B_{\psi, n-1}+2 C_{\psi, n-1} \mu_{\psi}\left(1-\phi_{\psi}\right)+C_{z \psi, n-1} \mu_{z}\left(1-\phi_{z}\right)\right)+C_{z \psi, n-1}\left(B_{z, n-1}+2 C_{z, n-1} \mu_{z}\left(1-\phi_{z}\right)+C_{z \psi, n-1} \mu_{\psi}\left(1-\phi_{\psi}\right)\right)\right] \phi_{z}
\end{array}\right] \\
& \eta_{\psi, n}=\left[\begin{array}{c}
B_{\psi, n-1} \phi_{\psi}-B_{\psi, n}+2 C_{\psi, n-1} \mu_{\psi}\left(1-\phi_{\psi}\right) \phi_{\psi}+C_{z \psi, n-1} \mu_{z}\left(1-\phi_{z}\right) \phi_{\psi}+h_{33}\left(B_{z, n-1}+2 C_{z, n-1} \mu_{z}\left(1-\phi_{z}\right)+C_{z \psi, n-1} \mu_{\psi}\left(1-\phi_{\psi}\right)\right) C_{z \psi, n-1} \phi_{\psi} \\
+2 h_{44}\left(B_{\psi, n-1}+2 C_{\psi, n-1} \mu_{\psi}\left(1-\phi_{\psi}\right)+C_{z \psi, n-1} \mu_{z}\left(1-\phi_{z}\right)\right) C_{\psi, n-1} \phi_{\psi}+h_{12} B_{x, n-1}^{2} \psi_{t}+h_{13} B_{x, n-1} C_{z \psi, n-1} \phi_{\psi} \psi_{t}+2 h_{14} B_{x, n-1} C_{\psi, n-1} \phi_{\psi} \psi_{t} \\
+h_{23} B_{x, n-1}\left(B_{z, n-1}+2 C_{z, n-1} \mu_{z}\left(1-\phi_{z}\right)+C_{z \psi, n-1} \mu_{\psi}\left(1-\phi_{\psi}\right)\right)+h_{24} B_{x, n-1}\left(B_{\psi, n-1}+2 C_{\psi, n-1} \mu_{\psi}\left(1-\phi_{\psi}\right)+C_{z \psi, n-1} \mu_{z}\left(1-\phi_{z}\right)\right) \\
+h_{34}\left[2 C_{\psi, n-1}\left(B_{z, n-1}+2 C_{z, n-1} \mu_{z}\left(1-\phi_{z}\right)+C_{z \psi, n-1} \mu_{\psi}\left(1-\phi_{\psi}\right)\right)+C_{z \psi, n-1}\left(B_{\psi, n-1}+2 C_{\psi, n-1} \mu_{\psi}\left(1-\phi_{\psi}\right)+C_{z \psi, n-1} \mu_{z}\left(1-\phi_{z}\right)\right)\right] \phi_{\psi}
\end{array}\right]
\end{aligned}
$$




$$
\begin{aligned}
& \beta_{z, n}=\left[\left(C_{z, n-1} \phi_{z}^{2}-C_{z, n}\right)+2 h_{33} C_{z, n-1}^{2} \phi_{z}^{2}+\frac{1}{2} h_{44} C_{z \psi, n-1}^{2} \phi_{z}^{2}+2 h_{34} C_{z, n-1} C_{z \psi, n-1} \phi_{z}^{2}\right] \\
& \beta_{\psi, n}=\left[\left(C_{\psi, n-1} \phi_{\psi}^{2}-C_{\psi, n}\right)+\frac{1}{2} h_{33} C_{z \psi, n-1}^{2} \phi_{\psi}^{2}+2 h_{44} C_{\psi, n-1}^{2} \phi_{\psi}^{2}+h_{23} B_{x, n-1} C_{z \psi, n-1} \phi_{\psi}+2 h_{24} B_{x, n-1} C_{\psi, n-1} \phi_{\psi}+h_{34} 2 C_{\psi, n-1} C_{z \psi, n-1} \phi_{\psi}^{2}\right] \\
& \beta_{z \psi, n}=\left[\begin{array}{c}
\left(C_{z \psi, n-1} \phi_{z} \phi_{\psi}-C_{z \psi, n}\right)+2 h_{33} C_{z, n-1} C_{z \psi, n-1} \phi_{z} \phi_{\psi}+2 h_{44} C_{\psi, n-1 t} C_{z \psi, n-1} \phi_{z} \phi_{\psi}+2 h_{23} B_{x, n-1} C_{z, n-1} \phi_{z} \\
+h_{24} B_{x, n-1} C_{z \psi, n-1} \phi_{z}+h_{34} C_{z \psi, n-1}^{2} \phi_{\psi} \phi_{z}
\end{array}\right]
\end{aligned}
$$

Nominal Bond Premia The log conditional expected real return on a 1-period zero-coupon nominal bond is

$$
E_{t}\left[r_{1, t+1}^{\$}-\pi_{t+1}\right]=-\sigma_{m, \pi} z_{t} \psi_{t}
$$

The log conditional expected gross excess return on an $n$-period zero-coupon nominal bond is

$$
\begin{aligned}
& \log E_{t}\left[\frac{P_{n-1, t+1}^{\$}}{P_{n, t}^{\$}}\right]-E_{t}\left[r_{1, t+1}^{\$}\right]=\log E_{t}\left[\exp \left\{p_{n-1 . t+1}^{\$}-p_{n, t}^{\$}\right\}\right]-x_{t}-\lambda_{t}-\xi_{t}+\sigma_{m, \pi} z_{t} \psi_{t}
\end{aligned}
$$

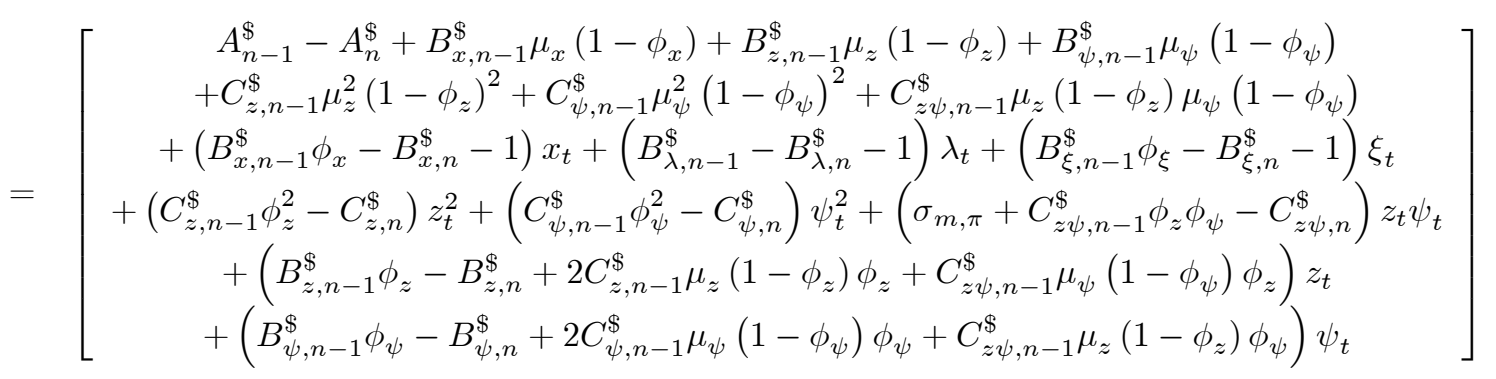

$$
\begin{aligned}
& +\log E_{t}\left[\exp \left\{\begin{array}{c}
B_{x, n-1}^{\$} \psi_{t} \varepsilon_{x, t+1}+B_{x, n-1}^{\$} \varepsilon_{X, t+1}+B_{\lambda, n-1}^{\$} \psi_{t} \varepsilon_{\lambda, t+1}+B_{\lambda, n-1}^{\$} \varepsilon_{\Lambda, t+1}+B_{\xi, n-1}^{\$} \psi_{t} \varepsilon_{\xi, t+1} \\
+C_{z, n-1}^{\$} \varepsilon_{z, t+1}^{2}+C_{\psi, n-1}^{\$} \varepsilon_{\psi, t+1}^{2}+C_{z \psi, n-1}^{\$} \varepsilon_{z, t+1} \varepsilon_{\psi, t+1} \\
+\left(B_{z, n-1}^{\$}+2 C_{z, n-1}^{\$}\left(\mu_{z}\left(1-\phi_{z}\right)+\phi_{z} z_{t}\right)+C_{z \psi, n-1}^{\$}\left(\mu_{\psi}\left(1-\phi_{\psi}\right)+\phi_{\psi} \psi_{t}\right)\right) \varepsilon_{z, t+1} \\
+\left(B_{\psi, n-1}^{\$}+2 C_{\psi, n-1}^{\$}\left(\mu_{\psi}\left(1-\phi_{\psi}\right)+\phi_{\psi} \psi_{t}\right)+C_{z \psi, n-1}^{\$}\left(\mu_{z}\left(1-\phi_{z}\right)+\phi_{z} z_{t}\right)\right) \varepsilon_{\psi, t+1}
\end{array}\right\}\right]
\end{aligned}
$$

Note that the coefficient recursions imply that $B_{x, n}^{\$}=B_{x, n-1}^{\$} \phi_{x}-1, B_{\lambda, n}^{\$}=B_{\lambda, n-1}^{\$}-1$, and $B_{\xi, n}^{\$}=B_{\xi, n-1}^{\$} \phi_{\xi}-1$, so that the terms involving $x_{t}, \lambda_{t}$, and $\xi_{t}$ drop out. Following Campbell, Chan, and Viceira (2003), we calculate the expectation by completing the square. Let 
$\boldsymbol{\nu}^{\$ \prime}=\left(\varepsilon_{X, t+1}, \varepsilon_{\Lambda, t+1}, \varepsilon_{x, t+1}, \varepsilon_{\lambda, t+1}, \varepsilon_{\xi, t+1}, \varepsilon_{z, t+1}, \varepsilon_{\psi, t+1}\right)^{\sim} N\left(0, \boldsymbol{\Sigma}_{v}^{\$}\right)$

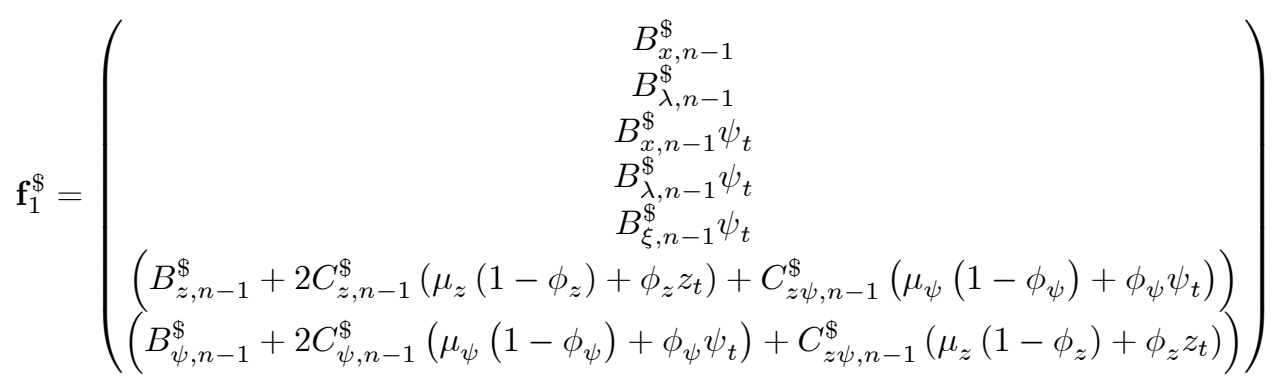

$$
\begin{aligned}
& \mathbf{F}_{2}^{\$}=\left(\begin{array}{ccccc}
0 & & \cdots & & 0 \\
& \ddots & & & \\
\vdots & & & & \\
& & & C_{z, n-1}^{\$} & \frac{1}{2} C_{z \psi, n-1}^{\$} \\
0 & & & \frac{1}{2} C_{z \psi, n-1}^{\Phi} & C_{\psi, n-1}^{\$}
\end{array}\right)
\end{aligned}
$$

Then

$$
E_{t}\left[\exp \left\{\mathbf{f}_{1}^{\$ \prime} \boldsymbol{\nu}^{\$}+\boldsymbol{\nu}^{\$ \prime} \mathbf{F}_{2}^{\$} \boldsymbol{\nu}^{\$}\right\}\right]=\exp \left\{-\frac{1}{2} \log \left|\boldsymbol{\Sigma}_{\nu}^{\$}\right|+\frac{1}{2} \log \left|\mathbf{H}^{\$}\right|+\frac{1}{2} \mathbf{f}_{1}^{\$} \mathbf{H}^{\$} \mathbf{f}_{1}^{\$ \prime}\right\}
$$

where $\mathbf{H}^{\$}=\left(\boldsymbol{\Sigma}_{\nu}^{\$-1}-2 \mathbf{F}_{2}^{\$}\right)^{-1}$.

Let $h_{i j}^{\$}$ be the $i j$-th element of $\mathbf{H}^{\$}$. Then expanding and collecting terms gives 


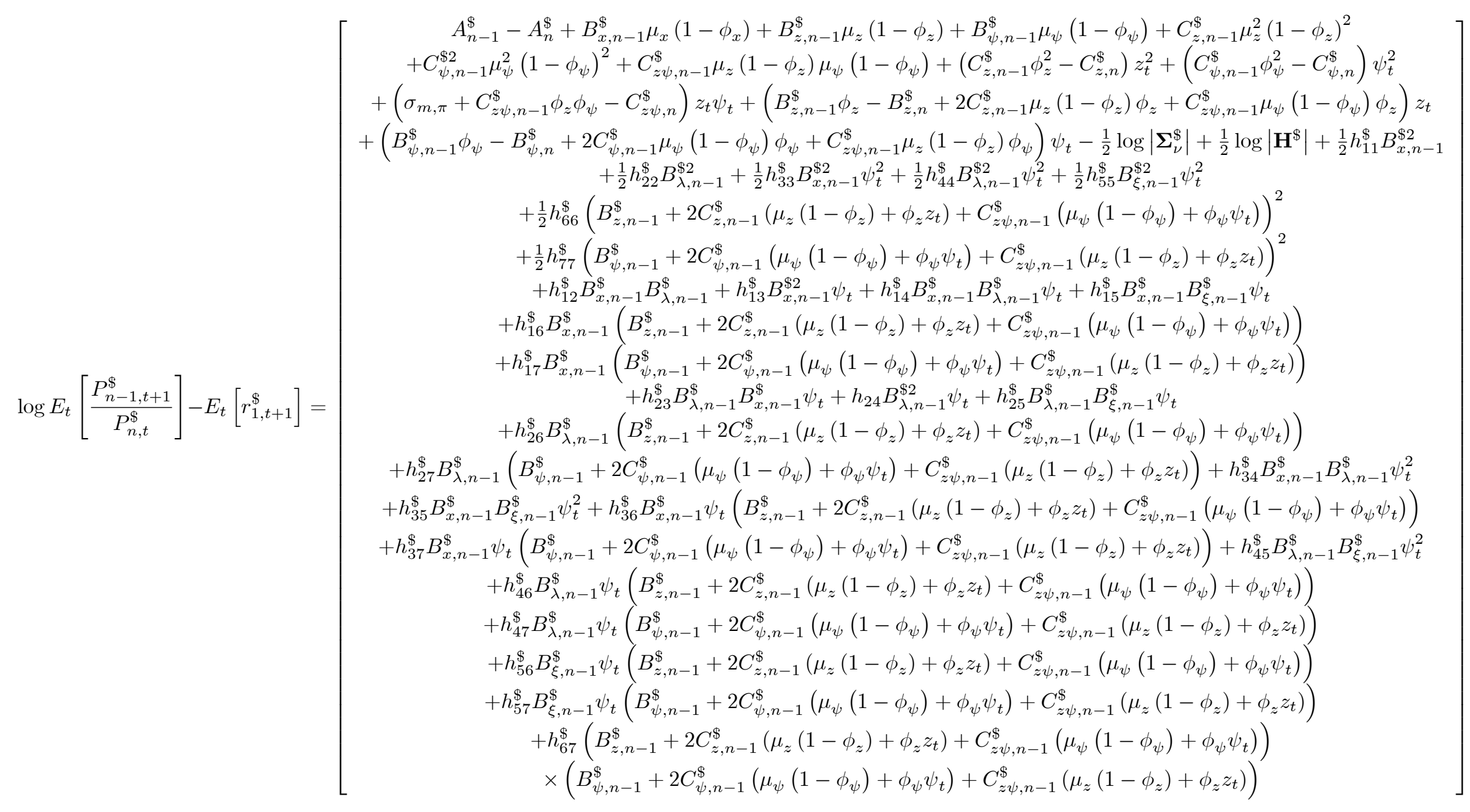

Thus, we can write

$$
\log E_{t}\left[\frac{P_{n-1, t+1}^{\S}}{P_{n, t}^{\$}}\right]-E_{t}\left[r_{1, t+1}^{\$}\right]=\kappa_{n}^{\$}+\eta_{z, n}^{\$} z_{t}+\eta_{\psi, n}^{\$} \psi_{t}+\beta_{z, n}^{\S} z_{t}^{2}+\beta_{\psi, n}^{\$} \psi_{t}^{2}+\beta_{z \psi, n}^{\S} z_{t} \psi_{t}
$$

where the coefficients are given by 


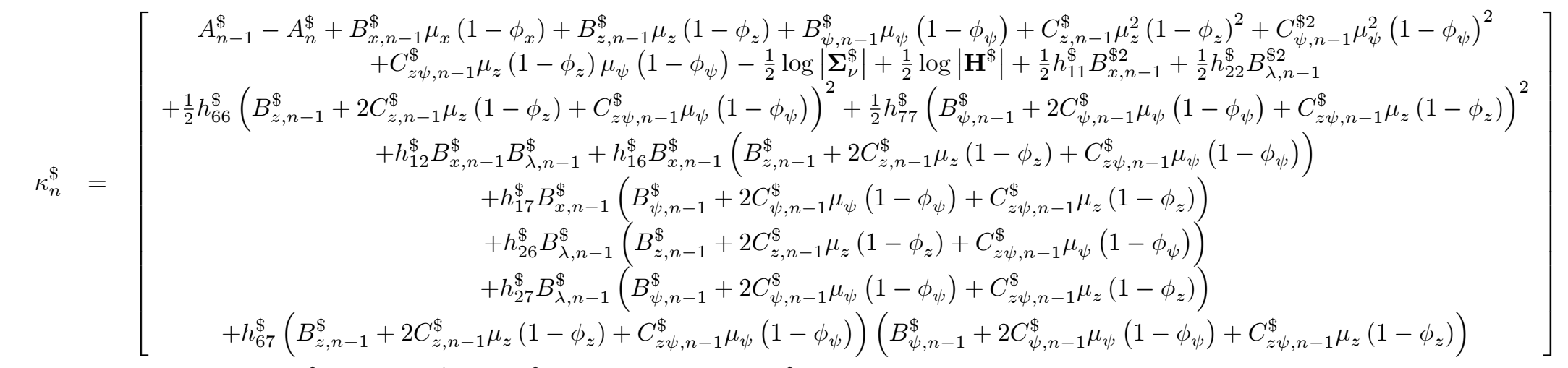

$$
\begin{aligned}
& B_{z, n-1}^{\$} \phi_{z}-B_{z, n}^{\$}+2 C_{z, n-1}^{\$} \mu_{z}\left(1-\phi_{z}\right) \phi_{z}+C_{z \psi, n-1}^{\$} \mu_{\psi}\left(1-\phi_{\psi}\right) \phi_{z} \\
& +2 h_{66}^{\$}\left(B_{z, n-1}^{\$}+2 C_{z, n-1}^{\$} \mu_{z}\left(1-\phi_{z}\right)+C_{z \psi, n-1}^{\$} \mu_{\psi}\left(1-\phi_{\psi}\right)\right) C_{z, n-1}^{\$} \phi_{z} \\
& \eta_{z, n}^{\$}=\quad \begin{array}{c}
+h_{77}^{\$}\left(B_{\psi, n-1}^{\$}+2 C_{\psi, n-1}^{\$} \mu_{\psi}\left(1-\phi_{\psi}\right)+C_{z \psi, n-1}^{\$} \mu_{z}\left(1-\phi_{z}\right)\right) C_{z \psi, n-1}^{\$} \phi_{z} \\
+2 h_{16}^{\$} B_{x, n-1}^{\Phi} C_{z, n-1}^{\$} \phi_{z}+h_{17}^{\$} B_{x, n-1}^{\$} C_{z \psi, n-1}^{\$} \phi_{z} 2 h_{26}^{\$} B_{\lambda, n-1}^{\$} C_{z, n-1}^{\$} \phi_{z}+h_{27}^{\$} B_{\lambda, n-1}^{\$} C_{z \psi, n-1}^{\$} \phi_{z}
\end{array} \\
& +h_{67}^{\$}\left(\begin{array}{c}
\left(B_{z, n-1}^{\$}+2 C_{z, n-1}^{\$} \mu_{z}\left(1-\phi_{z}\right)+C_{z \psi, n-1}^{\Phi} \mu_{\psi}\left(1-\phi_{\psi}\right)\right) C_{z \psi, n-1}^{\$} \phi_{z} \\
+2 C_{z, n-1}^{\$} \phi_{z}\left(B_{\psi, n-1}^{\$}+2 C_{\psi, n-1}^{\$} \mu_{\psi}\left(1-\phi_{\psi}\right)+C_{z \psi, n-1}^{\$} \mu_{z}\left(1-\phi_{z}\right)\right)
\end{array}\right)
\end{aligned}
$$

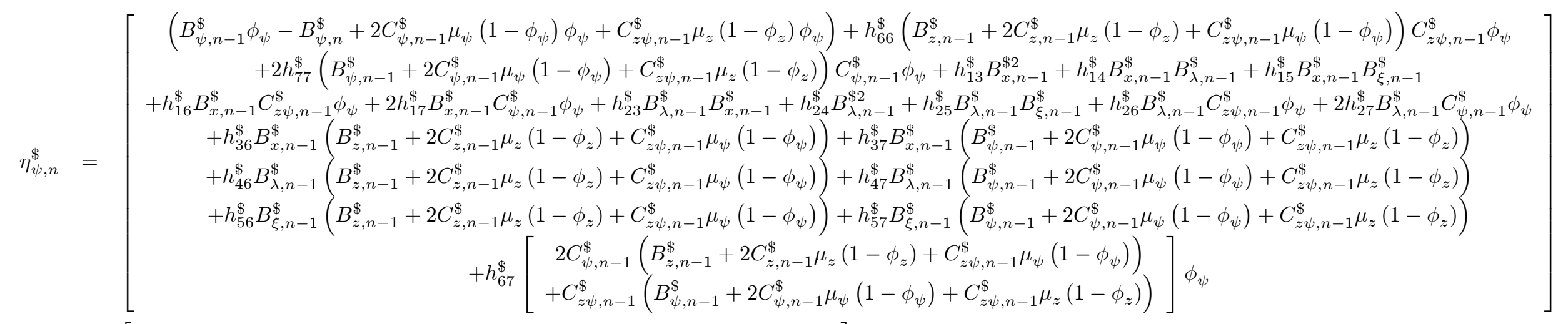

$$
\begin{aligned}
& \beta_{z, n}^{\$}=\left[C_{z, n-1}^{\$} \phi_{z}^{2}-C_{z, n}^{\$}+2 h_{66}^{\$} C_{z, n-1}^{\$ 2} \phi_{z}^{2}+\frac{1}{2} h_{77}^{\$} C_{z \psi, n-1}^{\$ 2} \phi_{z}^{2}+2 h_{67}^{\$} C_{z, n-1}^{\$} C_{z \psi, n-1}^{\$} \phi_{z}^{2}\right]
\end{aligned}
$$




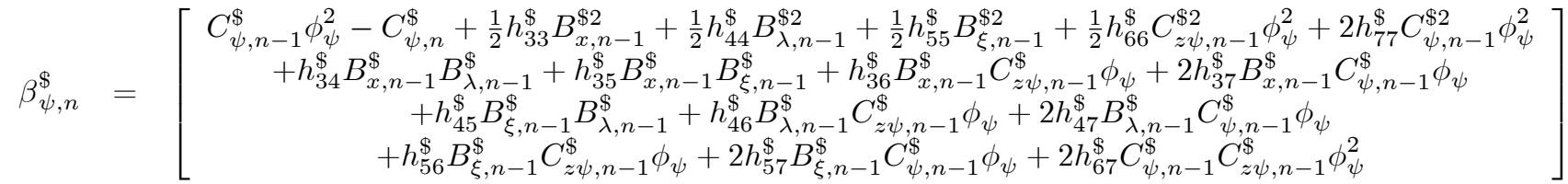

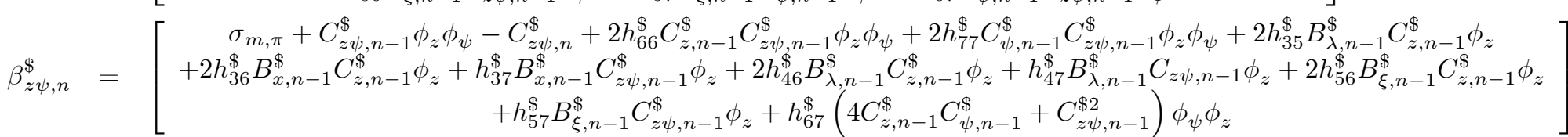




\section{A.2.4 Observation Equations}

Stock Returns We model the unexpected stock return as

$$
r_{e, t+1}-E_{t} r_{e, t+1}=\beta_{e x} \varepsilon_{x, t+1}+\beta_{e X} \varepsilon_{X, t+1}+\beta_{e m} \varepsilon_{m, t+1}
$$

We impose that the only non-zero covariance of $\varepsilon_{X, t+1}$ is $\sigma_{X, m}$. The standard pricing equation then implies that the expected equity return satisfies

$$
\begin{aligned}
1 & =E_{t}\left[\exp \left(r_{e, t+1}+m_{t+1}\right)\right] \\
& =\exp \left(E_{t} r_{e, t+1}-x_{t}-\frac{1}{2} z_{t}^{2} \sigma_{m}^{2}\right) \exp \left(\begin{array}{c}
\frac{1}{2} \beta_{e x}^{2} \sigma_{x}^{2}+\frac{1}{2} \beta_{e X}^{2} \sigma_{X}^{2}+\frac{1}{2} \beta_{e m}^{2} \sigma_{m}^{2}+\frac{1}{2} z_{t}^{2} \sigma_{m}^{2} \\
+\beta_{e x} \beta_{e m} \sigma_{x m}-\beta_{e x} z_{t} \sigma_{x m}+\beta_{e X} \beta_{e m} \sigma_{X, m}-\beta_{e X} z_{t} \sigma_{X m}-\beta_{e m} z_{t} \sigma_{m}^{2}
\end{array}\right)
\end{aligned}
$$

so that

$r_{e, t+1}=-\frac{1}{2} \beta_{e x}^{2} \sigma_{x}^{2}-\frac{1}{2} \beta_{e X}^{2} \sigma_{X}^{2}-\frac{1}{2} \beta_{e m}^{2} \sigma_{m}^{2}-\beta_{e x} \beta_{e m} \sigma_{x m}-\beta_{e X} \beta_{e m} \sigma_{X, m}+x_{t}+\left(\beta_{e x} \sigma_{x m}+\beta_{e X} \sigma_{X m}+\beta_{e m} \sigma_{m}^{2}\right) z_{t}+\beta_{e x} \varepsilon_{x, t+1}+\beta_{e X} \varepsilon_{X, t+1}+\beta_{e m} \varepsilon_{m, t+1}$

and

$$
E_{t}\left[r_{e, t+1}-r_{1, t+1}\right]-\frac{1}{2} \operatorname{Var}_{t}\left[r_{e, t+1}-r_{1, t+1}\right]=\left(\beta_{e x} \sigma_{x m}+\beta_{e X} \sigma_{X m}+\beta_{e m} \sigma_{m}^{2}\right) z_{t}
$$

Stock-Real Bond Return Covariance As we saw above, the holding period return on an $n$-period real bond is

$$
\begin{aligned}
r_{n, t+1}= & p_{n-1, t+1}-p_{n, t} \\
= & {\left[\begin{array}{c}
A_{n-1}-A_{n}+B_{x, n-1} \mu_{x}\left(1-\phi_{x}\right)+B_{z, n-1} \mu_{z}\left(1-\phi_{z}\right)+B_{\psi, n-1} \mu_{\psi}\left(1-\phi_{\psi}\right)+C_{z, n-1} \mu_{z}^{2}\left(1-\phi_{z}\right)^{2}+C_{\psi, n-1} \mu_{\psi}^{2}\left(1-\phi_{\psi}\right)^{2} \\
+C_{z \psi, n-1} \mu_{z}\left(1-\phi_{z}\right) \mu_{\psi}\left(1-\phi_{\psi}\right)+\left(B_{x, n-1} \phi_{x}-B_{x, n}-1\right) x_{t}+\left(C_{z, n-1} \phi_{z}^{2}-C_{z, n}\right) z_{t}^{2}+\left(C_{\psi, n-1} \phi_{\psi}^{2}-C_{\psi, n}\right) \psi_{t}^{2} \\
+\left(C_{z \psi, n-1} \phi_{z} \phi_{\psi}-C_{z \psi, n}\right) z_{t} \psi_{t}+\left(B_{z, n-1} \phi_{z}-B_{z, n}+2 C_{z, n-1} \mu_{z}\left(1-\phi_{z}\right) \phi_{z}+C_{z \psi, n-1} \mu_{\psi}\left(1-\phi_{\psi}\right) \phi_{z}\right) z_{t} \\
+\left(B_{\psi, n-1} \phi_{\psi}-B_{\psi, n}+2 C_{\psi, n-1} \mu_{\psi}\left(1-\phi_{\psi}\right) \phi_{\psi}+C_{z \psi, n-1} \mu_{z}\left(1-\phi_{z}\right) \phi_{\psi}\right) \psi_{t}
\end{array}\right] } \\
& +\left[\begin{array}{c}
B_{x, n-1} \psi_{t} \varepsilon_{x, t+1}+B_{x, n-1} \varepsilon_{X, t+1}+C_{z, n-1} \varepsilon_{z, t+1}^{2}+C_{\psi, n-1} \varepsilon_{\psi, t+1}^{2}+C_{z \psi, n-1} \varepsilon_{z, t+1} \varepsilon_{\psi, t+1} \\
+\left(B_{z, n-1}+2 C_{z, n-1}\left(\mu_{z}\left(1-\phi_{z}\right)+\phi_{z} z_{t}\right)+C_{z \psi, n-1}\left(\mu_{\psi}\left(1-\phi_{\psi}\right)+\phi_{\psi} \psi_{t}\right)\right) \varepsilon_{z, t+1} \\
+\left(B_{\psi, n-1}+2 C_{\psi, n-1}\left(\mu_{\psi}\left(1-\phi_{\psi}\right)+\phi_{\psi} \psi_{t}\right)+C_{z \psi, n-1}\left(\mu_{z}\left(1-\phi_{z}\right)+\phi_{z} z_{t}\right)\right) \varepsilon_{\psi, t+1}
\end{array}\right]
\end{aligned}
$$

We assume that the unexpected stock return is assumed to be

$$
r_{e, t+1}-E_{t} r_{e, t+1}=\beta_{e x} \varepsilon_{x, t+1}+\beta_{e X} \varepsilon_{X, t+1}+\beta_{e m} \varepsilon_{m, t+1}
$$

Since the $\varepsilon$ 's are conditionally jointly normal and mean zero we have $\operatorname{Cov}_{t}\left(\varepsilon_{a, t+1}, \varepsilon_{b, t+1}^{2}\right)=0$ and $\operatorname{Cov}_{t}\left(\varepsilon_{a, t+1}, \varepsilon_{b, t+1} \varepsilon_{c, t+1}\right)=0$ for all $a, b, c$. Furthermore, we impose that the only non-zero covariance of $\varepsilon_{X, t+1}$ is $\sigma_{X, m}$. Thus, the expression for the conditional covariance of stock returns with 
returns on a long-term real bond is

$$
\begin{aligned}
\operatorname{Cov}_{t}\left(r_{e, t+1}, r_{n, t+1}\right)= & \beta_{e x}\left(\begin{array}{c}
\left(B_{z, n-1}+2 C_{z, n-1} \mu_{z}\left(1-\phi_{z}\right)+C_{z \psi, n-1} \mu_{\psi}\left(1-\phi_{\psi}\right)\right) \sigma_{x . z} \\
+\left(B_{\psi, n-1}+2 C_{\psi, n-1} \mu_{\psi}\left(1-\phi_{\psi}\right)+C_{z \psi, n-1} \mu_{z}\left(1-\phi_{z}\right)\right) \sigma_{x, \psi}
\end{array}\right) \\
& +\beta_{e X} B_{x, n-1} \sigma_{X}^{2} \\
& +\beta_{e m}\left(\begin{array}{c}
B_{x, n-1} \sigma_{X m}+\left(B_{z, n-1}+2 C_{z, n-1} \mu_{z}\left(1-\phi_{z}\right)+C_{z \psi, n-1} \mu_{\psi}\left(1-\phi_{\psi}\right)\right) \sigma_{z, m} \\
+\left(B_{\psi, n-1}+2 C_{\psi, n-1} \mu_{\psi}\left(1-\phi_{\psi}\right)+C_{z \psi, n-1} \mu_{z}\left(1-\phi_{z}\right)\right) \sigma_{\psi, m}
\end{array}\right) \\
& +\left[\beta_{e x}\left(2 C_{z, n-1} \sigma_{x z} \phi_{z}+C_{z \psi, n-1} \sigma_{x \psi} \phi_{z}\right)+\beta_{e m}\left(2 C_{z, n-1} \sigma_{z m} \phi_{z}+C_{z \psi, n-1} \sigma_{\psi m} \phi_{z}\right)\right] z_{t} \\
& +\left[\beta_{e x}\left(B_{x, n-1} \sigma_{x}^{2}+C_{z \psi, n-1} \sigma_{x z} \phi_{\psi}+2 C_{\psi, n-1} \sigma_{x \psi} \phi_{\psi}\right)+\beta_{e m}\left(B_{x, n-1} \sigma_{x m}+C_{z \psi, n-1} \sigma_{z m} \phi_{\psi}+2 C_{\psi, n-1} \sigma_{\psi m} \phi_{\psi}\right)\right] \psi_{t}
\end{aligned}
$$

Stock-Nominal Bond Return Covariance As we saw above, the holding period return on an $n$-period nominal bond is

$$
\begin{aligned}
& r_{n, t+1}^{\$}=p_{n-1, t+1}^{\$}-p_{n, t}^{\$}
\end{aligned}
$$

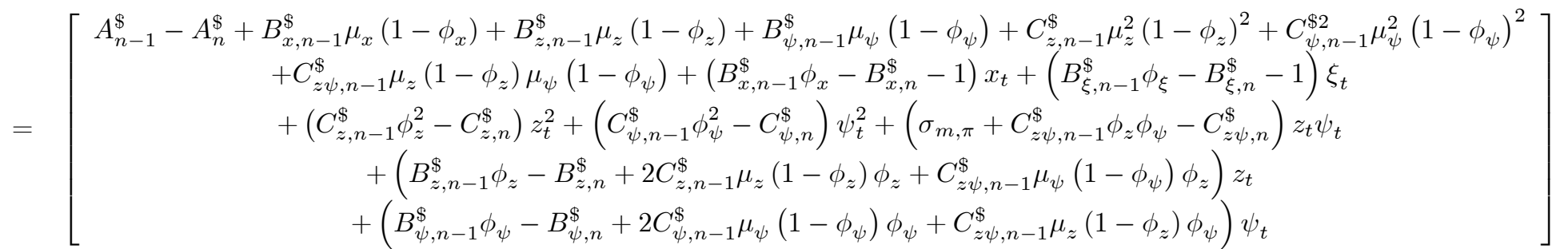

$$
\begin{aligned}
& +\left[\begin{array}{c}
B_{x, n-1}^{\$} \psi_{t} \varepsilon_{x, t+1}+B_{x, n-1}^{\$} \varepsilon_{X, t+1}+B_{\lambda, n-1}^{\$} \psi_{t} \varepsilon_{\lambda, t+1}+B_{\lambda, n-1}^{\$} \varepsilon_{\Lambda, t+1}+B_{\xi, n-1}^{\$} \psi_{t} \varepsilon_{\xi, t+1} \\
+C_{z, n-1}^{\Phi} \varepsilon_{z, t+1}^{2}+C_{\psi, n-1}^{\Phi} \varepsilon_{\psi, t+1}^{2}+C_{z \psi, n-1}^{\$} \varepsilon_{z, t+1} \varepsilon_{\psi, t+1} \\
+\left(B_{z, n-1}^{\$}+2 C_{z, n-1}^{\Phi}\left(\mu_{z}\left(1-\phi_{z}\right)+\phi_{z} z_{t}\right)+C_{z \psi, n-1}^{\Phi}\left(\mu_{\psi}\left(1-\phi_{\psi}\right)+\phi_{\psi} \psi_{t}\right)\right) \varepsilon_{z, t+1} \\
+\left(B_{\psi, n-1}^{\Phi}+2 C_{\psi, n-1}^{\Phi}\left(\mu_{\psi}\left(1-\phi_{\psi}\right)+\phi_{\psi} \psi_{t}\right)+C_{z \psi, n-1}^{\$}\left(\mu_{z}\left(1-\phi_{z}\right)+\phi_{z} z_{t}\right)\right) \varepsilon_{\psi, t+1}
\end{array}\right]
\end{aligned}
$$

We assume that the unexpected stock return is assumed to be

$$
r_{e, t+1}-E_{t} r_{e, t+1}=\beta_{e x} \varepsilon_{x, t+1}+\beta_{e X} \varepsilon_{X, t+1}+\beta_{e m} \varepsilon_{m, t+1}
$$

Thus, the conditional covariance with the real return on short term nominal bond is

$$
\operatorname{Cov}_{t}\left(r_{e, t+1}, r_{1, t+1}^{\$}-\pi_{t+1}\right)=\operatorname{Cov}\left(\beta_{e x} \varepsilon_{x, t+1}+\beta_{e X} \varepsilon_{X, t+1}+\beta_{e m} \varepsilon_{m, t+1},-\psi_{t} \varepsilon_{\pi, t+1}\right)=-\psi_{t}\left(\beta_{e x} \sigma_{x \pi}+\beta_{e m} \sigma_{m \pi}\right)
$$

since we impose the condition that the only non-zero covariance of $\varepsilon_{X, t+1}$ is $\sigma_{X, m}$. 
Again, the $\varepsilon$ 's are conditionally jointly normal and mean zero we have $\operatorname{Cov}_{t}\left(\varepsilon_{a, t+1}, \varepsilon_{b, t+1}^{2}\right)=0$ and $\operatorname{Cov}_{t}\left(\varepsilon_{a, t+1}, \varepsilon_{b, t+1} \varepsilon_{c, t+1}\right)=0$ for all $a, b, c$. Additionally, note that we impose $\sigma_{x, \Lambda}=0$ and that the only non-zero covariance of $\varepsilon_{\Lambda, t+1}$ is $\sigma_{\Lambda, m}$. Thus, the conditional covariance of stock returns with the returns on a long term nominal bond is

$$
\begin{aligned}
& \operatorname{Cov}_{t}\left(r_{e, t+1}, r_{n, t+1}^{\$}\right)=\beta_{e x}\left(\begin{array}{c}
\left(B_{z, n-1}^{\$}+2 C_{z, n-1}^{\$} \mu_{z}\left(1-\phi_{z}\right)+C_{z \psi, n-1}^{\$} \mu_{\psi}\left(1-\phi_{\psi}\right)\right) \sigma_{x . z} \\
+\left(B_{\psi, n-1}^{\$}+2 C_{\psi, n-1}^{\$} \mu_{\psi}\left(1-\phi_{\psi}\right)+C_{z \psi, n-1}^{\$} \mu_{z}\left(1-\phi_{z}\right)\right) \sigma_{x, \psi}
\end{array}\right) \\
& +\beta_{e X} B_{x, n-1}^{\$} \sigma_{X}^{2} \\
& +\beta_{e m}\left(\begin{array}{c}
B_{x, n-1}^{\$} \sigma_{X m}+B_{\lambda, n-1}^{\$} \sigma_{\Lambda m}+\left(B_{z, n-1}^{\$}+2 C_{z, n-1}^{\$} \mu_{z}\left(1-\phi_{z}\right)+C_{z \psi, n-1}^{\$} \mu_{\psi}\left(1-\phi_{\psi}\right)\right) \sigma_{z, m} \\
+\left(B_{\psi, n-1}^{\$}+2 C_{\psi, n-1}^{\$} \mu_{\psi}\left(1-\phi_{\psi}\right)+C_{z \psi, n-1}^{\$} \mu_{z}\left(1-\phi_{z}\right)\right) \sigma_{\psi, m}
\end{array}\right) \\
& +\left[\beta_{e x}\left(2 C_{z, n-1}^{\$} \sigma_{x z} \phi_{z}+C_{z \psi, n-1}^{\$} \sigma_{x \psi} \phi_{z}\right)+\beta_{e m}\left(2 C_{z, n-1}^{\$} \sigma_{z m} \phi_{z}+C_{z \psi, n-1}^{\$} \sigma_{\psi m} \phi_{z}\right)\right] z_{t} \\
& +\left[\begin{array}{c}
\beta_{e x}\left(B_{x, n-1}^{\$} \sigma_{x}^{2}+B_{\lambda, n-1}^{\$} \sigma_{x, \lambda}+B_{\xi, n-1}^{\$} \sigma_{x, \xi}+C_{z \psi, n-1}^{\$} \sigma_{x z} \phi_{\psi}+2 C_{\psi, n-1}^{\$} \sigma_{x \psi} \phi_{\psi}\right) \\
+\beta_{e m}\left(B_{x, n-1}^{\$} \sigma_{x m}+B_{\lambda, n-1}^{\$} \sigma_{m, \lambda}+B_{\xi, n-1}^{\$} \sigma_{m, \xi}+C_{z \psi, n-1}^{\$} \sigma_{z m} \phi_{\psi}+2 C_{\psi, n-1}^{\$} \sigma_{\psi m} \phi_{\psi}\right)
\end{array}\right] \psi_{t}
\end{aligned}
$$


Volatility of Real Bond Returns We have

$$
r_{n, t+1}-E_{t} r_{n, t+1}=\left[\begin{array}{c}
B_{x, n-1} \psi_{t} \varepsilon_{x, t+1}+B_{x, n-1} \varepsilon_{X, t+1}+C_{z, n-1} \varepsilon_{z, t+1}^{2}+C_{\psi, n-1} \varepsilon_{\psi, t+1}^{2}+C_{z \psi, n-1} \varepsilon_{z, t+1} \varepsilon_{\psi, t+1} \\
+\left(B_{z, n-1}+2 C_{z, n-1}\left(\mu_{z}\left(1-\phi_{z}\right)+\phi_{z} z_{t}\right)+C_{z \psi, n-1}\left(\mu_{\psi}\left(1-\phi_{\psi}\right)+\phi_{\psi} \psi_{t}\right)\right) \varepsilon_{z, t+1} \\
+\left(B_{\psi, n-1}+2 C_{\psi, n-1}\left(\mu_{\psi}\left(1-\phi_{\psi}\right)+\phi_{\psi} \psi_{t}\right)+C_{z \psi, n-1}\left(\mu_{z}\left(1-\phi_{z}\right)+\phi_{z} z_{t}\right)\right) \varepsilon_{\psi, t+1}
\end{array}\right]
$$

so that

$$
\begin{aligned}
& \operatorname{Var}_{t}\left(r_{n, t+1}\right)=\left[\begin{array}{c}
B_{x, n-1}^{2} \sigma_{X}^{2}+2 C_{z, n-1}^{2} 2 \sigma_{z}^{4}+2 C_{\psi, n-1}^{2} \sigma_{\psi}^{4}+C_{z \psi, n-1}^{2}\left(\sigma_{z}^{2} \sigma_{\psi}^{2}+\sigma_{z \psi}^{2}\right)+\left(B_{z, n-1}+2 C_{z, n-1} \mu_{z}\left(1-\phi_{z}\right)+C_{z \psi, n-1} \mu_{\psi}\left(1-\phi_{\psi}\right)\right)^{2} \sigma_{z}^{2} \\
+\left(B_{\psi, n-1}+2 C_{\psi, n-1} \mu_{\psi}\left(1-\phi_{\psi}\right)+C_{z \psi, n-1} \mu_{z}\left(1-\phi_{z}\right)\right)^{2} \sigma_{\psi}^{2} \\
+2\left(B_{z, n-1}+2 C_{z, n-1} \mu_{z}\left(1-\phi_{z}\right)+C_{z \psi, n-1} \mu_{\psi}\left(1-\phi_{\psi}\right)\right) \times\left(B_{\psi, n-1}+2 C_{\psi, n-1} \mu_{\psi}\left(1-\phi_{\psi}\right)+C_{z \psi, n-1} \mu_{z}\left(1-\phi_{z}\right)\right) \sigma_{z, \psi}
\end{array}\right] \\
& +\left[\begin{array}{c}
4\left(B_{z, n-1}+2 C_{z, n-1} \mu_{z}\left(1-\phi_{z}\right)+C_{z \psi, n-1} \mu_{\psi}\left(1-\phi_{\psi}\right)\right) C_{z, n-1} \phi_{z} \sigma_{z}^{2} \\
+2\left(B_{\psi, n-1}+2 C_{\psi, n-1} \mu_{\psi}\left(1-\phi_{\psi}\right)+C_{z \psi, n-1} \mu_{z}\left(1-\phi_{z}\right)\right) C_{z \psi, n-1} \phi_{z} \sigma_{\psi}^{2} \\
+2\left[\begin{array}{c}
2 C_{z, n-1}\left(B_{\psi, n-1}+2 C_{\psi, n-1} \mu_{\psi}\left(1-\phi_{\psi}\right)+C_{z \psi, n-1} \mu_{z}\left(1-\phi_{z}\right)\right) \\
+C_{z \psi, n-1}\left(B_{z, n-1}+2 C_{z, n-1} \mu_{z}\left(1-\phi_{z}\right)+C_{z \psi, n-1} \mu_{\psi}\left(1-\phi_{\psi}\right)\right)
\end{array}\right] \phi_{z} \sigma_{z, \psi}
\end{array}\right] z_{t} \\
& +\left[\begin{array}{c}
2\left(B_{z, n-1}+2 C_{z, n-1} \mu_{z}\left(1-\phi_{z}\right)+C_{z \psi, n-1} \mu_{\psi}\left(1-\phi_{\psi}\right)\right) C_{z \psi, n-1} \phi_{\psi} \sigma_{z}^{2} \\
+4\left(B_{\psi, n-1}+2 C_{\psi, n-1} \mu_{\psi}\left(1-\phi_{\psi}\right)+C_{z \psi, n-1} \mu_{z}\left(1-\phi_{z}\right)\right) C_{\psi, n-1} \phi_{\psi} \sigma_{\psi}^{2} \\
+2\left(B_{z, n-1}+2 C_{z, n-1} \mu_{z}\left(1-\phi_{z}\right)+C_{z \psi, n-1} \mu_{\psi}\left(1-\phi_{\psi}\right)\right) B_{x, n-1} \sigma_{x z} \\
+2\left(B_{\psi, n-1}+2 C_{\psi, n-1} \mu_{\psi}\left(1-\phi_{\psi}\right)+C_{z \psi, n-1} \mu_{z}\left(1-\phi_{z}\right)\right) B_{x, n-1} \sigma_{x \psi} \\
+2\left[\begin{array}{c}
2 C_{\psi, n-1}\left(B_{z, n-1}+2 C_{z, n-1} \mu_{z}\left(1-\phi_{z}\right)+C_{z \psi, n-1} \mu_{\psi}\left(1-\phi_{\psi}\right)\right) \\
+C_{z \psi, n-1}\left(B_{\psi, n-1}+2 C_{\psi, n-1} \mu_{\psi}\left(1-\phi_{\psi}\right)+C_{z \psi, n-1} \mu_{z}\left(1-\phi_{z}\right)\right)
\end{array}\right] \phi_{\psi} \sigma_{z, \psi}
\end{array}\right] \psi_{t} \\
& +\left[4 C_{z, n-1}^{2} \phi_{z}^{2} \sigma_{z}^{2}+C_{z \psi, n-1}^{2} \phi_{z}^{2} \sigma_{\psi}^{2}+4 C_{z, n-1} C_{z \psi, n-1} \phi_{z}^{2} \sigma_{z, \psi}\right] z_{t}^{2} \\
& +\left[B_{x, n-1}^{2} \sigma_{x}^{2}+C_{z \psi, n-1}^{2} \phi_{\psi}^{2} \sigma_{z}^{2}+4 C_{\psi, n-1}^{2} \phi_{\psi}^{2} \sigma_{\psi}^{2}+2 C_{z \psi, n-1} \phi_{\psi} B_{x, n-1} \sigma_{x z}+4 C_{\psi, n-1} \phi_{\psi} B_{x, n-1} \sigma_{x \psi}+4 C_{\psi, n-1} C_{z \psi, n-1} \phi_{\psi}^{2} \sigma_{z, \psi}\right] \psi_{t}^{2} \\
& +\left[\begin{array}{c}
4 C_{z, n-1} C_{z \psi, n-1} \phi_{z} \phi_{\psi} \sigma_{z}^{2}+4 C_{\psi, n-1} \phi_{\psi} C_{z \psi, n-1} \phi_{z} \phi_{\psi} \sigma_{\psi}^{2}+4 C_{z, n-1} \phi_{z} B_{x, n-1} \sigma_{x z} \\
+2 C_{z \psi, n-1} \phi_{z} B_{x, n-1} \sigma_{x \psi}+2\left(4 C_{z, n-1} C_{\psi, n-1}+C_{z \psi, n-1}^{2}\right) \sigma_{z \psi} \phi_{\psi} \phi_{z}
\end{array}\right] z_{t} \psi_{t}
\end{aligned}
$$


Volatility of Nominal Bond Returns We have

$$
r_{n, t+1}^{\$}-E_{t} r_{n, t+1}^{\$}=\left[\begin{array}{r}
B_{x, n-1}^{\$} \psi_{t} \varepsilon_{x, t+1}+B_{\$, n-1}^{\$} \varepsilon_{X, t+1}+B_{\lambda, n-1}^{\$} \psi_{t} \varepsilon_{\lambda, t+1}+B_{\lambda, n-1}^{\$} \varepsilon_{\Lambda, t+1}+B_{\xi, n-1}^{\$} \psi_{t} \varepsilon_{\xi, t+1} \\
+C_{z, n-1}^{\$} \varepsilon_{z, t+1}^{2}+C_{\psi, n-1}^{\$} \varepsilon_{\psi, t+1}^{2}+C_{z \psi, n-1}^{\$} \varepsilon_{z, t+1} \varepsilon_{\psi, t+1} \\
+\left(B_{z, n-1}^{\$}+2 C_{z, n-1}^{\$}\left(\mu_{z}\left(1-\phi_{z}\right)+\phi_{z} z_{t}\right)+C_{z \psi, n-1}^{\$}\left(\mu_{\psi}\left(1-\phi_{\psi}\right)+\phi_{\psi} \psi_{t}\right)\right) \varepsilon_{z, t+1} \\
+\left(B_{\psi, n-1}^{\$}+2 C_{\psi, n-1}^{\Phi}\left(\mu_{\psi}\left(1-\phi_{\psi}\right)+\phi_{\psi} \psi_{t}\right)+C_{z \psi, n-1}^{\$}\left(\mu_{z}\left(1-\phi_{z}\right)+\phi_{z} z_{t}\right)\right) \varepsilon_{\psi, t+1}
\end{array}\right]
$$


so that

$$
\begin{aligned}
& \operatorname{Var}_{t}\left(r_{n, t+1}^{\$}\right)=\left[\begin{array}{c}
B_{x, n-1}^{\$ 2} \sigma_{X}^{2}+B_{\lambda, n-1}^{\$ 2} \sigma_{\Lambda}^{2}+2 C_{z, n-1}^{\$ 2} \sigma_{z}^{4}+2 C_{\psi, n-1}^{\$ 2} \sigma_{\psi}^{4}+C_{z \psi, n-1}^{\$ 2}\left(\sigma_{z}^{2} \sigma_{\psi}^{2}+\sigma_{z \psi}^{2}\right) \\
+\left(B_{z, n-1}^{\$}+2 C_{z, n-1}^{\$} \mu_{z}\left(1-\phi_{z}\right)+C_{z \psi, n-1}^{\$} \mu_{\psi}\left(1-\phi_{\psi}\right)\right)^{2} \sigma_{z}^{2} \\
+\left(B_{\psi, n-1}^{\$}+2 C_{\psi, n-1}^{\$} \mu_{\psi}\left(1-\phi_{\psi}\right)+C_{z \psi, n-1}^{\$} \mu_{z}\left(1-\phi_{z}\right)\right)^{2} \sigma_{\psi}^{2} \\
+2\left(B_{\psi, n-1}^{\$}+2 C_{\psi, n-1}^{\$} \mu_{\psi}\left(1-\phi_{\psi}\right)+C_{z \psi, n-1}^{\$} \mu_{z}\left(1-\phi_{z}\right)\right) B_{\lambda, n-1}^{\$} \sigma_{\psi, \Lambda} \\
+2\left(B_{z, n-1}^{\$}+2 C_{z, n-1}^{\$} \mu_{z}\left(1-\phi_{z}\right)+C_{z \psi, n-1}^{\$} \mu_{\psi}\left(1-\phi_{\psi}\right)\right)\left(B_{\psi, n-1}^{\$}+2 C_{\psi, n-1}^{\$} \mu_{\psi}\left(1-\phi_{\psi}\right)+C_{z \psi, n-1}^{\$} \mu_{z}\left(1-\phi_{z}\right)\right) \sigma_{z, \psi}
\end{array}\right] \\
& +\left[\begin{array}{c}
4\left(B_{z, n-1}^{\$}+2 C_{z, n-1}^{\Phi} \mu_{z}\left(1-\phi_{z}\right)+C_{z \psi, n-1}^{\$} \mu_{\psi}\left(1-\phi_{\psi}\right)\right) C_{z, n-1}^{\$} \sigma_{z}^{2} \phi_{z} \\
+2\left(B_{\psi, n-1}^{\$}+2 C_{\psi, n-1}^{\$} \mu_{\psi}\left(1-\phi_{\psi}\right)+C_{z \psi, n-1}^{\$} \mu_{z}\left(1-\phi_{z}\right)\right) C_{z \psi, n-1}^{\$} \sigma_{\psi}^{2} \phi_{z} \\
+2 C_{z \psi, n-1}^{\$} B_{\lambda, n-1}^{\$} \sigma_{\psi, \Lambda} \phi_{z} \\
+2\left[\begin{array}{c}
2 C_{z, n-1}^{\$}\left(B_{\psi, n-1}^{\$}+2 C_{\psi, n-1}^{\$} \mu_{\psi}\left(1-\phi_{\psi}\right)+C_{z \psi, n-1}^{\$} \mu_{z}\left(1-\phi_{z}\right)\right) \\
+C_{z \psi, n-1}^{\$}\left(B_{z, n-1}^{\$}+2 C_{z, n-1}^{\$} \mu_{z}\left(1-\phi_{z}\right)+C_{z \psi, n-1}^{\$} \mu_{\psi}\left(1-\phi_{\psi}\right)\right)
\end{array}\right] \sigma_{z, \psi} \phi_{z}
\end{array}\right] z_{t} \\
& {\left[\begin{array}{rl} 
& 2\left(B_{z, n-1}^{\$}+2 C_{z, n-1}^{\$} \mu_{z}\left(1-\phi_{z}\right)+C_{z \psi, n-1}^{\$} \mu_{\psi}\left(1-\phi_{\psi}\right)\right) B_{x, n-1}^{\$} \sigma_{x z} \\
+ & 2\left(B_{\psi, n-1}^{\$}+2 C_{\psi, n-1}^{\$} \mu_{\psi}\left(1-\phi_{\psi}\right)+C_{z \psi, n-1}^{\$} \mu_{z}\left(1-\phi_{z}\right)\right) B_{x, n-1}^{\$} \sigma_{x \psi} \\
+ & 2\left(B_{z, n-1}^{\$}+2 C_{z, n-1}^{\$} \mu_{z}\left(1-\phi_{z}\right)+C_{z \psi, n-1}^{\$} \mu_{\psi}\left(1-\phi_{\psi}\right)\right) C_{z \psi, n-1}^{\$} \sigma_{z}^{2} \phi_{\psi}
\end{array}\right.} \\
& +4\left(B_{\psi, n-1}^{\$}+2 C_{\psi, n-1}^{\$} \mu_{\psi}\left(1-\phi_{\psi}\right)+C_{z \psi, n-1}^{\$} \mu_{z}\left(1-\phi_{z}\right)\right) C_{\psi, n-1}^{\$} \sigma_{\psi}^{2} \phi_{\psi} \\
& +2 B_{\lambda, n-1}^{\$ 2} \sigma_{\lambda, \Lambda} \\
& +2\left(B_{z, n-1}^{\$}+2 C_{z, n-1}^{\$} \mu_{z}\left(1-\phi_{z}\right)+C_{z \psi, n-1}^{\$} \mu_{\psi}\left(1-\phi_{\psi}\right)\right) B_{\lambda, n-1}^{\$} \sigma_{z, \lambda} \\
& +2\left(B_{\psi, n-1}^{\$}+2 C_{\psi, n-1}^{\$} \mu_{\psi}\left(1-\phi_{\psi}\right)+C_{z \psi, n-1}^{\Phi} \mu_{z}\left(1-\phi_{z}\right)\right) B_{\lambda, n-1}^{\Phi} \sigma_{\psi, \lambda} \\
& +2 B_{\lambda, n-1}^{\$} B_{\xi, n-1}^{\$} \sigma_{\Lambda, \xi}+4 C_{\psi, n-1}^{\$} B_{\lambda, n-1}^{\$} \sigma_{\psi, \Lambda} \phi_{\psi} \\
& +2\left(B_{z, n-1}^{\$}+2 C_{z, n-1}^{\$} \mu_{z}\left(1-\phi_{z}\right)+C_{z \psi, n-1}^{\Phi} \mu_{\psi}\left(1-\phi_{\psi}\right)\right) B_{\xi, n-1}^{\$} \sigma_{\xi, z} \\
& +2\left(B_{\psi, n-1}^{\$}+2 C_{\psi, n-1}^{\$} \mu_{\psi}\left(1-\phi_{\psi}\right)+C_{z \psi, n-1}^{\$} \mu_{z}\left(1-\phi_{z}\right)\right) B_{\xi, n-1}^{\$} \sigma_{\psi, \xi} \\
& \left.+2\left[\begin{array}{c}
2 C_{\psi, n-1}^{\$}\left(B_{z, n-1}^{\Phi}+2 C_{z, n-1}^{\Phi} \mu_{z}\left(1-\phi_{z}\right)+C_{z \psi, n-1}^{\$} \mu_{\psi}\left(1-\phi_{\psi}\right)\right) \\
+C_{z \psi, n-1}^{\Phi}\left(B_{\psi, n-1}^{\$}+2 C_{\psi, n-1}^{\$} \mu_{\psi}\left(1-\phi_{\psi}\right)+C_{z \psi, n-1}^{\Phi} \mu_{z}\left(1-\phi_{z}\right)\right)
\end{array}\right] \sigma_{z, \psi} \phi_{\psi}\right]
\end{aligned}
$$


$+\left[4 C_{z, n-1}^{\$ 2} \phi_{z}^{2} \sigma_{z}^{2}+C_{z \psi, n-1}^{\$ 2} \phi_{z}^{2} \sigma_{\psi}^{2}+4 C_{z, n-1}^{\$} C_{z \psi, n-1}^{\$} \sigma_{z, \psi} \phi_{z}^{2}\right] z_{t}^{2}$

$+\left[\begin{array}{c}B_{x, n-1}^{\$ 2} \sigma_{x}^{2}+B_{\lambda, n-1}^{\$ 2} \sigma_{\lambda}^{2}+B_{\xi, n-1}^{\$ 2} \sigma_{\xi}^{2}+2 B_{x, n-1}^{\$} B_{\lambda, n-1}^{\$} \sigma_{x, \lambda}+2 B_{x, n-1}^{\$} B_{\xi, n-1}^{\$} \sigma_{x, \xi} \\ +2 C_{z \psi, n-1}^{\$} B_{x, n-1}^{\$} \sigma_{x z} \phi_{\psi}+4 C_{\psi, n-1}^{\$} B_{x, n-1}^{\$} \sigma_{x \psi} \phi_{\psi}+C_{z \psi, n-1}^{\$ 2} \phi_{\psi}^{2} \sigma_{z}^{2}+4 C_{\psi, n-1}^{\$} \phi_{\psi}^{2} \sigma_{\psi}^{2}+2 B_{\lambda, n-1}^{\$} B_{\xi, n-1}^{\$} \sigma_{\xi \lambda}+2 C_{z \psi, n-1}^{\$} B_{\lambda, n-1}^{\$} \sigma_{z, \lambda} \phi_{\psi} \\ +4 C_{\psi, n-1}^{\$} B_{\lambda, n-1}^{\$} \sigma_{\psi, \lambda} \phi_{\psi}+2 C_{z \psi, n-1}^{\$} B_{\xi, n-1}^{\$} \sigma_{\xi, z} \phi_{\psi}+4 C_{\psi, n-1}^{\$} B_{\xi, n-1}^{\$} \sigma_{\psi, \xi} \phi_{\psi}+4 C_{\psi, n-1}^{\$} C_{z \psi, n-1}^{\$} \sigma_{z, \psi} \phi_{\psi}^{2}\end{array}\right] \psi_{t}^{2}$

$+\left[\begin{array}{c}4 C_{z, n-1}^{\$} B_{x, n-1}^{\$} \sigma_{x z} \phi_{z}+2 C_{z \psi, n-1}^{\$} B_{x, n-1}^{\$} \sigma_{x \psi} \phi_{z}+4 C_{z, n-1}^{\$} C_{z \psi, n-1}^{\$} \sigma_{z}^{2} \phi_{z} \phi_{\psi} \\ +4 C_{\psi, n-1}^{\$} C_{z \psi, n-1}^{\$} \sigma_{\psi}^{2} \phi_{z} \phi_{\psi}+4 C_{z, n-1}^{\Phi} B_{\lambda, n-1}^{\$} \sigma_{z, \lambda} \phi_{z}+2 C_{z \psi, n-1}^{\Phi} B_{\lambda, n-1}^{\$} \sigma_{\psi, \lambda} \phi_{z} \\ +4 C_{z, n-1}^{\$} B_{\xi, n-1}^{\$} \sigma_{\xi, z} \phi_{z}+2 C_{z \psi, n-1}^{\$} B_{\xi, n-1}^{\$} \sigma_{\psi, \xi} \phi_{z}+2\left(4 C_{z, n-1}^{\$} C_{\psi, n-1}^{\$}+C_{z \psi, n-1}^{\$ 2}\right) \sigma_{z \psi} \phi_{\psi} \phi_{z}\end{array}\right] z_{t} \psi_{t}$ 


\section{A.3 Derivations for Constant- $z$ Model}

\section{A.3.1 State Variables Processes}

The state variables in the constant- $z$ version of the model follow the processes:

$$
\begin{aligned}
-m_{t+1} & =x_{t}+\frac{1}{2} \sigma_{m}^{2}+\varepsilon_{m, t+1} \\
x_{t+1} & =\mu_{x}\left(1-\phi_{x}\right)+\phi_{x} x_{t}+\psi_{t} \varepsilon_{x, t+1}+\varepsilon_{X, t+1}
\end{aligned}
$$

$$
\begin{aligned}
\pi_{t+1} & =\lambda_{t}+\xi_{t}+\frac{1}{2} \psi_{t}^{2} \sigma_{\pi}^{2}+\psi_{t} \varepsilon_{\pi, t+1} \\
\lambda_{t+1} & =\lambda_{t}+\psi_{t} \varepsilon_{\lambda, t+1}+\varepsilon_{\Lambda, t+1} \\
\xi_{t+1} & =\phi_{\xi} \xi_{t}+\psi_{t} \varepsilon_{\xi, t+1} \\
\psi_{t+1} & =\mu_{\psi}\left(1-\phi_{\psi}\right)+\phi_{\psi} \psi_{t}+\varepsilon_{\psi, t+1}
\end{aligned}
$$

\section{A.3.2 Pricing Equations}

Real Term Structure The price of a single-period zero-coupon real bond satisfies

$$
P_{1, t}=E_{t}\left[\exp \left\{m_{t+1}\right\}\right]=-x_{t}-\frac{1}{2} \sigma_{m}^{2}+\frac{1}{2} \sigma_{m}^{2}=-x_{t}
$$

1. We conjecture that the price function is exponential affine in $x_{t}$ and $z_{t}$ with the form

$$
P_{n, t}=\exp \left\{A_{n}+B_{x, n} x_{t}+B_{\psi, n} \psi_{t}+C_{\psi, n} \psi_{t}^{2}\right\} .
$$

The standard pricing equation implies

$$
\begin{aligned}
P_{n, t}= & E_{t}\left[\exp \left\{p_{n-1, t+1}+m_{t+1}\right\}\right]=E_{t}\left[\exp \left\{A_{n-1}+B_{x, n-1} x_{t+1}+B_{\psi, n-1} \psi_{t+1}+C_{\psi, n-1} \psi_{t+1}^{2} \cdot x_{t}-\frac{1}{2} \sigma_{m}^{2}-\varepsilon_{m, t+1}\right\}\right] \\
= & E_{t}\left[\exp \left\{\begin{array}{r}
A_{n-1}+B_{x, n-1}\left(\left(1-\phi_{x}\right) \mu_{x}+\phi_{x} x_{t}+\psi_{t} \varepsilon_{x, t+1}+\varepsilon_{X, t+1}\right)+B_{\psi, n-1}\left(\left(1-\phi_{\psi}\right) \mu_{\psi}+\phi_{\psi} \psi_{t}+\varepsilon_{\psi, t+1}\right) \\
+C_{\psi, n-1}\left(\left(1-\phi_{\psi}\right) \mu_{\psi}+\phi_{\psi} \psi_{t}+\varepsilon_{\psi, t+1}\right)^{2} \cdot-x_{t}-\frac{1}{2} \sigma_{m}^{2}-\varepsilon_{m, t+1}
\end{array}\right]\right. \\
= & A_{n-1}+B_{x, n-1}\left(\left(1-\phi_{x}\right) \mu_{x}+\phi_{x} x_{t}\right)+B_{\psi, n-1}\left(\left(1-\phi_{\psi}\right) \mu_{\psi}+\phi_{\psi} \psi_{t}\right)+C_{\psi, n-1}\left(\mu_{\psi}\left(1-\phi_{\psi}\right)+\phi_{\psi} \psi_{t}\right)^{2}-x_{t}-\frac{1}{2} \sigma_{m}^{2} \\
& \times E_{t}\left[\exp \left\{\mathbf{d}_{1}^{\prime} \boldsymbol{\omega}_{t+1}+\boldsymbol{\omega}_{t+1}^{\prime} \mathbf{D}_{2} \boldsymbol{\omega}_{t+1}\right\}\right]
\end{aligned}
$$


where $\boldsymbol{\omega}_{t+1}^{\prime}=\left(\varepsilon_{X, t+1}, \varepsilon_{m, t+1}, \varepsilon_{x, t+1}, \varepsilon_{\psi, t+1}\right)^{\sim} N\left(0, \boldsymbol{\Sigma}_{\omega}\right)$,

$$
\begin{gathered}
\mathbf{d}_{1}=\left(\begin{array}{c}
B_{x, n-1} \\
-1 \\
B_{x, n-1} \psi_{t} \\
B_{\psi, n-1}+2 C_{\psi, n-1}\left(\mu_{\psi}\left(1-\phi_{\psi}\right)+\phi_{\psi} \psi_{t}\right)
\end{array}\right) \\
\mathbf{D}_{2}=\left(\begin{array}{cc}
0 & 0 \\
0 & \\
0 & C_{\psi, n-1}
\end{array}\right)
\end{gathered}
$$

Following Campbell, Chan, and Viceira (2003), we complete the square to calculate

$$
\begin{aligned}
E_{t}\left[\exp \left\{\mathbf{d}_{1}^{\prime} \boldsymbol{\omega}_{t+1}+\boldsymbol{\omega}_{t+1}^{\prime} \mathbf{D}_{2} \boldsymbol{\omega}_{t+1}\right\}\right] & =\frac{\left|\boldsymbol{\Sigma}_{\omega}\right|^{-1 / 2}}{\left|\boldsymbol{\Sigma}_{\omega}^{-1}-2 \mathbf{D}_{2}\right|^{1 / 2}} \exp \left\{\frac{1}{2} \mathbf{d}_{1}\left(\boldsymbol{\Sigma}_{\omega}^{-1}-2 \mathbf{D}_{2}\right)^{-1} \mathbf{d}_{1}^{\prime}\right\} \\
& =\exp \left\{-\frac{1}{2} \log \left|\boldsymbol{\Sigma}_{\omega}\right|+\frac{1}{2} \log |\mathbf{G}|+\frac{1}{2} \mathbf{d}_{1} \mathbf{G} \mathbf{d}_{1}^{\prime}\right\}
\end{aligned}
$$

where $\mathbf{G}=\left(\boldsymbol{\Sigma}_{\omega}^{-1}-2 \mathbf{D}_{2}\right)^{-1}$. Let $g_{i j}$ be the $i j$-th element of $\mathbf{G}$. Then expanding and collecting terms gives

$$
\begin{aligned}
& p_{n, t}= {\left[\begin{array}{c}
A_{n-1}+B_{x, n-1}\left(\left(1-\phi_{x}\right) \mu_{x}+\phi_{x} x_{t}\right)+B_{\psi, n-1}\left(\left(1-\phi_{\psi}\right) \mu_{\psi}+\phi_{\psi} \psi_{t}\right)+C_{\psi, n-1}\left(\mu_{\psi}\left(1-\phi_{\psi}\right)+\phi_{\psi} \psi_{t}\right)^{2} \\
-x_{t}-\frac{1}{2} \sigma_{m}^{2}-\frac{1}{2} \log \left|\boldsymbol{\Sigma}_{\omega}\right|+\frac{1}{2} \log |\mathbf{G}|+\frac{1}{2} g_{11} B_{x, n-1}^{2}+\frac{1}{2} g_{22}+\frac{1}{2} g_{33} B_{x, n-1}^{2} \psi_{t}^{2} \\
+\frac{1}{2} g_{44}\left(B_{\psi, n-1}+2 C_{\psi, n-1}\left(\mu_{\psi}\left(1-\phi_{\psi}\right)+\phi_{\psi} \psi_{t}\right)\right)^{2} \\
-g_{12} B_{x, n-1}+g_{13} B_{x, n-1}^{2} \psi_{t}+g_{14} B_{x, n-1}\left(B_{\psi, n-1}+2 C_{\psi, n-1}\left(\mu_{\psi}\left(1-\phi_{\psi}\right)+\phi_{\psi} \psi_{t}\right)\right) \\
-g_{23} B_{x, n-1} \psi_{t}-g_{24}\left(B_{\psi, n-1}+2 C_{\psi, n-1}\left(\mu_{\psi}\left(1-\phi_{\psi}\right)+\phi_{\psi} \psi_{t}\right)\right) \\
+g_{34} B_{x, n-1} \psi_{t}\left(B_{\psi, n-1}+2 C_{\psi, n-1}\left(\mu_{\psi}\left(1-\phi_{\psi}\right)+\phi_{\psi} \psi_{t}\right)\right) \\
A_{n-1}+B_{x, n-1}\left(1-\phi_{x}\right) \mu_{x}+B_{x, n-1} \phi_{x} x_{t}+B_{\psi, n-1}\left(1-\phi_{\psi}\right) \mu_{\psi}+B_{\psi, n-1} \phi_{\psi} \psi_{t} \\
+C_{\psi, n-1} \mu_{\psi}^{2}\left(1-\phi_{\psi}\right)^{2}+2 C_{\psi, n-1} \mu_{\psi}\left(1-\phi_{\psi}\right) \phi_{\psi} \psi_{t}+C_{\psi, n-1} \phi_{\psi}^{2} \psi_{t}^{2} \\
-x_{t}-\frac{1}{2} \sigma_{m}^{2}-\frac{1}{2} \log \left|\boldsymbol{\Sigma}_{\omega}\right|+\frac{1}{2} \log |\mathbf{G}|+\frac{1}{2} g_{11} B_{x, n-1}^{2}+\frac{1}{2} g_{22}+\frac{1}{2} g_{33} B_{x, n-1}^{2} \psi_{t}^{2} \\
=
\end{array}\right.} \\
& {\left[\begin{array}{c}
2 \\
+\frac{1}{2} g_{44}\left(B_{\psi, n-1}+2 C_{\psi, n-1} \mu_{\psi}\left(1-\phi_{\psi}\right)\right)^{2}+2 g_{44} C_{\psi, n-1}^{2} \phi_{\psi}^{2} \psi_{t}^{2}+2 g_{44}\left(B_{\psi, n-1}+2 C_{\psi, n-1} \mu_{\psi}\left(1-\phi_{\psi}\right)\right) C_{\psi, n-1} \phi_{\psi} \psi_{t} \\
-g_{12} B_{x, n-1}+g_{13} B_{x, n-1}^{2} \psi_{t}+g_{14} B_{x, n-1}\left(B_{\psi, n-1}+2 C_{\psi, n-1} \mu_{\psi}\left(1-\phi_{\psi}\right)\right)+2 g_{14} B_{x, n-1} C_{\psi, n-1} \phi_{\psi} \psi_{t} \\
-g_{23} B_{x, n-1} \psi_{t}-g_{24}\left(B_{\psi, n-1}+2 g_{24} C_{\psi, n-1} \mu_{\psi}\left(1-\phi_{\psi}\right)\right)-2 g_{24} C_{\psi, n-1} \phi_{\psi} \psi_{t} \\
+g_{34} B_{x, n-1} \psi_{t}\left(B_{\psi, n-1}+2 B_{x, n-1} C_{\psi, n-1} \mu_{\psi}\left(1-\phi_{\psi}\right)\right)+2 g_{34} B_{x, n-1} C_{\psi, n-1} \phi_{\psi} \psi_{t}^{2}
\end{array}\right] }
\end{aligned}
$$

Expanding and collecting terms yields 


$$
\begin{aligned}
A_{n}= & {\left[\begin{array}{c}
A_{n-1}+B_{x, n-1}\left(1-\phi_{x}\right) \mu_{x}+B_{\psi, n-1}\left(1-\phi_{\psi}\right) \mu_{\psi}+C_{\psi, n-1} \mu_{\psi}^{2}\left(1-\phi_{\psi}\right)^{2} \\
-\frac{1}{2} \sigma_{m}^{2}-\frac{1}{2} \log \left|\mathbf{\Sigma}_{\omega}\right|+\frac{1}{2} \log |\mathbf{G}|+\frac{1}{2} g_{11} B_{x, n-1}^{2}+\frac{1}{2} g_{22} \\
+\frac{1}{2} g_{44}\left(B_{\psi, n-1}+2 C_{\psi, n-1} \mu_{\psi}\left(1-\phi_{\psi}\right)\right)^{2}-g_{12} B_{x, n-1}+g_{14} B_{x, n-1}\left(B_{\psi, n-1}+2 C_{\psi, n-1} \mu_{\psi}\left(1-\phi_{\psi}\right)\right) \\
-g_{24}\left(B_{\psi, n-1}+2 g_{24} C_{\psi, n-1} \mu_{\psi}\left(1-\phi_{\psi}\right)\right)
\end{array}\right] } \\
B_{x, n}= & B_{x, n-1} \phi_{x}-1 \quad \\
B_{\psi, n}= & {\left[\begin{array}{c}
B_{\psi, n-1} \phi_{\psi}+2 C_{\psi, n-1} \mu_{\psi}\left(1-\phi_{\psi}\right) \phi_{\psi}+2 g_{44}\left(B_{\psi, n-1}+2 C_{\psi, n-1} \mu_{\psi}\left(1-\phi_{\psi}\right)\right) C_{\psi, n-1} \phi_{\psi} \\
+g_{13} B_{x, n-1}^{2}+2 g_{14} B_{x, n-1} C_{\psi, n-1} \phi_{\psi}-g_{23} B_{x, n-1}-2 g_{24} C_{\psi, n-1} \phi_{\psi} \\
+g_{34} B_{x, n-1}\left(B_{\psi, n-1}+2 C_{\psi, n-1} \mu_{\psi}\left(1-\phi_{\psi}\right)\right)
\end{array}\right] } \\
C_{\psi, n}= & {\left[\begin{array}{c}
C_{\psi, n-1} \phi_{\psi}^{2}+\frac{1}{2} g_{33} B_{x, n-1}^{2}+2 g_{44} C_{\psi, n-1}^{2} \phi_{\psi}^{2}+2 g 4 B_{x, n-1} C_{\psi, n-1} \phi_{\psi}
\end{array}\right] }
\end{aligned}
$$

Nominal Term Structure The price of a single-period zero-coupon nominal bond satisfies

$$
\begin{aligned}
P_{1, t}^{\Phi} & =E_{t}\left[\exp \left\{m_{t+1}-\pi_{t+1}\right\}\right] \\
& =E_{t}\left[\exp \left\{-x_{t}-\frac{1}{2} \sigma_{m}^{2}-\varepsilon_{m, t+1}-\lambda_{t}-\xi_{t}-\frac{1}{2} \psi_{t}^{2} \sigma_{\pi}^{2}-\psi_{t} \varepsilon_{\pi, t+1}\right\}\right] \\
& =\exp \left\{-x_{t}-\frac{1}{2} \sigma_{m}^{2}-\lambda_{t}-\xi_{t}-\frac{1}{2} \psi_{t}^{2} \sigma_{\pi}^{2}+\frac{1}{2} \sigma_{m}^{2}+\frac{1}{2} \psi_{t}^{2} \sigma_{\pi}^{2}+\psi_{t} \sigma_{m \pi}\right\} \\
& =\exp \left\{-x_{t}-\lambda_{t}-\xi_{t}+\psi_{t} \sigma_{m \pi}\right\}
\end{aligned}
$$

where the last equality follows from the joint conditional normality of $z_{t} \varepsilon_{m, t+1}$ and $\psi_{t} \varepsilon_{\pi, t+1}$.

We now guess that the price function is exponential linear-quadratic in the state variables with the following form:

$$
P_{n, t}^{\Phi}=\exp \left\{A_{n}^{\$}+B_{x, n}^{\$} x_{t}+B_{\lambda, n}^{\$} \lambda_{t}+B_{\xi, n}^{\$} \xi_{t}+B_{\psi, n}^{\$} \psi_{t}+C_{\psi, n}^{\$} \psi_{t}^{2}\right\}
$$

The standard pricing equation then implies

$$
\begin{aligned}
P_{n, t}^{\$}= & E_{t}\left[\exp \left\{p_{n-1, t+1}^{\$}+m_{t+1}-\pi_{t+1}\right\}\right] \\
= & E_{t}\left[\exp \left\{\begin{array}{c}
\left.A_{n-1}^{\$}+B_{x, n-1}^{\Phi} x_{t+1}+B_{\lambda, n-1}^{\$} \lambda_{t+1}+B_{\xi, n-1}^{\$} \xi_{t+1}+B_{\psi, n-1}^{\$} \psi_{t+1}+C_{\psi, n-1}^{\$} \psi_{t+1}^{2}\right\} \\
-x_{t}-\frac{1}{2} \sigma_{m}^{2}-\varepsilon_{m, t+1}-\lambda_{t}-\xi_{t}-\frac{1}{2} \psi_{t}^{2} \sigma_{\pi}^{2}-\psi_{t} \varepsilon_{\pi, t+1}
\end{array}\right]\right. \\
= & \exp \left\{\begin{array}{c}
A_{n-1}^{\$}+B_{x, n-1}^{\$}\left(\mu_{x}\left(1-\phi_{x}\right)+\phi_{x} x_{t}\right)+B_{\lambda, n-1}^{\$}\left(\mu_{\lambda}+\lambda_{t}\right)+B_{\xi, n-1}^{\$} \phi_{\xi} \xi_{t} \\
+B_{\psi, n-1}^{\$}\left(\mu_{\psi}\left(1-\phi_{\psi}\right)+\phi_{\psi} \psi_{t}\right)+C_{\psi, n-1}^{\Phi}\left(\mu_{\psi}\left(1-\phi_{\psi}\right)+\phi_{\psi} \psi_{t}\right)^{2} \\
-x_{t}-\frac{1}{2} \sigma_{m}^{2}-\lambda_{t}-\xi_{t}-\frac{1}{2} \psi_{t}^{2} \sigma_{\pi}^{2}
\end{array}\right\} \\
& \times E_{t}\left[\exp \left\{\mathbf{d}_{1}^{\$ \prime} \boldsymbol{\omega}_{t+1}^{\$}+\boldsymbol{\omega}_{t+1}^{\$ \prime} \mathbf{D}_{2}^{\$} \boldsymbol{\omega}_{t+1}^{\$}\right\}\right]
\end{aligned}
$$


where $\boldsymbol{\omega}_{t+1}^{\$ \prime}=\left(\varepsilon_{X, t+1}, \varepsilon_{\Lambda, t+1}, \varepsilon_{\lambda, t+1}, \varepsilon_{m, t+1}, \varepsilon_{\pi, t+1}, \varepsilon_{x, t+1}, \varepsilon_{\xi, t+1}, \varepsilon_{\psi, t+1}\right)^{\sim} N\left(0, \boldsymbol{\Sigma}_{\omega}^{\$}\right)$,

$$
\begin{aligned}
& \mathbf{d}_{1}^{\S}=\left(\begin{array}{c}
B_{x, n-1}^{\$} \\
B_{\lambda, n-1}^{\S} \\
B_{\lambda, n-1}^{\S} \psi_{t} \\
-1 \\
-\psi_{t} \\
B_{x, n-1}^{\S} \psi_{t} \\
B_{\xi, n-1}^{\S} \psi_{t} \\
B_{\psi, n-1}^{\S}+2 C_{\psi, n-1}^{\$}\left(\mu_{\psi}\left(1-\phi_{\psi}\right)+\phi_{\psi} \psi_{t}\right)
\end{array}\right) \\
& \mathbf{D}_{2}^{\$}=\left(\begin{array}{ccc}
0 & \cdots & 0 \\
& & \vdots \\
\vdots & \ddots & \\
0 & \cdots & C_{\psi, n-1}^{\$}
\end{array}\right)
\end{aligned}
$$

Following Campbell, Chan, and Viceira (2003), we complete the square to calculate

$$
\begin{aligned}
E_{t}\left[\exp \left\{\mathbf{d}_{1}^{\$ \prime} \boldsymbol{\omega}_{t+1}^{\$}+\boldsymbol{\omega}_{t+1}^{\$ \prime} \mathbf{D}_{2}^{\$} \boldsymbol{\omega}_{t+1}^{\S}\right\}\right] & =\frac{\left|\boldsymbol{\Sigma}_{\omega}^{\$}\right|^{-1 / 2}}{\left|\boldsymbol{\Sigma}_{\omega}^{\$-1}-2 \mathbf{D}_{2}^{\S}\right|^{1 / 2}} \exp \left\{\frac{1}{2} \mathbf{d}_{1}^{\S}\left(\boldsymbol{\Sigma}_{\omega}^{\$-1}-2 \mathbf{D}_{2}^{\$}\right)^{-1} \mathbf{d}_{1}^{\$ \prime}\right\} \\
& =\exp \left\{-\frac{1}{2} \log \left|\boldsymbol{\Sigma}_{\omega}^{\S}\right|+\frac{1}{2} \log \left|\mathbf{G}^{\$}\right|+\frac{1}{2} \mathbf{d}_{1}^{\$} \mathbf{G}^{\$} \mathbf{d}_{1}^{\$ \prime}\right\}
\end{aligned}
$$

where $\mathbf{G}^{\$}=\left(\boldsymbol{\Sigma}_{\omega}^{\$-1}-2 \mathbf{D}_{2}^{\$}\right)^{-1}$. Let $g_{i j}^{\$}$ be the $i j$-th element of $\mathbf{G}$. Then expanding and collecting terms gives $g^{\$}$ 


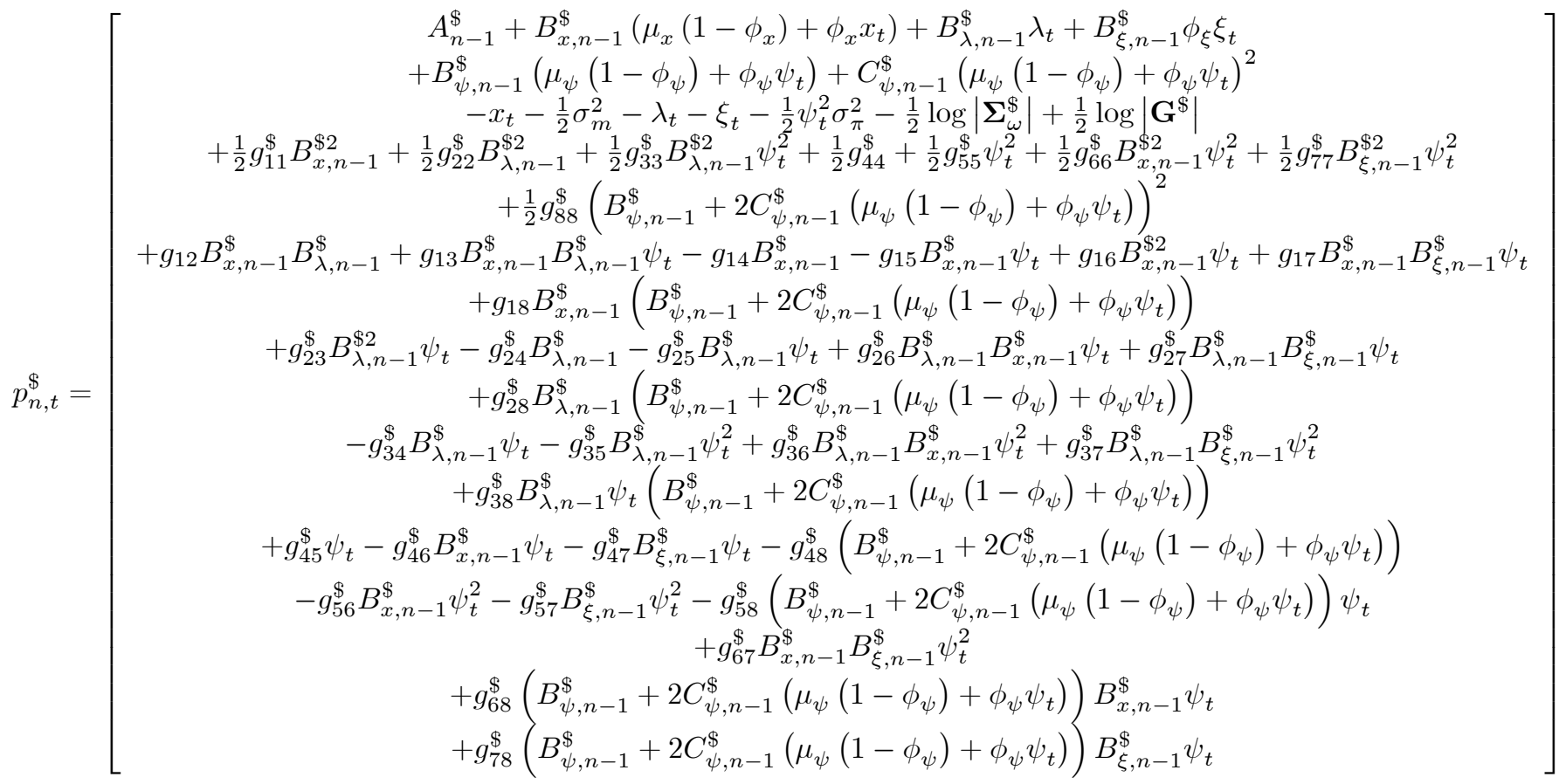

Thus, the coefficients of the pricing equation satisfy

$$
\begin{aligned}
& A_{n}^{\$}=\left[\begin{array}{c}
A_{n-1}^{\$}+B_{x, n-1}^{\$} \mu_{x}\left(1-\phi_{x}\right)+B_{\psi, n-1}^{\$} \mu_{\psi}\left(1-\phi_{\psi}\right)+C_{\psi, n-1}^{\$} \mu_{\psi}^{2}\left(1-\phi_{\psi}\right)^{2}-\frac{1}{2} \sigma_{m}^{2}-\frac{1}{2} \log \left|\mathbf{\Sigma}_{\omega}^{\$}\right|+\frac{1}{2} \log \left|\mathbf{G}^{\$}\right| \\
+\frac{1}{2} g_{11}^{\$} B_{x, n-1}^{\$ 2}+\frac{1}{2} g_{22}^{\$} B_{\lambda, n-1}^{\$ 2}+\frac{1}{2} g_{44}^{\$}+\frac{1}{2} g_{88}^{\$}\left(B_{\psi, n-1}^{\$}+2 C_{\psi, n-1}^{\$} \mu_{\psi}\left(1-\phi_{\psi}\right)\right)^{2} \\
+g_{12} B_{x, n-1}^{\$} B_{\lambda, n-1}^{\$}-g_{14} B_{x, n-1}^{\$}+g_{18} B_{x, n-1}^{\$}\left(B_{\psi, n-1}^{\$}+2 C_{\psi, n-1}^{\$} \mu_{\psi}\left(1-\phi_{\psi}\right)\right) \\
-g_{24}^{\$} B_{\lambda, n-1}^{\$}+g_{28}^{\$} B_{\lambda, n-1}^{\$}\left(B_{\psi, n-1}^{\$}+2 C_{\psi, n-1}^{\$} \mu_{\psi}\left(1-\phi_{\psi}\right)\right)-g_{48}^{\$}\left(B_{\psi, n-1}^{\$}+2 C_{\psi, n-1}^{\$} \mu_{\psi}\left(1-\phi_{\psi}\right)\right)
\end{array}\right] \\
& B_{x, n}^{\$}=B_{x, n-1}^{\$} \phi_{x}-1 \\
& B_{\lambda, n}^{\$}=B_{\lambda, n-1}^{\$}-1 \\
& B_{\xi, n}^{\$}=B_{\xi, n-1}^{\$} \phi_{\xi}-1
\end{aligned}
$$




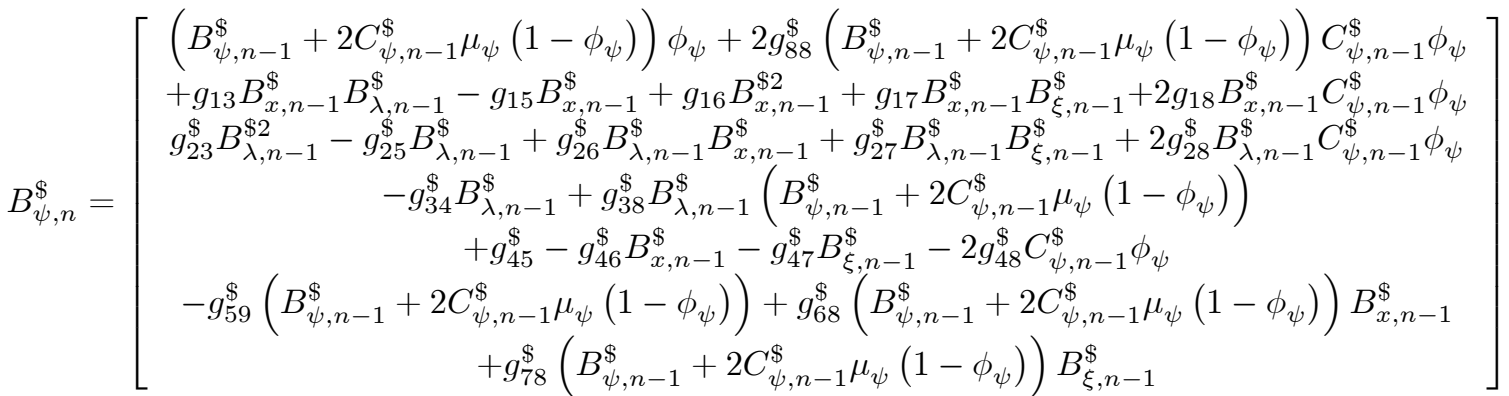

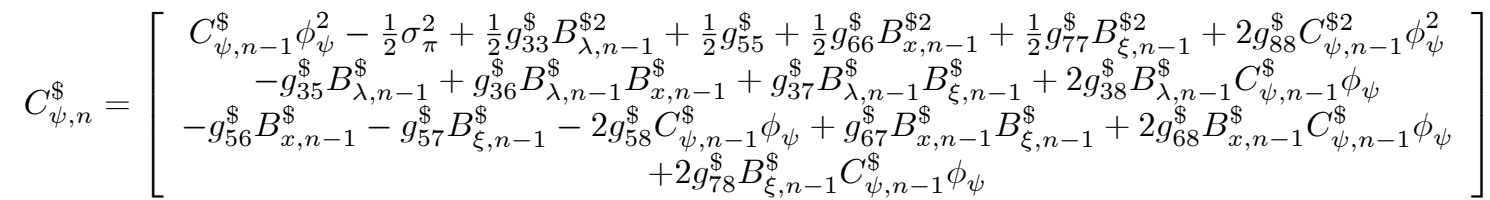

where $B_{x, 1}^{\$}=-1, B_{\lambda, 1}^{\$}=-1, B_{\xi, 1}^{\$}=-1, B_{\psi, 1}^{\$}=\sigma_{m \pi}$ and all other coefficients are zero at $n=1$. 